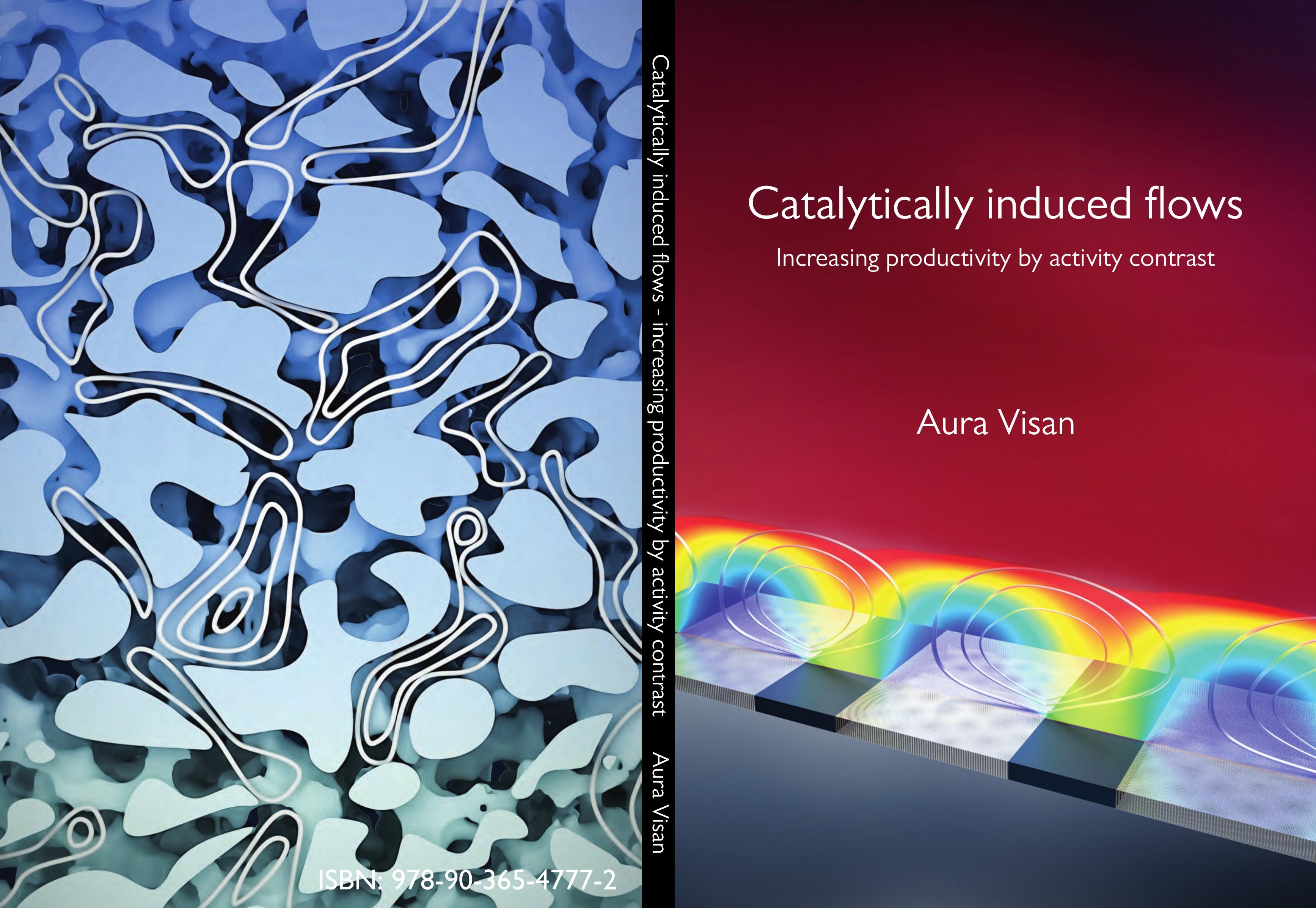


Catalytically induced flows - increasing productivity by activity contrast

Aura Visan 


\section{Graduation committee:}

Prof. dr. J. L. Herek (chairman)

Prof.dr.ir. R. G. H. Lammertink (supervisor)

Prof. dr. ret. nat. D. Lohse

Prof. dr. J. Eijkel

Prof. L. Joly

Prof. dr.ir. K. Schroen

Prof. dr. R. Tuinier
University of Twente

University of Twente

University of Twente

University of Twente

Université Lyon 1

Wageningen University

Eindhoven University of Technology

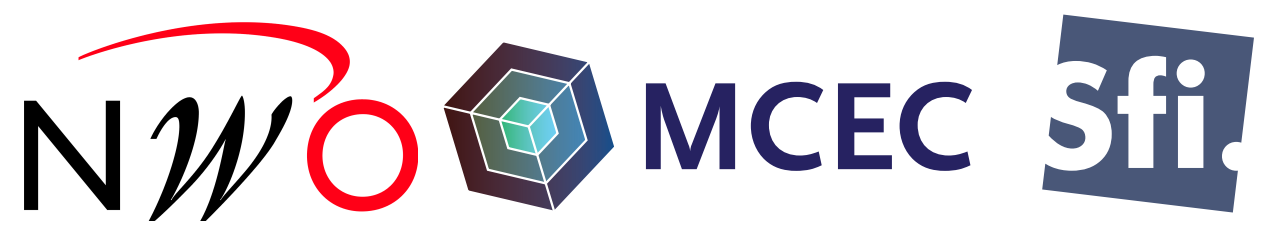

The work in this thesis was carried out at the Soft matter, fluidics and interfaces group of the Faculty of Science and Technology of the University of Twente. This work was supported by the Netherlands Center for Multiscale Catalytic Energy Conversion (MCEC), an NWO Gravitation programme funded by the Ministry of Education, Culture and Science of the government of the Netherlands.

Publisher:

Aura Visan, Soft matter, fluidics and interfaces, University of Twente, P.O. Box 217, 7500 AE Enschede, The Netherlands

Copyright @ 92019 by Aura Visan. All rights reserved.

No part of this work may be reproduced or transmitted for commercial purposes, in any form or by any means, electronic or mechanical, including photocopying and recording, or by any information storage or retrieval system, except as expressly permitted by the publisher.

ISBN: 978-90-365-4777-2

DOI: $10.3990 / 1.9789036547772$

URL: https://doi.org/10.3990/1.9789036547772 


\title{
CATAlyticAlLy INDUCED FLOWS - INCREASING PRODUCTIVITY BY ACTIVITY CONTRAST
}

\author{
DISSERTATION
}

to obtain

the degree of doctor at the University of Twente, on the authority of the rector magnificus, prof.dr. T.T.M. Palstra,

on account of the decision of the graduation committee, to be publicly defended

on Friday, May $24^{\text {th }}, 2019$ at 12:45 hrs

by

Aura Visan

Born on September $21^{\text {st }}, 1987$

in Tulcea, Romania 
This dissertation has been approved by the promotor:

\author{
prof.dr.ir. R. G. H. Lammertink
}


Science is a bit like the joke about the drunk who is looking under a lamppost for a key that he has lost on the other side of the street, because that's where the light is. He has no other choice.

Noam Chomsky 



\section{Contents}

1 Introduction 1

1.1 Mass transport limitations in heterogeneous catalysis . . . . . . 1

1.2 Historical background on diffusio-osmosis . . . . . . . . . . 3

1.3 Applications and recent developments . . . . . . . . . . 6

1.4 Diffusio-osmosis induced by catalytic surfaces . . . . . . . . 8

1.5 Scope of the thesis . . . . . . . . . . . . . . 10

2 Photocatalytic reactor design: guidelines for kinetic investigation 15

2.1 Introduction . . . . . . . . . . . . . . . . 17

2.2 Electron-hole pair generation . . . . . . . . . . . . 18

2.3 Radiative transfer . . . . . . . . . . . . . . . . . . 21

2.4 Mass transport . . . . . . . . . . . . . . . . . . 28

2.5 Reactor design and operation . . . . . . . . . . . . 32

2.6 Conclusions . . . . . . . . . . . . . . . . . . 34

3 Modeling intrinsic kinetics in immobilized photocatalytic microreactors

3.1 Introduction . . . . . . . . . . . . . . . . . . . . 39

3.2 Materials and Methods . . . . . . . . . . . . . . . . . 42

3.3 Model . . . . . . . . . . . . . . . . . . . . . . . 47

3.4 Results and discussion . . . . . . . . . . . . . 50

3.5 Conclusions . . . . . . . . . . . . . . . . . 57

4 Fructose dehydration to hydroxyl-methylfurfural in an immobilized catalytic microreactor $\quad 59$

4.1 Introduction . . . . . . . . . . . . . . . . . 61

4.2 Experimental . . . . . . . . . . . . . . . . 63

4.3 Results and discussion . . . . . . . . . . . . . 68 
4.4 Conclusion . . . . . . . . . . . . . . . . . 74

5 Reaction driven diffusio-osmotic flow inside catalytic dead-end $\begin{array}{ll}\text { pores } & \mathbf{7 7}\end{array}$

5.1 Introduction . . . . . . . . . . . . . . . . . . 79

5.2 Experimental . . . . . . . . . . . . . . . . . 81

5.3 Model . . . . . . . . . . . . . . . . . . . . . 82

5.4 Results and discussion . . . . . . . . . . . . . 86

5.5 Conclusion . . . . . . . . . . . . . . . . . 91

6 Catalytically induced flows: Increasing conversion with less $\begin{array}{ll}\text { catalyst } & 93\end{array}$

6.1 Introduction . . . . . . . . . . . . . . . . . . 95

6.2 Model . . . . . . . . . . . . . . . . . . . . . . . . 97 97

6.3 Results and discussion . . . . . . . . . . . . . . . . 100

6.4 Conclusion . . . . . . . . . . . . . . . . 108

7 Reaction induced diffusio-phoresis of ordinary catalytic par$\begin{array}{ll}\text { ticles } & 109\end{array}$ 7.1 Introduction . . . . . . . . . . . . . . . . . 111

7.2 Methods . . . . . . . . . . . . . . . . . . . 114

7.3 Results and discussion . . . . . . . . . . . . . . . 118

7.4 Conclusions . . . . . . . . . . . . . . . . . 123

8 Summary and Outlook $\quad \mathbf{1 2 5}$

8.1 Summary . . . . . . . . . . . . . . . . . 125

8.2 Outlook . . . . . . . . . . . . . . 126

$\begin{array}{ll}\text { Samenvatting } & 133\end{array}$

$\begin{array}{ll}\text { Acknowledgements } & 135\end{array}$

$\begin{array}{ll}\text { References } & 137\end{array}$ 


\section{Introduction}

\subsection{Mass transport limitations in heterogeneous catalysis}

Catalysts are ubiquitous in industry. Up to $90 \%$ of the industrial processes employ heterogeneous catalysis. This compelling incentive drives enormous efforts into improving catalytic materials. Extensive experimental data coupled with increasingly powerful ab-initio modelling leads to extraordinary results. However, without a concerted effort regarding reactor design, these exceptional materials cannot deliver their potential. External mixing looses its efficiency close to the surface where mass transport remains diffusion based. To be able to sustain a reaction driven conversion for very high catalytic activities, inspired solutions need to be found.

Heterogeneous catalysis seems condemned to a compromise. High surface

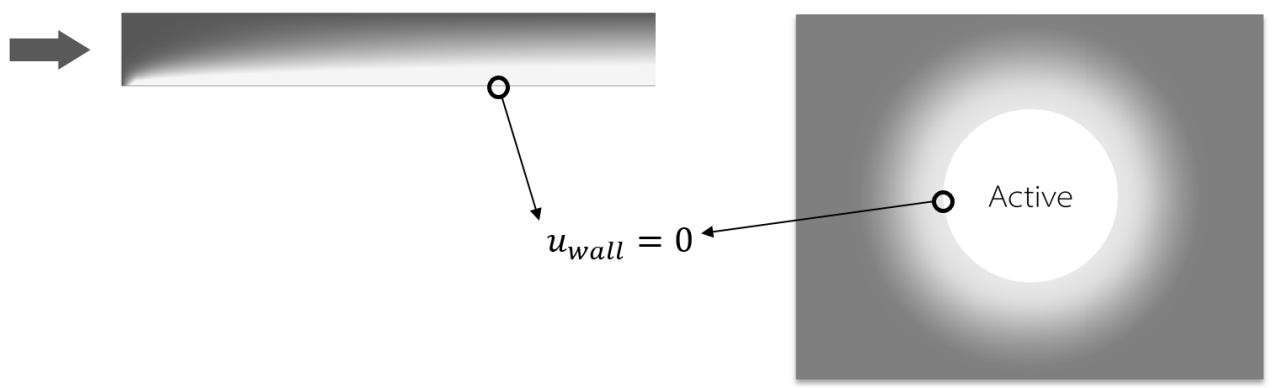

Figure 1.1: Illustration for mass transfer limitation next to active surfaces 

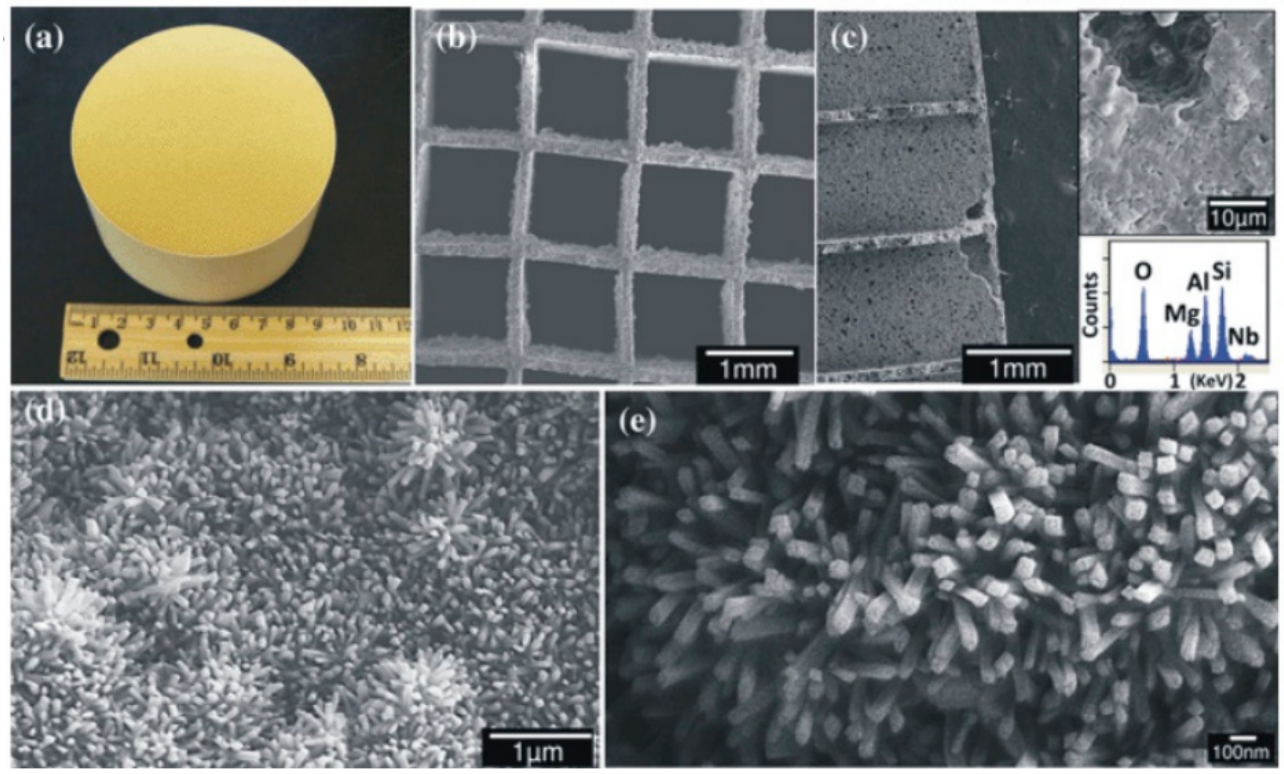

Figure 1.2: Tackling external and internal mass transfer limitations [1]

areas required for the reaction are leading to smaller and smaller structures that provide increasing resistance to mass transport. Chemical engineers have been facing this conundrum for a very long time.

The main strategy is to decrease the diffusion length scale for both internal and external transport. In the case of external transport, this is achieved by distributing the catalyst evenly throughout the reaction medium such is the case of fluidized beds, packed beds or slurry reactors. For fluidized beds and slurry reactors, the catalyst is suspended in the fluid which is mixed or pumped at high flowrates. Additional turbulence can be generated by static mixing geometry and in the case of slurries by bubbling inert gas or making use of potential gaseous reactants or products. For packed beds, the approach is using either smaller catalyst pallets or convoluted structures while finding a trade-off with respect to the corresponding pressure drop increase. The same idea for decreasing the diffusion length scale governs the design of microreactors and monolith structures such as honeycombs and foams. These offer a better control over the pumping energy expenditure. Either straight microchannels or slightly larger channels with additional tortuosity seem to offer a good compromise between external mass transport and pressure drop. 
In the case of internal mass transport, it is generally accepted that diffusion is the only transport mechanism. The only available design option here is to dimension the particle/agglomerate size or layer thickness according to the chemistry at hand. The kinetics will dictate how much of the catalyst is being actually utilised, such that the only intervention is to minimize the material overuse. Well-known parameters such as Thiele modulus and the internal effectiveness factor evaluate this length scale. When the catalyst is embedded in a support matrix, diffusion to the active sites needs to be unhampered, such that open support structures are preferred as the effective diffusion coefficient for the porous matrix will depend on the pore size and tortuosity.

The current thesis challenges this narrative and argues that even when externally driven convection is not available such is the case for transport near the catalyst surface or inside the catalytic matrix, diffusion does not have to be the whole story. Surface flows driven by concentration gradients could be sustained without any energy expenditure. This in-situ generated convective transport opens up additional criteria that could be used for reactor design that would intensify the process beyond the diffusion scenario.

\subsection{Historical background on diffusio-osmosis}

Surface flows generated by concentration gradients and their particle propulsion counterpart have been theoretically speculated by Derjaguin who demonstrated that a solute gradient along a surface will develop an osmotic pressure gradient inside the interfacial layer where the interaction potential spans that will set the fluid in motion (chemi-osmotic contribution). Another contribution to this driving force in case of charged species is the diffusion potential; the electric potential that develops based on the different diffusivities of the ions (electro-osmotic contribution). These individual contributions are illustrated in Figure 1.3. A solid theoretical background has been established from the very beginning with the most significant contributions coming from Derjaguin and coworkers [2-4] and later Prieve, Anderson and coworkers. Prieve and Anderson extended the study of diffusio-phoresis by gradients of neutral species to various molecular interaction potential profiles [5]. In 1984 they showed that the diffusio-phoretic velocity for finite double layers with respect to particle radius depends on the size of the particles, offering clear criteria for the validity of their results [6]. An important contribution is the analysis regarding strongly adsorbing solutes where the outside concentration 


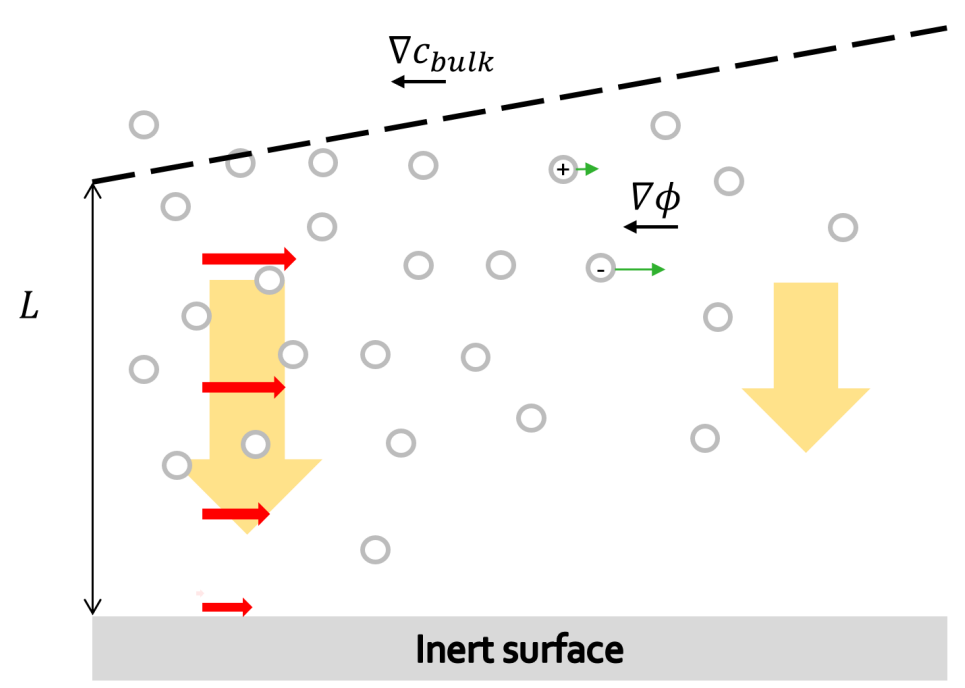

Figure 1.3: A bulk concentration gradient will translate into a diffusio-osmotic flow inside the interaction layer which originates from an osmotic pressure gradient and a diffusion potential in case of charged species with a contrast in diffusivity.

field is affected by the solute transport inside the interfacial layer [7]. They also studied the arbitrary distribution of zeta potential across the surface of the particle, showing that varying surface properties can decelerate the translation movement by the rotation of the particle which aligns the dipole moment of zeta potential along the concentration gradient [8]. Later on, they consider both non-spherical and non-uniformly charged particles with finite double layers [9].

The theory was validated using different experimental designs. Smith and Prieve studied the instantaneous rate of deposition of latex particles on a dissolving stainless steel surface. The dissolution of the metal through the addition of acid and oxidising agent generates a gradient of charged species which develops a macroscopic electric field that acts on the charged latex particles. They showed the linear dependency of the velocity on the local electric field predicted by the analysis of the multicomponent diffusion near the metal surface [10].

However, the mobilities they derived were very small which motivated Lin and Prieve to perform a more controlled study where the deposition of particles on a membrane separating two well stirred containers of different salinity is 
measured for different salts and gradients. They were able to correlate the electric field induced by the difference in diffusivity of the counter ions to the velocity of the particles [11].

Lechnick and Shaeiwitz designed a new experiment to quantify diffusio-phoresis that would remove the uncertainty introduced by the growing particle layer present in the work of Lin and Prieve. They monitored the electrolyte and particle concentration in two well stirred reservoirs of different salinity separated by a thin membrane through which the particles could pass. They validated the theory regarding diffusio-phoresis [12] for monovalent electrolytes, concluded that the theory could be corrected to fit their experimental results for non-symmetric salts and confirmed that the velocity is independent on the particle concentration. They followed up with a second paper where they studied the influence of the electrolyte concentration. Here they showed that for a concentration ratio of the different salinity solutions less than 2 , the average values could be used to accurately predict the velocity [13]. The examples above concern the thin double layer regime. More than three decades later, Shin et al. experimentally probed the effect of the finite Debye layer thickness and confirmed the theory of Prieve on the particle size dependent velocity [14].

Staffeld and Quinn used a more accurate design for studying diffusio-phoresis which they called the stopped-flow diffusion cell where coflowing streams of equal particle concentrations, but different salinity, are suddenly stopped and the transient behaviour of particles is monitored under a microscope for both migration and local concentration [15]. The initial step function concentration profile relaxes due to diffusion leading to an instantaneous velocity that is inversely proportional to the square root of time. The system can be accurately modelled to extract the zeta potential of the particles which they confirm by comparison to a classical measurement. They also made a proof of principle for separating particles based on their zeta potential. A similar study was performed later on in a long microfluidic device by Abécassis et $a l$. who changed the transient analysis to steady state by analyzing the profiles at different locations along the channel $[16,17]$. While the first paper of Staffeld and Quinn addresses electrolyte solutions, the second one investigates the particle-solute interaction in gradients of neutral polymers (Dextran) and charged hard spheres (Percoll). They used the interaction radius as a parameter to describe the step function potential for steric exclusion interaction of hard spheres and an additional electrostatic interaction to account for charge on the surface of the seeding particles as well as Percoll particles [18]. 
Kosmulski and Matuevi followed the particle migration under a gradient of solvent composition by measuring the turbid zone displacement after placing a lower density miscible liquid on top of another which was usually an aqueous particle dispersion. They varied the zeta potential of the particles by changing the $\mathrm{pH}$ and $\mathrm{KCl}$ concentration. Unfortunately, they did not have the possibility to control the gradient and offer only a vague explanation according to which the electrolyte affects the hydration behaviour of the particles [19]. Paustian et al. used a more accurate design to have precise control over the salinity and solvent gradients. They used hydrogel membranes to divide channels with different composition and monitored the transient behaviour of particles after stopping the flow [20]. Nery-Azevedo et al. used the same device for tracking the migration of particles under ionic surfactant gradients [21] while Shi et al. used it under opposing gradients of different electrolytes [22] where the neutralizing reaction leads to focusing of particles.

\subsection{Applications and recent developments}

Once the theory matured and the confidence in the experimental results increased, there was a natural transition from fundamental studies to applications. Numerous proposals were brought forward. Clogging of membranes by particle deposition which is greatly enhanced by diffusio-phoresis could be reduced by insertion of $\mathrm{CaCO}_{3}$ particles. While the chemi-osmotic term will still direct the particles towards the high concentration region, the electro-phoretic contribution, which depends on the relative difference in the diffusivity of the ions, can cancel or even reverse the particle migration by generating a stronger opposing electric field [23-25]. Oil recovery can be enhanced by flooding the reservoir with fresh water which generates salinity gradients and transports the oil droplets out of dead-end pores by diffusio-phoresis [26]. Particles can benefit from the same type of mechanism [27]. Particle separation based on zeta potential and precise particle manipulation [28, 29] have been proven by meticulous design of concentration profiles. Regarding diffusio-osmosis, a very important contribution came from Siria et al. where the diffusio-osmotic flow is measured through a single boron nitride nanotube [30]. Based on their findings, they bring forward the potential of $\mathrm{BN}$ and $\mathrm{TiO}_{2}$ membranes for energy conversion driven by salinity gradients. Later on, Marbach at al. developed a model for both the osmotic and diffusio-osmotic transport through a leaky membrane at high solute concentrations [31]. Diffusio-osmotic flow can also 
be controlled by substrate design as illustrated by Niu et al. which achieved pumping in microfluidics using an ion-exchange resin [32]. Another pumping mechanism is achieved by patterned titania which is activated by UV light [33]. The photocatalytic chemistry consists in the oxidation of water and reduction of oxygen which generates various charged species (hydroxyl radicals, superoxide and protons) whose gradients drive the diffusio-osmoic flow. They also report the use of methanol as fuel to increase its pumping capacity.

Another direction concerns the migration of bimetallic particles that catalyze complementary redox reactions which leads to a distribution of protons and hence an external electric field which electrophoretically drives the particles. This spontaneous electro-chemistry involves the production of charged species that are not being screened by counter-ions, as electrons are transferred through the conductive metals. This phenomenon relies on particular chemicals that decompose spontaneously, namely hydrogen peroxide or hydrazine, used sacrificially as fuel to provide the corresponding transport [34-39]. A short note here is that a combination of metal-nonconductive material leads to the same mechanism. The metal is usually deposited by a directional method which leads to a higher metal thickness at the pole of the particles providing the reaction asymmetry and the polarization within the metal. This phenomenon that is based on a conceptually different mechanism, i.e. through the local net volume charge, relies on a particular chemistry which is rather sacrificial, namely used as fuel to provide the corresponding transport.

An increasing interest is showing up in literature on developing pumping mechanisms without external imposed fields for fine-tuned transport in microfluidic devices or biological systems. There are some efforts beyond electro-chemistry for an envisioned biological compatibility. A nice example concerns the colloidal photodiode where diffusio-osmosis is generated by product concentration gradients [40]. There are two initial solid substrates that decompose into soluble fragments, while one of the products of the first pump is consumed in the decomposition reaction of the second pump. The photolysis of a photo acid generating substrate produces protons that are later on consumed through the hydrolysis of a polymeric imine. Note that the driving force here arises from the high contrast between the diffusivity of protons and the counter ions. Another example of a source only pump is a polymeric film that depolymerizes when exposed to a certain chemical [41]. In this case, the mechanism is based on gradients of neutral solutes.

A few reviews cover examples that focus on microfluidic related applications $[42,43]$, while Velegol et al. puts into perspective the multitude of scenarios 
where diffusio-phoresis spontaneously arises [44]. Diffusio-phoresis plays a role from DNA and virus transport, to bone healing, kidney stone formation and pseudomorphic mineral replacement. They hypothesized on ubiquitous natural phenomena where the stratification of layers of different salinity due to evaporation or incoming streams of melted ice may affect the transport of pollutants.

Technological advancements, especially regarding material science, and the corresponding increased capabilities of experimental design drive new research areas for diffusio-phoresis. Ajdari and Bocquet introduced a new concept regarding enhancement of the slip through solvent repellent surfaces. They do find that the condition for a significant enhancement is a repulsive interaction between the solute molecules and the surface [45]. Michelin et al. conceptualised a microchannel design with an asymmetric sinusoidal profile where opposing walls benefit from the production/consumption synergy to drive flow. The gradients are designed by geometric arrangement rather than activity contrast [46].

The analysis has been extended for charge regulating surfaces, porous spheres, hard spheres with soft shell, soft spheres, liquid droplets and surrounding nonNewtonian fluids. The velocity can double in the case of a shear thinning Carreau fluid with respect to the Newtonian case. If the ion size effect is taken into account, the velocity has been found to be higher than for point charges. Unlike the case of thin double layers where the size, shape and density of particles have no influence on the velocity, when polarization of the double layer is taken into account, the particle-particle interactions have been found to be significant. The interplay between the diffusio-phoresis of particles and diffusio-osmosis of neighbouring boundaries has also been studied. The relaxation of the gradient due to convection and the corresponding decrease of the particle velocity has also been evaluated. A review by Keh summarizes the latest theoretical contributions on both diffusio-osmosis and diffusio-phoresis [47].

\subsection{Diffusio-osmosis induced by catalytic surfaces}

These are valuable applications, but may give the impression that diffusioosmotic flows are very specific. It is either the serendipity of a certain system or a sustained effort to generate the conditions for this flow to arise. Their relevance may also seem restricted by the magnitude of these velocities which 


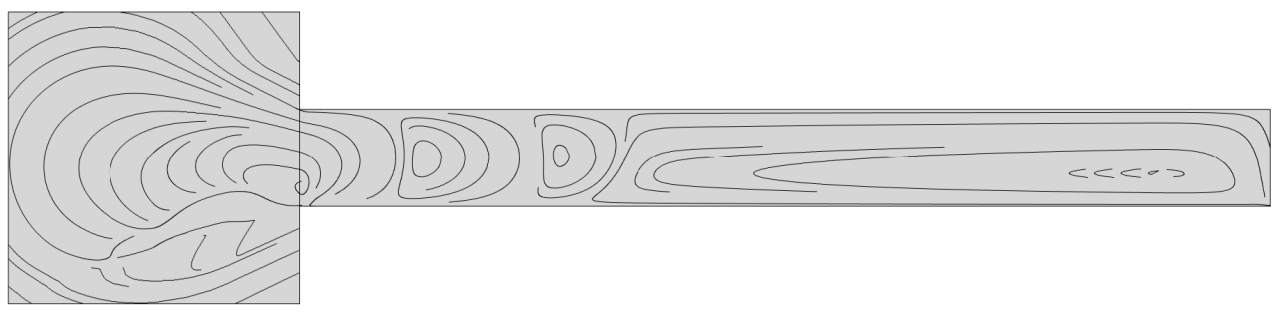

Figure 1.4: Illustration for diffusio-osmotic flow inside a catalytic pore. The recirculation that replenishes the catalytic pore originates from the concentration gradient that develops with respect to the bulk solution.

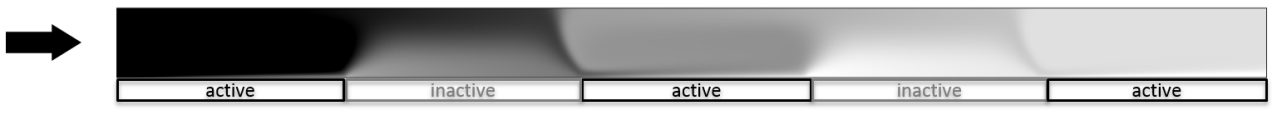

Figure 1.5: Illustration for using surface heterogeneity to enhance external transport. The patterned catalyst generates concentration gradients that drive diffusio-osmotic flow which mixes the boundary layer.

average to few micrometers per seconds peaking for brief transients in the lower range of tens of micrometers per second. Most of the previous examples used either externally imposed gradients or in-situ generated gradients by ion-exchange, dissolution or depolymerization. These all suffer from a finite material capacity (either saturation or depletion of surface). In the absence of a constant driving force, these scenarios are inherently transient, as concentration gradients relax. A steady state which also implies a sustained driving force requires a consumption / replenishment enclosing system.

We propose in this thesis that heterogeneous catalysis can provide a framework for effortlessly generating concentration gradients which are the requirement for driving surface flows. Now, their magnitude can easily surpass $100 \mu \mathrm{m} / \mathrm{s}$ even for moderate kinetics. Moreover, the requirements for maintaining these flows at steady state are in accordance with inherent principles of catalytic reactor design. But what is most important, their impact on mass transport far exceeds their seemingly small magnitudes. The key is location. They arise at the surface of the catalyst without any inconvenience of confined volumes and are inevitably redirected out of plane stirring the otherwise quiescent depleted layer.

We proposes to explore this free transport mechanism in already available 

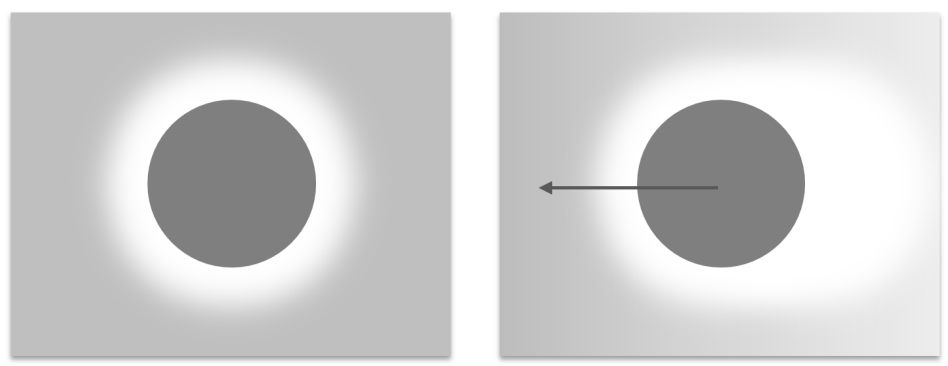

Figure 1.6: Illustration for self-induced diffusio-phoresis by homogeneous catalytic particles. The left side depicts a plain catalytic particle with a surrounding depleted boundary layer. The right side represents the spontaneous phoresis of this particle once a macroscopic gradient develops due to the uneven distribution of other similar particles.

catalytic systems. The spontaneously occurring surface flow can have a great impact on the overall conversion, as interfacial transport is a known limitation for heterogeneous catalysis. The surface reaction can create exceptional steep gradients. Not to forget that in the framework of this application, the continuous supply of reactant is a prerequisite. Furthermore, there is a synergy between the surface flow and the reaction rate with one enhancing the other that is particularly exciting. By providing a direct quantification of the diffusio-osmosis phenomena, the design of catalytic reactors that maximize the benefits of this additional transport mechanism is made possible.

\subsection{Scope of the thesis}

The project investigates the relevance of diffusio-osmosis for the main aspects of mass transport in heterogeneous catalysis: external transport, internal transport and the analogue propulsion of particles by these surfaces flows in the case of suspended catalysts.

To quantify the diffusio-osmotic phenomena we need a framework for investigating the catalytic reactions as previous kinetic information is essential for understanding the dynamics of the system. A microfluidic platform using an inline analysis based on UV-Vis spectrometry proved to be a proper tool due to the well-defined mass transport and fast analysis. 
Chapter 2 starts with an overview on photocatalytic reactor design, focusing on unbiased kinetic investigation. This study encompasses guidelines for heterogeneous catalysis in general, as photocatalysis builds further on the complexity. In this review, the underlying physics of photocatalytic reactions are tackled by providing rational reasoning for simplified analytic descriptions. We start by analyzing the charge carrier generation and transfer, move on to radiative transfer based on the distribution and properties of the catalytic material and account for the mass transfer both inside and outside the porous structure. Finally, we discuss the consequences for the most basic reactor designs for which guidelines and criteria are provided to meet their assumptions.

Chapter 3 presents a complete model for immobilized photocatalytic microreactors and explores their potential to obtain intrinsic kinetics. Accurate modeling for microreactors can be achieved by applying basic physical mechanisms. This leads to a rational reactor design and easy optimization. Models capable of describing reactor performance were build for a first order reaction rate with either light independency or light dependency described by photon absorption carrier generation mechanism. The extracted reaction rate constant reveals the intrinsic kinetics as both external and internal mass transport are accounted for. For the first time $k$ values on the order of magnitude $10^{1} \mathrm{l} / \mathrm{s}$ are reported. The simplification to the light independent model is justified by defining a criterion for neglecting light intensity based on film thickness and absorption coefficient. Performance parameters are also derived for the situation when light absorption has to be considered. The updated internal effectiveness factor reveals both mass transfer and light limitations.

In Chapter 4 a microfluidic platform was developed for high temperature, high pressure conversion to extend the chemistry range that can be explored. The inline UV-Vis spectroscopic measurement facilitates the fast screening of catalytic materials. The well-defined mass transport characteristic for immobilized catalytic layers in microchannels allows for accurate kinetic investigation. One of the essential reactions in the biomass conversion platform, the dehydration of fructose to 5-hydroxymethyl-2-furaldehyde (HMF), was studied using both sputtered $\mathrm{ZrO}_{2}$ and wash coated $\mathrm{TiO}_{2}$ layers. The kinetics were determined for each catalyst. For the $\mathrm{TiO}_{2}$ layer that showed higher conversion, the dependency on temperature was also investigated, revealing an activation energy of $\sim 80 \mathrm{~kJ} / \mathrm{mol}$. Surface functionalization of $\mathrm{TiO}_{2}$ using phosphoric acid treatment under UV light proved to have a limited capacity for increasing the density of active sites. This chapter was initially designed as one of the catalytic reactions to show case diffusio-osmosis. Unfortunately, 
the kinetics for the employed catalytic materials are inherently slow such that these systems do not suffer from mass transport limitations.

In Chapter 5 we numerically study the concept of enhancing external mass transport by patterned catalytic surfaces. The reactivity contrast designed by alternating active and inactive regions spontaneously generate in-plane gradients that drive a steady diffusio-osmotic flow. We use a numerical model based on a first order reaction rate assumption to study the structure of the diffusio-osmotic flow, its development and dependency on the catalytic chemistry, i.e. reaction kinetics and interaction potential between chemical species and catalytic surface (expressed through the mobility parameter). The study reveals that while diffusio-osmosis is initiated by the contrast in reactivity, it develops along the catalyst due to a self-reinforcing mechanism specific to active surfaces. The flow parallel to the catalyst surface introduces a residence time distribution and thus a concentration gradient which is the sustaining driving force that depends on both kinetics, $D a_{I I}$ and mobility, $\mu$. Based on this fundamental understanding we introduce criteria to dimension the catalyst patch according to the chemistry. Scaling laws provide a direct correlation between the catalytic chemistry, the dynamics of the system and the conversion enhancement.

In Chapter 6 we demonstrate that convective transport is characteristic inside catalytic dead-end pores as a result of generated surface flows, solely. These surface flows are induced by concentration gradients that form during catalysis inside the pores. We quantify and explain the onset of diffusioosmosis and discuss its relevance in existing catalytic systems. We visualise and quantify the flow in 3D using the General Defocusing Particle Tracking technique. We analyse the phenomena using a model that includes the fluid dynamics actuated by the concentration gradients that arise due to the catalytic reaction. We are able to extract parameters revealing the interaction strength between the reactant/product chemical species and the catalytic surface. In the end, we probe the dependency of this in-situ generated diffusioosmotic flow on its driving force by varying the reaction rate which changes accordingly the concentration gradients of the reactant and product species.

Chapter 7 follows the diffusio-phoresis of plain catalytic particle induced by macroscopic concentration gradients generated by the particles themselves. The symmetry breaking is designed by the uneven distribution of particles. The migration of photocatalytic particles is studied systematically in a microreactor where an aqueous solution of an organic contaminant is contacted under continuous flow with a particle suspension containing the same solute 
concentration. When UV light is turned on, the photocatalytic particles decompose the contaminant lowering the solute concentration inside the colloidal stream. The difference in concentration that is generated via the photocatalytic reaction leads to the migration of particles toward the higher concentration site. The effect of the reaction rate on the migration of particles is evaluated by changing both the light intensity and initial particle concentration. We explore this migration mechanism by experiments and numerical simulations.

Chapter 8 emphasizes the change in paradigm regarding mass transport in heterogeneous catalytic systems introduced by in-situ reaction induced diffusio-osmosis. A summary of the most important findings of this thesis is provided and the many open questions that remain are examined. A proposal for the experimental approach to these essential investigations is provided. The potential of this phenomenon for fundamental studies is anticipated and industrial perspectives beyond enhanced mass transport are discussed. 



\section{2 Photocatalytic reactor design: guidelines for kinetic investigation ${ }^{\circ}$}

This review addresses the inconsistencies in interpreting measurements of intrinsic catalyst properties using lab-scale devices. Any experiment has to analysed in the framework of a model for which the choice and assumptions regarding the necessary parameters have to be based on critical reasoning. Either through rigorous 3D computational modelling or simplified analytic descriptions, physical intuition about the properties of the system is required. Any divergence between hypothesis and characteristics of the systems affects both the investigation of intrinsic catalytic properties and the later industrial design where parameters are extrapolated outside their obtained operating range. In this work, we make an overview of the underlying physics of photocatalytic reactions, while focusing on pertinent hypothesis and discuss the consequences for the most basic reactor designs for which guidelines and criteria are provided to meet their premise.

\footnotetext{
${ }^{\circ}$ Published as: Aura Visan, van Ommen, J. R.; Kreutzer, M. T.; Lammertink, R. G. H., Photocatalytic Reactor Design: Guidelines for Kinetic Investigation, Ind. Eng. Chem. Res. 2019, 58 (14), 5349-5357.
} 


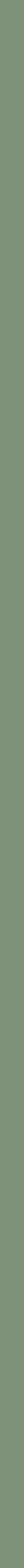




\subsection{Introduction}

Photocatalysis is still seldom used in industry despite the explosive increase in research efforts $[48,49]$. There are two research areas that have too little exchange of information and ideas: catalyst fabrication and reactor design. Unfortunately, these disparate contributions do not amount to industrial development if there is not a shared basic knowledge. This paper is aimed at providing a common ground.

Intrinsic kinetics are of paramount importance when developing new catalytic materials. The comparison across different research groups can be possible only if the performance of the catalyst is decoupled from the reactor design. In this way the best approach, for example, to increase quantum efficiency or material resistance can be spotted early on and a more rational trend can follow. It is far too common that research design is motivated by the history of the group or simply left to chance. While diverging towards new ideas has its indisputable value, serendipity has a statistical disadvantage. Purposeful design exerted by a closely interconnected community is the only way to move forward.

Knowledge of the intrinsic properties of a catalyst are essential to optimizing reactor design. This entails not only tuning the mixing rate or superficial velocity according to the mass transfer requirements. The particle density and spatial distribution required to achieve economically feasible conversions define the optical properties of the system, which are necessary for determining the slurry volume for a given light intensity. Moreover, the dynamics of these slurries can alter the particle distribution and affect their aggregation state which impacts their usage of light. These decisions are not as straightforward as maximizing output, but are also affected by operation and material costs, as well as downstream separation. These decisions have to be taken a priori based on an accurate knowledge of the catalytic material, as changing operating conditions may not be sufficient to render the process economically feasible and encourage industrial implementation.

Intrinsic catalyst properties denote values that are independent of reactor design or are explicitly defined inside the operating range for which their validity holds. For example reaction kinetics should be decoupled from mass transfer and light dependency should be accurately described. If simplifications are sought, their applicability regime should be clearly specified. Optical properties for dispersed systems are based on collective particle characteristics, i.e. 
both particle density and aggregation state. Explicit information of the size distribution and the agglomeration development should be given such that the significance of the measurement is not limited to that particular system, but adds to a broader physical intuition that could in the end lead to predictive models.

The objective of this review is to provide guidelines and criteria for the measurement of intrinsic catalyst properties using lab-scale devices and build on the physical intuition for those who are more comfortable with the mathematical description of the phenomena. We start with the dependency of the reaction rate on the local light intensity. Next, we study how to determine the light intensity across the system based on the distribution and properties of the catalytic material. Finally, the mass transfer is analyzed both inside and outside the porous structure. While the first three sections are presented for clarity separately by decoupling their complexity, in the last section we integrate the most important conclusions in an overview regarding reactor design. We address both suspended small photocatalytic particles and wallcoated films of photocatalysts and present the fundamental designs for ideal systems and the deviations that can still be treated analytically. These basic designs can be employed under certain operation conditions for the most commonly used reactors based on the criteria for these approximations.

\subsection{Electron-hole pair generation}

A photocatalyst is a semiconductor that absorbs photons of equal or higher energy than its band gap which excites electrons from the valence band into the conduction band, leaving positive holes in the valence band of photocatalyst. The generated electrons and holes can migrate to the surface to engage in redox reactions with adsorbed substrates. This is, however, in competition with electron-hole recombination in the bulk or on the surface of the photocatalyst within a very short time, releasing energy in the form of heat or photons [50]. To maximize the reaction efficiency, recombination should be minimized.

Several ways to reduce electron-hole pair recombination (i.e., enhance the charge separation) via modification of the photocatalysts have been proposed in literature. One is to deposit fine noble metals on the photocatalyst surface $[51,52]$. While this is an effective approach, one should be aware that such noble metals might also give a catalytic effect in the absence of light, which can obscure experimental results [53]. Another approach is doping the 
photocatalyst with metal ions. This may both enhance the charge separation and the response in the visible light range, but for some metals (e.g., transition metals) it may also reduce the charge separation $[54,55]$. Coupling two semiconductors with different band gaps is an alternative way to enhance electronhole separation [55]. The effect of particle size on recombination is complex: some authors report a reduced charge separation with reducing particle size from 100 to $10 \mathrm{~nm}$ [56], while others report increased charge separation with reducing particle size down to $10 \mathrm{~nm}$ [57]. Moreover, the effect of doping on the charge separation can strongly depend on the particle size [58]. A complicating factor is that the crystal phase has an influence on charge separation (anatase versus rutile for $\mathrm{TiO}_{2}$ ) but the crystal phase is also influenced by particle size.

The generation and recombination of electron-hole pairs strongly influences the reaction kinetics. There is an ongoing debate about the influence of light intensity and the corresponding regimes. In most photoreactors both regimes coexist: high intensity close to the illumination source and diminishing intensity as light travels through the reactor farther from the source. Thus, a proper kinetic rate expression must take this distribution into account. There are mainly two approaches that we are aware of when deriving the dependency on light intensity, $I$ : the mechanistic approach and the semiconductor physics approach.

In the mechanistic approach a kinetic model is set up based on the law of mass action for both chemical species and electron hole pairs, namely that the rate at which they react is dependent on the diffusion driven collisions which are directly proportional to their concentration. In this case, the recombination rate is defined as $r_{\text {recomb }}=k_{\text {recomb }}\left[h^{+}\right]\left[e^{-}\right]$, where $k_{\text {recomb }}$ is the recombination reaction rate constant, $\left[h^{+}\right]$and $\left[e^{-}\right]$are the positive and negative charge carrier concentrations. The simplification $r_{\text {recomb }}=k_{\text {recomb }}\left[h^{+}\right]^{2}$ can be made for ideal intrinsic semiconductors where the positive and negative charge carriers are in equal concentration. Intentional or unintentional doping will lead to an excess for one of the carriers. This leads to the proportionality of the photocatalytic reaction rate to $r \propto\left[h^{+}\right] \propto I^{0.5}$ when the consumption of holes due to the chemical reaction is negligible compared to the recombination rate, following the charge carrier governing equation: $I \approx r_{\text {recomb }} \approx G$, with $G$ the generation rate [59-61]. The transition of the exponent from 0.5 to 1 is explained by the competition between electron-hole recombination and photocatalytic reactions [62-64]. Given the very small quantum efficiencies (less than $1 \%$ ) for these reactions, we find the assumption unreliable. 
The semiconductor approach starts also from the charge carriers governing equation using the same assumption regarding the negligible consumption of holes and electrons due to reaction, hence $\left[h^{+}\right]\left[e^{-}\right] \approx G / k_{\text {recomb }}$. Here, the generation rate dependency on the local light intensity is directly quantified: $G=\alpha \phi / \hbar \omega$, where $\alpha$ is the absorption coefficient, $\phi$ the photon flux density, $\hbar \omega$ is the photon energy. Light absorption follows the Lambert-Beer law $\phi(x)=\phi_{0} \cdot e^{-\alpha x}$. However, the reaction dependency on the electron/hole concentration is extended beyond the mechanism of an elementary reaction. Nielsen et al. [65] follow the electrochemistry reasoning, namely that the driving force for the reaction rate is the photovoltage, $V_{p h}$, which can be derived based on semiconductor physics:

$$
e V_{p h}=k_{B} T \ln \frac{\left[h^{+}\right]\left[e^{-}\right]}{\left[h^{+}\right]_{0}\left[e^{-}\right]_{0}}
$$

where $e$ is the elementary charge, $k_{B}$ the Boltzmann constant and $T$ the temperature. Moreover, when the photovoltage drives a rate-limiting electron transfer process, the rate depends exponentially on the photovoltage:

$$
r \propto e^{\frac{e V_{p h}}{k_{B} T}}=\frac{\left[h^{+}\right]\left[e^{-}\right]}{\left[h^{+}\right]_{0}\left[e^{-}\right]_{0}}
$$

which gives in the end the following expression:

$$
r \propto \frac{G}{k_{\text {recomb }}\left[h^{+}\right]_{0}\left[e^{-}\right]_{0}}=\frac{\alpha \phi_{0} \cdot e^{-\alpha x}}{k_{\text {recomb }}\left[h^{+}\right]_{0}\left[e^{-}\right]_{0} \hbar \omega}
$$

The denominator is a material characteristic, as well as the light absorption coefficient $\alpha$, which can be measured experimentally using time resolved microwave photoconductivity via the minority carrier lifetime and the equilibrium hole concentration [66].

Nielsen et al. do allow for an exponent smaller then $1, r \propto\left(\frac{\left[h^{+}\right]\left[e^{-}\right]}{\left[h^{+}\right]_{0}\left[e^{-}\right]_{0}}\right)^{\gamma}$, where $\gamma$ is the corresponding transfer coefficient for the electron transfer process. The transfer coefficient is a material characteristic related to its morphology which corrects the model for additional phenomena that were not accounted for. One must be aware that the above derivation is valid for ideal intrinsic semiconductors with a homogeneous crystalline lattice. There are multiple phenomena that involuntarily appear in an experimental system such as trapassisted generation and recombination that arise from crystalline defects (e.g. unintentional n-doping in $\mathrm{TiO}_{2}$ due to oxygen vacancies) or impurities. Not to forget, the surface itself is a severe disruption of the periodic crystal. 
The transfer coefficient is fundamentally different from the exponent in the mechanistic approach and does not depend on the intensity of light. Nielsen et al. also draws attention on the confusion related to the apparent order in light intensity and shows that also for their system if they simply fit the conversion data to a power law equation, $r_{\text {exp. }}=b I_{a p p}^{\gamma}$ they find a variable, the apparent reaction order $\gamma_{a p p}$ that decreases to an asymptotic value for increasing catalyst thickness. This variation in $\gamma_{a p p}$ comes from the interplay between reaction rate and diffusion and therefore, another possible explanation for the experimental findings in literature regarding the variation in the order of light is that for high intensities the reaction rate is fast and diffusion is prevailing, while for low intensities the reaction rate becomes limiting and mass transfer limitations can be neglected. This is also supported in Visan et al. where $\gamma$ remains 1 for a wide range of light intensities due to the accurate modelling of internal and external mass transport [67].

\subsection{Radiative transfer}

The propagation of light in heterogeneous (particulate) systems such as photocatalytic slurries is influenced essentially by two processes: elastic scattering and absorption. Scattering represents a redistribution of light in all directions, but usually with different intensities in different directions (anisotropic) depending on the characteristics of the particles such as refractive index, composition, size distribution, morphology and dynamics (change in orientation). Absorption depends on the local light intensity given by the modified electromagnetic field upon light-particle interactions as explained below.

The most general mathematical representation for the total electromagnetic field in the presence of arbitrary particles is given by Maxwell's equations. Solving even for a single particle is not a trivial endeavour [68]. Such computations are important for optical anisotropic particles or for complex geometries and can also provide insight into the effect of neighbouring particles. This modelling based on fundamental electromagnetic theory provides light scattering properties for realistic systems.

The relevance for photocatalytic dispersed systems is mostly related to the light intensity distribution which can be solved using the scalar radiative transfer equation (RTE). The derivation of RTE from Maxwell's equations in the far field showing the underlying assumptions is covered by Ripoll [69]. The change in light intensity at every location is solved considering the incoming 
light that is the light from the source plus the scattered light coming from other particles, and the outgoing light, namely the scattered light contribution of that particular location and the loss due to absorption. Here, the wavelength-dependent light interaction properties, the spectral volumetric absorption and scattering coefficients, $\alpha_{\lambda}$ and $\sigma_{\lambda}$, as well as the scattering phase function, $p\left(\Omega^{\prime} \rightarrow \Omega\right)$, have to be imported to resolve the spectral radiation intensity, $I_{\lambda, \Omega}(s, t)$ reaching a given point $s(x)$ in space and time $t$, having a given direction of propagation $\Omega$ defined by the polar and azimuthal angles, travelling along distances measured by the spatial parameter $s$.

$$
\begin{gathered}
\frac{d I_{\lambda, \Omega}(s, t)}{d s}+\underbrace{\alpha_{\lambda}(s, t) I_{\lambda, \Omega}(s, t)}_{\text {absorption }}+\underbrace{\sigma_{\lambda}(s, t) I_{\lambda, \Omega}(s, t)}_{\text {out-scattering }} \\
=\underbrace{j_{\lambda}^{e}(s, t)}_{\text {emission }}+\underbrace{\frac{\sigma_{\lambda}(s, t)}{4 \pi} \int_{\Omega^{\prime}=4 \pi} p\left(\Omega^{\prime} \rightarrow \Omega\right) I_{\lambda, \Omega^{\prime}}(s, t) d \Omega^{\prime}}_{\text {in-scattering }}
\end{gathered}
$$

For the rigorous RTE, isotropic scattering and diffuse reflectance phase functions are usually used [70-75]. The accuracy of the solutions is dictated by simplifications made on the scattering spatial distribution function [76]. For symmetry arguments, the six flux [77] and two flux [63,78-80] approximations are utilized the most. The former assumes 3D scattering in the six directions of the Cartesian coordinates, while the latter takes into account only forward and back-scattering.

Scattering is always detrimental to the overall energy absorption. Since the scattered light is not lost for the system, but merely contributes to other directions, the change in direction inside the reactor could be intuitively understood as an overall decrease in the optical path. The change in direction inside the reactor will limit the penetration distance accordingly. The change in optical path for different scattering models is illustrated in Figure 2.1 by the absorbed light fraction, $\Psi$, solved for different scattering albedos, $\omega$, which represents the ratio between the scattering coefficient and the sum of scattering and absorption coefficients. Light absorption is underestimated to the greatest extent in the two flux model due to the highest optical path decrease coming from considering only back-scattering.

We argue that a more rational approach should be sought. The coherence between modelling and the properties of the particles is usually missing in the photocatalytic literature. Building a physical intuition is a prerequisite towards understanding the dominant characteristics of each system and provides 


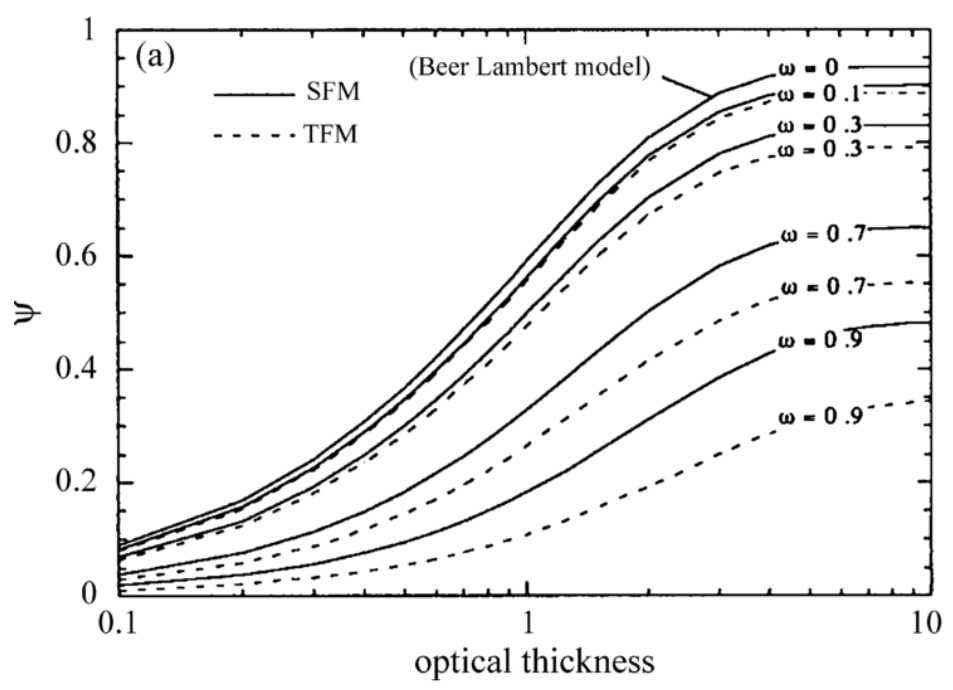

Figure 2.1: Absorbed light fraction vs. optical thickness for different scattering albedos. The dashed lines indicate results predicted by the two flux model (TFM), while the continuous lines depict the six-flux model (SFM). [77]

arguments for simplifying the general RTE. Moreover, intrinsic optical properties, namely the real and imaginary parts of the complex refractive index, $n$ and $k$, cannot be measured directly, but must be derived from measurable quantities. The measured quantities have to be interpreted in the framework of the RTE model to generate the necessary coefficients [81-85]. In order to fit the measured quantities, external inputs such as the scattering phase function are required.

That is why a good starting point for evaluating the optical properties of individual particles is the scattering angular dependency with the particle size. Useful guidelines are provided by known solutions for Maxwell's equations solved for different limiting cases such as scattering by homogeneous spheres (Mie scattering). The scattering phase function is illustrated with polar plots in Figure 2.2 where $x$ is the normalized diameter

$$
x=\frac{\pi \cdot d \cdot m_{\text {medium }}}{\nu}
$$

with $d$ is the diameter of the particle, $m_{\text {medium }}$ is the refractive index of the non-absorbing surrounding medium and $\nu$ is the wavelength. As particles become larger $(x>3)$, isotropic scattering changes to a preferential forward 


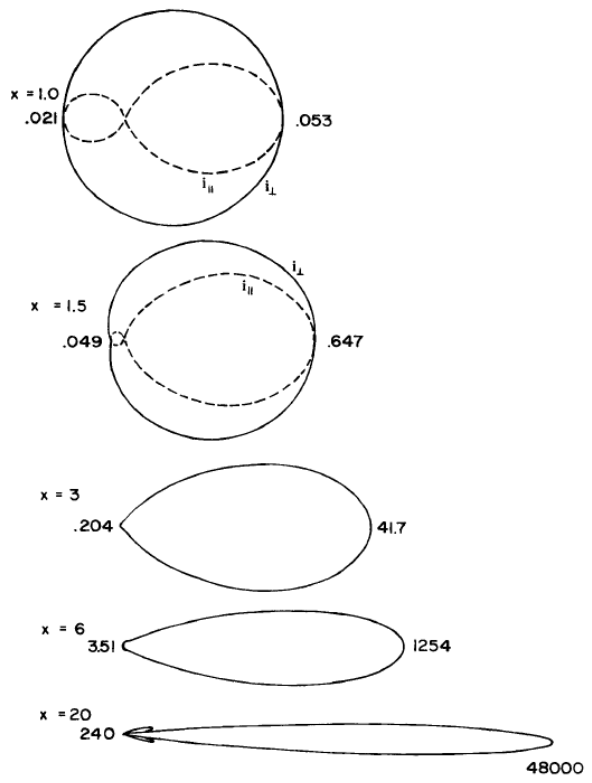

Figure 2.2: Polar plots of the scattering phase function for different particle sizes with $m_{\text {particle }}=1.33$ and $m_{\text {medium }}=1$. [86]

direction.

The scattering magnitude can also be quantified using the scattering cross section which represents the area that would capture the energy of the incident beam equal to the total energy that is scattered in all directions. The corresponding normalized parameter is the scattering efficiency which equals to the scattering cross section divided by the particle cross section area projected onto a plane perpendicular to the incident beam. The scattering efficiency as a function of particle diameter is illustrated in Figure 2.3 for $\mathrm{TiO}_{2}$ particles. Even if the scattering efficiency is presented for $560 \mathrm{~nm}$ wavelength due to the visible range interest for the coating industry, a general trend can be noticed. Scattering is negligible for particle sizes much smaller than the wavelength, $\mathrm{x}<0.6(0.16 \mu \mathrm{m}$ in Figure 3), while maximum scattering is achieved when particle sizes approach the wavelength. The scattering efficiency levels off at a value 2 for larger particles, $\mathrm{x}>3(0.8 \mu \mathrm{m}$ in Figure 2.3), which is characteristic for the geometric scattering regime. A general observation is that large particles scatter twice more light than it is geometrically incident upon them. The scattering coefficients selected from literature should correspond to the 


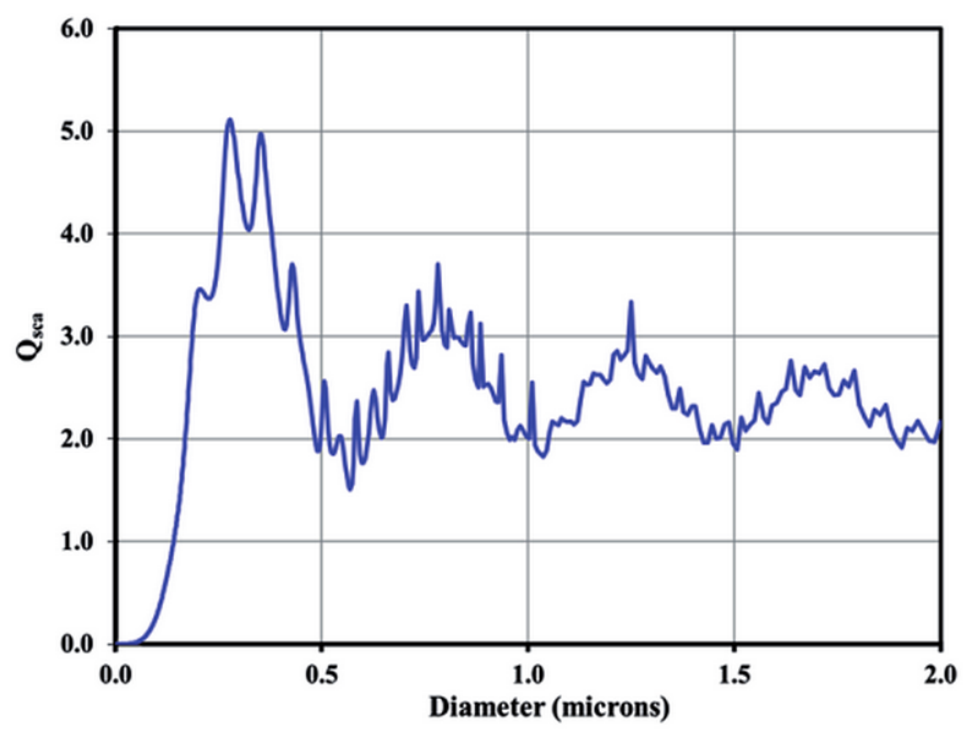

Figure 2.3: Scattering efficiency vs. $\mathrm{TiO}_{2}$ particle size $\left(m_{\text {particle }}=2.73\right)$ embedded in resin $\left(m_{\text {medium }}=1.5\right)$ at $560 \mathrm{~nm}$ [87]. The ripple structure comes from constructive and destructive interference between incident and forward scattered light.

particle size regime of interest and special care should be given to the radiative transfer model used to extract these optical properties from measurements. A poor choice for external inputs such as the scattering phase function can lead to erroneous coefficients which propagate into further modelling.

Dispersed systems have an inevitable degree of agglomeration. Agglomerate sizes in slurries are above $100 \mathrm{~nm}$, in other words, above the negligible Rayleigh regime. Therefore, the lower particle size range for practical applications corresponds to the highest degree of scattering. Nevertheless, the experimentally observed strong light attenuation for fine particle slurries is still related to the efficient absorption of light by the highly dispersed system. For smaller particles, absorption is always the dominant process due to the higher probability of light-particle interaction. However, larger agglomerates with forward scattering are more prominent in realistic conditions. As aggregation develops, the decrease in absorption is independently accompanied by a decrease in scattering. It is a general misunderstanding that the decrease in absorption is due to enhanced scattering. As the particle size increases, the main reason for lower absorption efficiencies is the shadowing effect. The exponential decay 


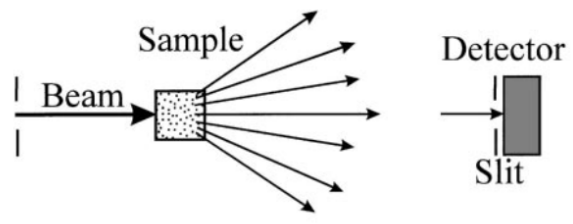

(a)

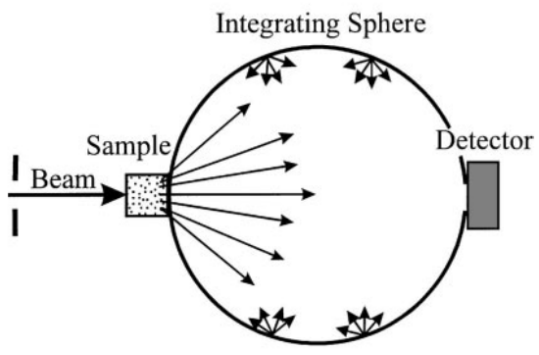

(b)

Figure 2.4: Configurations for measuring absorption and scattering coefficients. (a) Collimated transmittance; (b) Diffuse transmittance. [71]

of light inside the particle agglomerate renders smaller internal effectiveness factors [67] (see reactor design section). Most of the light is then absorbed by only the outer agglomerate material.

While the accurate interplay between scattering and absorption is given by rigorously solving the RTE, simplifications can be used under certain conditions. Decoupling scattering and absorption processes can be verified by observing how attenuation scales with particle concentration. If a linear proportionality exists, then the scattering of neighboring particles does not add up to the light reaching every particle. The important implication concerns cumulative scattering. Scattering of individual particles can be summed up due to no interference from neighboring particles, namely photons are scattered only once. This is a valid assumption for relevant particle concentrations in photocatalysis, which are on the order of a few grams per liter. Crowding effects are noticed only when the distance between particles decreases below 3 times the particle radius [88]. An important consequence is that the exponential profile for attenuation of light is preserved $\left(\phi(x)=\phi_{0} \cdot e^{-\beta x}\right)$. Essentially, the scattering coefficient, $\sigma$ and absorption coefficient, $\alpha$ can be summed up 
into an attenuation coefficient, $\beta$. If this independent absorption hypothesis holds, there are multiple resources that discuss the implications of deviating from ideal systems such as size distribution, various morphologies or optical anisotropy [86].

Collimated and diffuse transmittance experiments illustrated in Figure 2.4 are the standard methods for measuring absorption and scattering coefficients. The challenge is that these coefficients cannot be measured independently in slurry systems. Even in the diffusive transmittance mode, the collected light excludes the back scattered light, so it does not amount to a true absorbance measurement. We propose that for particles which are touching, as is the case for immobilized porous layers, scattering can be neglected. If only the near field interaction of a second particle is considered, a significant $20 \%$ decrease in scattering is observed [68]. Even if some degree of scattering remains inside the porous film due to consecutive transitions between the two different refractive index mediums, this redistribution of light does not lead to external losses. There is a high probability that it will be captured by the densely packed particle matrix. The high solid volume fraction also ensures a strong absorption which makes the relative contribution of scattering to the total attenuation insignificant.

Given the negligible scattering for immobilize layers, a simple transmittance measurement reveals directly the material absorption coefficient based on the solid volume fraction. This could then be easily translated to the absorption coefficient of the slurry systems for different particle concentrations. An available example in the literature defends our reasoning. The absorption coefficients for immobilized $\mathrm{TiO}_{2}$ layers has been reported for both dense and porous films. Looking at the $300 \mathrm{~nm}$ wavelength, the absorption coefficient in the case of dense layers is between 0.033 and $0.0581 / \mathrm{nm}$ [89], while for the porous layer with a 0.45 solid fraction the value is $0.0231 / \mathrm{nm}$ [67]. The range for the former study stems for different synthesis conditions which lead to a variation in the crystalline phase composition. If we follow the previous suggestion and normalize the absorption coefficient by the solid volume fraction, the absorption coefficients in the 2 references become very close, namely 0.06 - 0.03 versus $0.051 / \mathrm{nm}$, revealing its intrinsic value. 


\subsection{Mass transport}

In chemical reactors, a proper description of the mass transport is crucial to determine limitations and obtain the conversions of reactants and formation of products. In photocatalytic processes, light intensity distribution as well as mass transport can become limiting in terms of the overall conversion. In this section, we will briefly treat the relevant steps in mass transport, starting inside a porous structure, moving to the boundary layer at the surface of the structure and finally to convective transport in the bulk.

\subsubsection{Internal mass transport}

We will first consider the mass transport inside a porous structure. In the framework of this paper, this can be an agglomerate of nanoparticles in a slurry system or a porous immobilized catalyst layer. This internal mass transport is governed by diffusion and reaction only at given $x$ :

$$
D_{e f f} \frac{\partial^{2} c}{\partial y^{2}}-r=0
$$

with the following boundary conditions for a slab geometry. At $y=-\delta$, $D_{e f f} \frac{\partial c}{\partial y}=0$ and at $y=0, c=c_{s}$, where $r$ is the reaction rate, $D_{e f f}=D \cdot \frac{\epsilon}{\tau}$ is the effective diffusion coefficient, $D$ is the molecular diffusion coefficient, $\epsilon$ is the porosity, $\tau$ is the tortuosity and $\delta$ is the catalyst thickness.

The equation can be solved analytically for a first-order reaction rate $r=$ $k \cdot c$, where $k$ is the rate constant. This is an effective bulk reaction rate often assumed for simplicity, representing the surface reactions taking place inside the porous material. The concentration profile and the net reaction rate (inward flux) are:

$$
\begin{gathered}
c(y)=c_{s} \frac{\cosh (\phi(1+y / \delta)}{\cosh (\phi)} \\
N_{y=0}=D_{\text {eff }} \frac{\partial c}{\partial y}=c_{s} \frac{D_{\text {eff }}}{\delta} \phi \tanh \phi
\end{gathered}
$$

where $c_{s}$ is the surface concentration at the particle-liquid interface. It is important to realise that this surface concentration can be different from the 
bulk in case external mass transfer limitations are present, as will be discussed later.

The dominating mechanism can be evaluated using the Thiele modulus, $\phi$, and the internal effectiveness factor, $\eta$, defined in Table 2.1 for a first order reaction rate. The former computes the ratio between the reaction and the diffusion time scales, while the later gives the ratio between the net reaction rate and the surface reaction rate, namely the rate in the absence of concentration gradients.

\begin{tabular}{l|c|c} 
Geometry & Thiele modulus & Internal effectiveness factor \\
\hline Slab & $\phi=\sqrt{\frac{k}{D_{e f f}}} \cdot \delta \mid$ & $\eta=\frac{\tanh \phi}{\phi}$ \\
Spherical & $\phi=\sqrt{\frac{k}{D_{e f f}}} \cdot R_{p} \mid$ & $\eta=\frac{3}{\phi^{2}}(\phi \operatorname{coth} \phi-1)$
\end{tabular}

Table 2.1: Performance parameters

Weisz and Prater established that $\phi \leq 10^{-1}$ to neglect or avoid concentration gradients ( $\leq 5 \%$ deviation from a flat concentration profile). The formal criterion [90] for a first order reaction is:

$$
\frac{r_{n e t} R_{p}^{2}}{c_{s} D_{\text {eff }}}<0.6
$$

where $r_{n e t}$ is the net or observed reaction rate. An even more practical meaning is conveyed by the internal effectiveness factor, which directly expresses the fraction of the catalyst that is being utilised. Thiele modulus and internal effectiveness factor are also derived for reaction rates taking into account their dependency on the local light intensity. For clarity, these will be presented in the reactor design section.

\subsubsection{External mass transport}

The reaction-diffusion equation can be solved for boundary conditions that specify the concentration at the catalyst surface which can be determined only if external transport is known. Equality between the surface and bulk concentrations implies perfect mixing, but even in a well-stirred volume this 
is typically not reached for fast catalytic conversions: depletion of reactants at the catalyst surface is still taking place. The easiest method to incorporate this additional resistance to mass transport is to use a stagnant boundary layer model which connects the bulk concentration to the surface concentration via the mass transfer coefficient, $k_{m}$. The flux continuity boundary condition is:

$$
N_{y=0}=k_{m}\left(c_{b}-c_{s}\right)=r_{n e t} \cdot \delta
$$

This flux continuity matches the mass transport through the boundary layer to the total conversion inside the porous catalyst. Using the definition of the internal effectiveness factor, $r_{n e t}=r_{s} \cdot \eta=k \cdot c_{s} \cdot \eta$, and solving the equation for the unknown surface concentration, the net reaction rate becomes:

$$
r_{n e t}=\frac{\eta k c_{b}}{1+(\phi \tanh \phi) / B i_{m}}
$$

where the mass Biot number evaluates the ratio between the internal and external mass transport coefficients, $B i_{m}=\frac{k_{m}}{D_{e f f}} \delta$. A straightforward criterion for assessing the effect of external transport on the reaction rate was introduced by Carberry [91]. For

$$
\frac{\eta k}{k_{m} A}<0.1
$$

the reaction rate constant derived from the observed reaction rate reaches its intrinsic value, where $A$ is the external surface to volume ratio $(1 / \mathrm{m})$. The mass transfer coefficient depends on velocity and can be determined experimentally with the benzoic acid dissolution method [92] or can be computed via empirical correlations [93]:Sh=f(Sc,Re) with $S h=\frac{k_{m} d_{p}}{D}, S c=\frac{\nu}{D}$, $R e=\frac{\bar{u} d_{p}}{\nu}$, where $\nu$ is the kinematic viscosity, $d_{p}$ is the diameter of the particle agglomerates and $\bar{u}$ is the superficial velocity of the fluid. Welty and Wicks [94] give a comprehensive list of convective mass transfer correlations for various types of reactors and operating conditions.

\subsubsection{Convective transport}

Until now, we have worked under the assumption of a constant bulk concentration. However, the replenishment of the bulk solution is not instantaneous and needs to be accounted for a temporal and/or spatial development of the bulk concentration. The concentration in the bulk of the reactor is determined 
by the velocity profile, which influences both the residence time distribution as well as the mass transfer capacity. Following the general approach of the paper, we will seek to simplify the daunting transient 3D analysis to a 1D model. The most basic reactor design is the plug flow model, assuming zero axial dispersion and infinite radial dispersion. The velocity is assumed constant along the axial direction, resulting in:

$$
u \frac{\partial c_{b}}{\partial x}=r
$$

where $c_{b}$ depends only on $x$ since the radial concentration gradients are localized within the boundary layer. Lumping both internal and external mass transport limitations into an apparent reaction constant $k_{a p p}$ :

$$
k_{\text {app }}=\frac{\eta}{1+(\phi \tanh \phi) / B i_{m}} \cdot k
$$

$c_{b}$ has the well-known expression: $c_{b}=c_{0} e^{-k_{a p p} \cdot \frac{x}{u}}$.

This model is equivalent to an ideally stirred batch reactor where the position along the plug flow reactor correspond to a residence time, $\frac{x}{u}=t$.

The plug flow reactor (PFR) is relevant in practice for a continuous operation of a slurry reactor or an effectively mixed immobilized reactor. Concentration gradients can be accounted for by using deviations from plug flow. The axial dispersion approximation can be used to evaluate molecular and turbulent mixing:

$$
D_{a} \cdot \frac{\partial^{2} c_{b}}{\partial x^{2}}-u \cdot \frac{\partial c_{b}}{\partial x}=r
$$

where the axial dispersion coefficient $D_{a}$ can be experimentally determined from residence time distribution measurements [90] or derived using empirical correlations. Fortunately, Eq. (2.15) has analytical solutions for zeroth and first order reaction rates. Using the first order reaction rate model in Eq. (2.11) gives the following $c_{b}$ profile:

$$
c_{b}=c_{0} \frac{4 \cdot \sqrt{1+\frac{4 D a_{I}}{P e}} \cdot e^{\frac{P e}{2}\left(1-\sqrt{1+\frac{4 D a_{I}}{P e}}\right)}}{\left(1+\sqrt{1+\frac{4 D a_{I}}{P e}}\right)^{2}-\left(1-\sqrt{1+\frac{4 D a_{I}}{P e}}\right)^{2} \cdot e^{-P e \sqrt{1+\frac{4 D a_{I}}{P e}}}}
$$

where Péclet number is $P e=\frac{\bar{u} \cdot x}{D_{a}}$ and the first Damköhler number is $D a_{I}=$ $\frac{k_{a p p} \cdot x}{\bar{u}}$ with $\bar{u}$ being the average velocity. The axial dispersion model can be used only for $P e>20$ [95]. 


\subsection{Reactor design and operation}

In the last section, we discuss the underlying assumptions of basic reactor designs and provide criteria that ensure these conditions are accurately met in practice. Here, we integrate the analysis of the physical phenomena presented separately in the first three sections.

\subsubsection{Dispersed systems}

Slurry reactors are widely used in photocatalysis. Such reactors is obtained by dispersing photocatalyst nanoparticles in aqueous environments. These particles are often already aggregated because of their production process (e.g., combustion synthesis for $\mathrm{TiO}_{2} \mathrm{P} 25$ ), and agglomerate even further to larger clusters. These agglomerates typically have a size in the order of $1 \mu \mathrm{m}[71,96]$ and a very open structure. To verify if internal mass transfer can be ignored, a sample from the catalyst aggregates could be redispersed for instance using ball milling. The conversion under identical conditions should be similar before and after crushing. To work under the assumption of an ideally stirred system, the mixing rate should be increased until no further change in conversion is noticed. In this case, external mass transfer can also be ignored, and a homogeneous concentration of reactants and products throughout the system can be assumed. Another type of dispersed system is the stagnant slurry reactor. Here, in the absence of convection, the underlying physics encompass diffusion and reaction only, such that the mathematical description resembles the immobilized catalyst case.

When designing a photocatalytic slurry reactor for optimum determination of reaction rates, one wants to have a nearly constant light intensity inside the catalyst agglomerate and throughout the reactor. The criterion for neglecting light dependency is defined as the decay length in Visan et al. [67] for more than $1 / e$ transmission corresponding to $\delta<\frac{1}{\alpha}$ and in Motegh et al. [63] for less than $5 \%$ deviation of photoreaction rate per particle from the maximum photoreaction rate in the absence of shielding, corresponding to $\delta<\frac{0.1}{\alpha}$. To illustrate the concept, a $\mathrm{TiO}_{2}$ layer with a porosity of 0.45 has a characteristic decay length for the light intensity of $\sim 1 \mu \mathrm{m}[67]$. In a single agglomerate of $\sim 1 \mu \mathrm{m}$ the porosity is much higher, thus it is reasonable to assume a constant light intensity within the agglomerate: i.e., each individual particle is equally 
exposed to the light.

A more general criterion that takes into account multiple scattering is that for common values of the scattering albedo (around 0.7), one has to work at an optical thickness below 0.2 to be able to volume-average the reaction rate [63]. When designing a photocatalytic slurry reactor for maximized use of photons, the optical thickness should be at least 3.5 for low photon fluxes or 6.5 for high photon fluxes. In that case less than $5 \%$ of the photons leave the reactor unused [63]. These threshold values are only a weak function of the scattering albedo.

The guidelines above are for two-phase systems: fine particles dispersed in a liquid. However, Motegh et al. [80] showed that the same guidelines can be used for slurry reactors with gas bubbles, i.e. three-phase systems. For a gas fraction below $20 \%$ and bubble diameters around $3 \mathrm{~mm}$, typical values in such reactors, the effect of the additional scattering by the bubbles on the photoreactor performance is insignificant, and the same limiting values for optimal thickness apply.

\subsubsection{Immobilized systems}

There is a general preference for dispersed catalytic systems due to their enhanced mass transport capacity, as the small inter-particle distance ensures a small diffusion length scale. However, the additional separation step and the corresponding complexity for continuous operation motivate the use of immobilized systems. Moreover, slurry systems have inherently lower quantum efficiencies, as various degrees of scattering are unavoidable.

The general approach for preserving high mass transfer rates for immobilized systems is either to operate at high flowrates which generates strong turbulence, being aided sometimes by static mixers, or reactor design for sudden changes in flow direction or to maintain a small diffusion length scale by decreasing the transverse dimension of the flow channel. In cases where mass transport in the liquid is affecting the conversion, quantification of this transport is required in order to obtain intrinsic kinetics for the catalytic conversion. The former case, namely the well-mixed reactor with immobilized layer can be modelled as a plug flow reactor where the radial mass transfer resistance is represented by a fictitious stagnant layer. For the higher range of velocities, the departure from ideality such as concentration gradients in the axial direction can be handled by extending the PFR to the axial dispersion model. 
In the latter, we refer to microreactors which can be modelled accurately due to the well-defined underlying physics and geometry. The characteristic dimensions ensure a parabolic velocity profile and a homogeneous light intensity across the surface of the reactor. For these reasons microreactors with an immobilized porous layer of photocatalyst on one or both of the channel walls, are attractive devices to obtain the intrinsic kinetics [67]. As described previously, for immobilized layers scattering can be neglected (see radiative transfer section).

Light-dependent kinetics can be derived for intrinsic semiconductors based on their fundamental physics [65]. For the diffusion-reaction regime, lightdependent kinetics can also be tackled analytically. Furthermore, a corresponding internal effectiveness factor has been derived [67] which is evaluating the catalyst coverage by taking into account both mass and radiative transfer limitations:

$$
\eta=\frac{-1}{\delta} \cdot \frac{I 1\left(\frac{2 \phi \sqrt{e^{-\gamma \alpha \delta}}}{\gamma \alpha \delta}\right) \cdot K 1\left(\frac{2 \phi}{\gamma \alpha \delta}\right)-K 1\left(\frac{2 \phi \sqrt{e^{-\gamma \alpha \delta}}}{\gamma \alpha}\right) \cdot I 1\left(\frac{2 \phi}{\gamma \alpha \delta}\right)}{I 0\left(\frac{2 \phi}{\gamma \alpha \delta}\right) \cdot K 1\left(\frac{2 \phi \sqrt{e^{-\gamma \alpha \delta}}}{\gamma \alpha \delta}\right)+K 0\left(\frac{2 \phi}{\gamma \alpha \delta}\right) \cdot I 1\left(\frac{2 \phi \sqrt{e^{-\gamma \alpha \delta}}}{\gamma \alpha \delta}\right)}
$$

where a modified Thiele modulus was computed as the ratio between surface reaction rate and the diffusion rate:

$$
\phi^{2}=\frac{k_{i} \cdot \delta^{2}}{D_{e f f}} \cdot\left(\frac{\alpha \Phi_{0}}{B p_{0} n_{0} \hbar \omega}\right)^{\gamma}
$$

where $B p_{0} n_{0} \cong 3.3 \cdot 10^{-23} \mathrm{~m}^{-3} \mathrm{~s}^{-1}$ is the equilibrium electron-hole recombination rate [65]. $\mathrm{I}_{n}(\mathrm{x})$ and $\mathrm{K}_{n}(\mathrm{x})$ are modified Bessel functions of the first and second kind, respectively.

\subsection{Conclusions}

In this review, we tackle the underlying physics of photocatalytic reactions by providing rational reasoning for simplified analytic descriptions. We begin by analyzing the charge carrier generation and transfer, move on to radiative transfer based on the distribution and properties of the catalytic material and account for the mass transfer both inside and outside the porous structure. 
Finally, we discuss the consequences for the most basic reactor designs for which guidelines and criteria are provided to meet their assumptions.

For ideal intrinsic semiconductors, the photocatalytic reaction rate scales linearly with light intensity if mass transfer is accurately accounted for. Simplifications to the radiative transfer equation should start based on a physical understanding of the scattering angular dependency on the size of the particles with respect to the wavelength. The particle size regime can also give an idea about the scattering magnitude. It is only in the framework of pertinent assumptions that the experimental measurements can be correctly interpreted to provide true optical properties. The validity of simpler models, such as exponential attenuation of light, could be confirmed for a certain particle density regime by testing the proportionality between particle concentration and light attenuation. Immobilized films benefit from negligible scattering with simple transmission measurements revealing true absorption coefficients that can be used in dispersed systems to decouple absorption from scattering. The reaction-driven regime should be established using criteria such as Thiele modulus, internal effectiveness factor and Carberry criterion or mass transport limitations should be properly described using for example the stagnant boundary layer model or the axial dispersion model. Empirical tests are also presented to discard both external and internal mass transfer limitations.

This review should be of assistance in designing experiments where the underlying assumptions for various photocatalytic models are accurately met and reveal the operating condition range for which these values are still accurate. Those who deal with rigorous 3D computational modelling can foresee the possible complexities for certain creative designs that may venture outside the experimental data set and care can be exercised when trying to extrapolate. Having a good grasp of the intricacies of the system, they can identify the missing parameters and establish a dialogue with experimentalists. This will aid in creating a situation in which the experimental endeavors are directed purposefully by investigating better defined questions from researchers in complementary fields. 



\section{3 Modeling intrinsic kinetics in immobilized photocatalytic microreactors ${ }^{\circ}$}

The article presents a simple model for immobilized photocatalytic microreactors following a first order reaction rate with either light independency or light dependency described by photon absorption carrier generation semiconductor physics. Experimental data obtained for various residence times, catalyst thicknesses and photon flux densities proved that the model is capable of describing the reactor performance. The extracted reaction rate constant reveals the intrinsic kinetics as both external and internal mass transport are accounted for. The effect of light is also considered by defining a criterion for neglecting light intensity based on film thickness and absorption coefficient. For the first time $k$ values on the order of magnitude of $10^{1} 1 / \mathrm{s}$ are reported. In the end, performance parameters are also derived for the light dependent model for which the internal effectiveness factor reveals both mass transfer and light limitations.

${ }^{\circ}$ Published as: Aura Visan, Rafieian, D.; Ogieglo, W.; Lammertink, R. G. H., Modeling Intrinsic Kinetics in Immobilized Photocatalytic Microreactors, Appl. Catal. B Environ. 2014, 150-151, 93-100. 


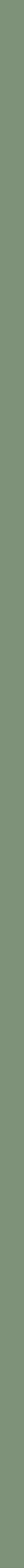




\subsection{Introduction}

Heterogeneous photocatalysis is a promising technology for environmental remediation. However, commercial products are focused mostly on self-cleaning surfaces and air filtering. Important applications such as removing refractory compounds from wastewater are scarce. In 2007 van Gerven et al. [48] mentioned only 6 examples: Zentox Corporation, Matrix Photocatalytic Inc., Clearwater Industries, Photox Bradford Ltd., Lynntech Inc. and Purifics Environmental Technologies Inc. The invoked reasons are small conversion capacities and inefficient light use, addressing especially the inconsistencies in the definitions of various performance parameters. A progress in optimization requires that individual research efforts can be combined which is possible only if an honest comparison between them can be achieved.

One parameter used frequently when evaluating the conversion capacity of a reactor is the apparent reaction rate constant. Its derivation implies identifying the reaction rate with the conversion $-d c / d t=r$. The kinetic model is usually Langmuir-Hinshelwood $r=\frac{k K c}{1+K c}$ or a first order reaction $r=k c$ from which the reaction rate constant is obtained [97-105]. The agreement with respect to these basic relations is widespread, as several reviews mention it $[106,107]$.

Heterogeneous reactions involve the diffusion of species to the active catalyst sites. Mass transport becomes important and if omitted can alter the true values of the reaction kinetics. Intrinsic kinetic parameters are of paramount importance. They are required for catalyst screening and necessary when simple engineering tools such as the apparent reaction constant are used for comparing different reactor configurations. The real value for the reaction rate constant allows the evaluation of the mass transfer contribution on the conversion which is a main factor dictating the performance of a reactor. Evidently, comparison complicates further when the gradients in photon absorption become important and kinetics cannot be volume averaged anymore. When both immobilized and dispersed systems are considered, the difference in light dispersion can be bridged only by light dependent intrinsic kinetics.

The standard approach for kinetic investigation is to place the system in the reaction rate limiting regime, so the apparent reaction rate can reach the intrinsic value. The classical method uses a differential reactor which consists of a reaction chamber and a mixing tank, where the reaction volume is much smaller than the total volume. The small conversion per pass allows the sim- 
plification of the mass balance to a batch reactor equation. The intrinsic kinetics are determined for flowrate - independent conversions [108-119]. Accepting the reliability of this method to eliminate external mass transport, the question about internal mass transfer remains, which will be present even for thin catalyst layers or for an inevitable degree of aggregation in slurry systems. Ballari et al. [120-122] went on to defining guidelines for canceling mass transfer limitations in slurry reactors based on flow rate, catalyst loading and irradiation rates.

Even when concentration gradients are eliminated, the inhomogeneity regarding light distribution has to be carefully considered, especially for slurry systems. Motegh et al. [63] gave guidelines for operating in optically differential mode. Starting from the premise of perfect mixing, a criterion was defined for keeping the gradients in photon absorption rate small enough as to allow the volume-averaging of the reaction rate.

A more thorough method is to model light distribution, fluid dynamics and mass transfer and fit the kinetics to the experimental data. Due to the complexity of large-scale photocatalytic reactors, a lot of assumptions come into play.

The first challenge in a large-scale system is the non-uniform incident flux. To obtain the radiation field distribution, light emission models have to be correlated to the radiation transfer equation (RTE) in order to obtain the local volumetric rate of energy absorption (LVREA) which can be afterwards coupled to the reaction rate. In case of dispersed systems, the radiation transfer equation becomes more complex due to in and out-scattering effects that also depend on the aggregation extent of the particles [79,123-126].

The next step is to consider the complex hydrodynamics. The most rigorous approach is to perform a CFD simulation which solves the continuity and Navier-Stokes equations and also requires turbulence models. Again, dispersed systems demand the most elaborate models. An Eulerian multi-fluid approach is necessary to connect the fluid velocity field to the solid particle distribution. However, real flow computations are quite challenging. This is why, when possible, approximations are used. In case of small deviations from laminar flow, the axial dispersed model can be considered. For this, the Péclet number (Pe) can be experimentally determined from RTD measurements $[64,127]$. At the other end of the complexity spectrum, perfect laminar or turbulent flows are the most convenient options.

Once the velocity field is characterized, mass transport can be investigated. The most accepted approximation for slurry reactors is a one phase system 
with high Pe numbers. Hence, the governing equation becomes represented by advection and homogeneous reaction only [128-130].

For immobilized systems it is easier to couple the reaction rate to mass transfer, given the clear definition for the interface. The most realistic transport models for the flow channel take into account both advection and diffusion [131-135]. All these papers neglect the internal mass transport inside the catalyst film. Hence, the reaction rate is set as the boundary condition for the catalyst-fluid interface. A more manageable method is to represent mass transport through the stagnant film model. The mass transfer coefficient can be determined experimentally with the benzoic acid dissolution method $[92,136]$ or can be computed via empirical correlations from Reynolds and Schmidt numbers [93, 137-139].

Microreactors are a special case. The modeling of such systems is straightforward due to their laminar flow and constant photon flux density throughout the entire surface of the reactor. Moreover, for immobilized catalyst, interface scattering due to roughness is neglected. Bulk scattering is not relevant due to absorption. Hence, the RTE simplifies to a Lambert-Beer law.

Very few papers seem to exploit the potential for accurate modeling of microreactors. Most of the articles employ the PFR equation [140-143]. Meng et al. [143] report values for reaction rate constants without providing an equation. It is assumed the same PFR equation is used due to its widespread use. Gorges et al. [140] validate the intrinsic character of $k$ by proving their system is placed in the reaction limiting regime. Lindstrom et al. [144] adopts the same approach of employing Damköhler number to assess mass transfer restrictions. However, for the apparent reaction rate constant they use the highest value in the literature, without computing the one for their system. Charles et al. [145] used the axially dispersed plug flow model to set up the mass balance in the microreactor. Mass transfer in the radial direction was represented by the stagnant film. Both the axial dispersion and mass transfer coefficients were computed from empirical relations. The kinetic model was initially Langmuir-Hinshelwood, but later on simplified to a first order-like equation. The kinetic coefficients were fitted to the model via iteration.

Nielsen et al. [65] derived a reaction rate expression based on semiconductor physics. The governing equation they set up for the catalyst layer comprised of diffusion and reaction. The gas concentration in the flow channel was considered constant by keeping the conversion under $10 \%$. They also managed to define a continuity equation for the localized excess hole concentration.

The current article draws attention on the simplicity of accurate modeling 
in the case of microreactors and proposes them as a pertinent choice when attempting to extract kinetic parameters. A complete model for immobilized photocatalytic microreactors is set up for the first time and solved for both light independency and light dependency first order kinetics. Experimental data obtained for various residence times, catalyst thicknesses and photon flux densities proves the model is capable of describing the reactor performance. Furthermore, a criterion is defined based on the absorption coefficient and catalyst thickness to mark the transition towards the regime where the incorporation of photon flux density is required. Performance parameters are also derived for the light dependent model for which the internal effectiveness factor reveals both mass transfer and light limitations.

The potential of the models is not confined to microreactor engineering. Scaleup is straightforward compared to slurry reactors as only channel height and catalyst thickness should be designed for a specific flowrate.

\subsection{Materials and Methods}

\subsubsection{Microreactor fabrication}

The silicon chips cut from $4^{\prime \prime}$ wafers were cleaned with $65 \%$ nitric acid (Merck) for $15 \mathrm{~min}$ and rinsed with water and acetone. Following the cleaning, the substrates were covered entirely with a commercial $\mathrm{TiO}_{2}$ suspension (VP Disp. W $2730 \mathrm{X}$, Evonik) and spin coated at $3000 \mathrm{rpm}$ angular velocity and 524 $\mathrm{rpm} / \mathrm{s}$ acceleration. The spin coating time was kept at $1 \mathrm{~min}$. The resulted layer was sintered for $2 \mathrm{~h}$ at $500{ }^{\circ} \mathrm{C}$ in air. The heating and cooling rates were kept at $2{ }^{\circ} \mathrm{C} / \mathrm{min}$.

The standard thickness obtained with the unprocessed dispersion of $30 \%$ (wt.) solid content was around $1200 \mathrm{~nm}$. Thinner layers were prepared by decreasing the viscosity of the dispersion. $310 \mathrm{~nm}$ and $640 \mathrm{~nm}$ were obtained by diluting the dispersion with distilled water to 15 and respectively $24 \%$ solid content. Thicker films were prepared by multiple coatings with sintering in between. The alteration of photocatalytic activity by repeated sintering was ruled out when the same degradation was observed for layers with one and two sintering cycles. As a general rule, the thickness of the catalyst layer was multiplied by the number of coatings. 
microstructured mold

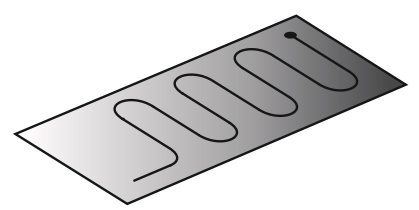

cast PDMS prepolymer

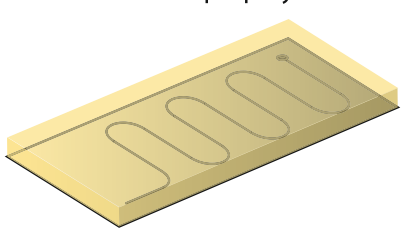

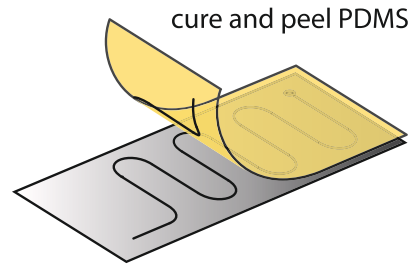
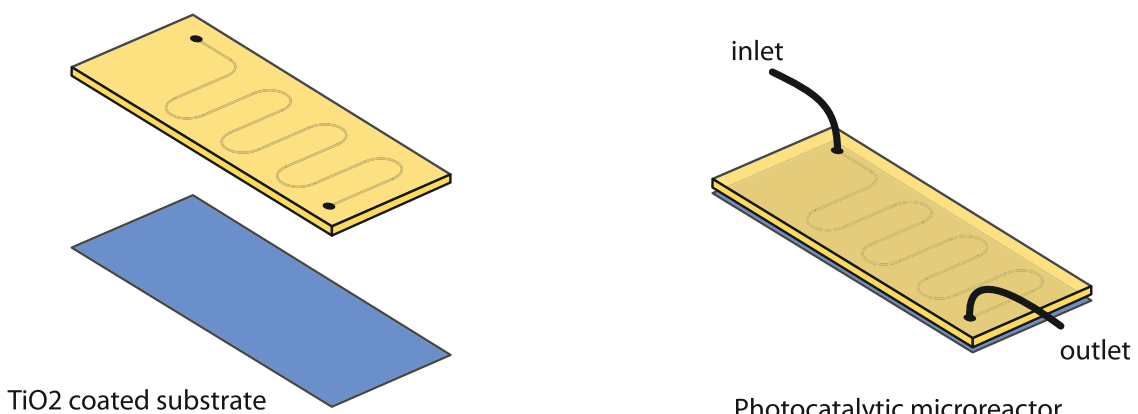

Photocatalytic microreactor

Figure 3.1: Schematic of the micro reactor fabrication steps. PDMS is cast on a microstructures mold and released after curing. The PDMS slab is subsequently sealed to a $\mathrm{TiO}_{2}$ coated substrate.

Catalytic films of 310, 640, 1200, 2050, 3000 and $4000 \mathrm{~nm}$ were used for degradation experiments.

The microreactor was assembled by attaching the $\mathrm{TiO}_{2}$ coated substrate to a PDMS slab containing the microchannel replicated from a microstructured mold. The PDMS was prepared by blending the polymer base (RTV-615 A, Permacol) with the curing agent (RTV-615 B, Permacol) in 10:1 ratio as indicated by the manufacturer. The mixture was poured on top of a patterned wafer and degassed in a vacuum desiccator. After partial curing for $45 \mathrm{~min}$ at $60{ }^{\circ} \mathrm{C}$, while still preserving its adhesive properties, the PDMS slabs were cut out from the mold, punctured for the inlet and outlet connections, attached to the substrate containing the catalyst layer and cured for another $45 \mathrm{~min}$. To ensure a leakfree assembly, additional PDMS was poured and cured for $3 \mathrm{~h}$ at $70^{\circ} \mathrm{C}$. The inlet and outlet connections were made by introducing needles into the previously punctured holes. The preparation steps are illustrated in Fig. 3.1.

The microstructured mold was made by standard photolithography using neg- 


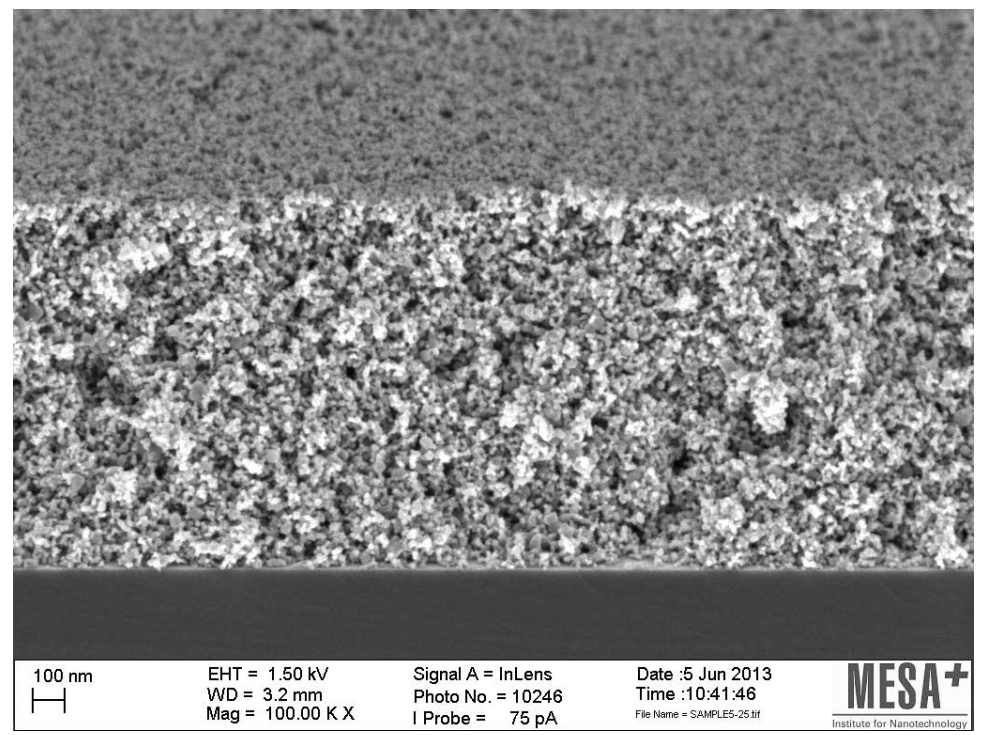

Figure 3.2: SEM cross-section of a porous $\mathrm{TiO}_{2}$ film.

ative photoresist (SU-8). SU-8 was spun on silicon wafer at $500 \mathrm{rpm}$ for 10 seconds and continued to $100 \mathrm{rpm}$ for 30 seconds. Then the sample was prebaked at $50,65,95{ }^{\circ} \mathrm{C}$ for $10,10,45$ minutes respectively. This is followed by UV exposure for 33 seconds and post baking at $50,65,80{ }^{\circ} \mathrm{C}$ for $5,10,20$ minutes respectively. Finally the sample was spray developed by RER 600 . The resulted SU-8 height was measured with optical profiler. The channel is rectangular and follows a meandering path. The basic dimensions are: $50 \mu \mathrm{m}$ height, $500 \mu \mathrm{m}$ width and $5.96 \mathrm{~cm}$ length, with a total volume of $1.49 \mu \mathrm{l}$.

\subsubsection{Catalyst layer characterization}

High resolution SEM showed a highly homogeneous surface with a narrow particle size distribution around $20 \mathrm{~nm}$. The cross-section (Fig. 3.2) shows also a uniform porosity throughout the layer.

M2000-X variable angle spectroscopic ellipsometer by J.A. Woollam was used for fast and non-invasive thickness measurements. The accuracy of the method was validated by comparing one of the results with the value obtained from SEM. Porosity and roughness could also be extracted by ellipsometry measurements. Porosity was determined by the Bruggeman Effective Medium 


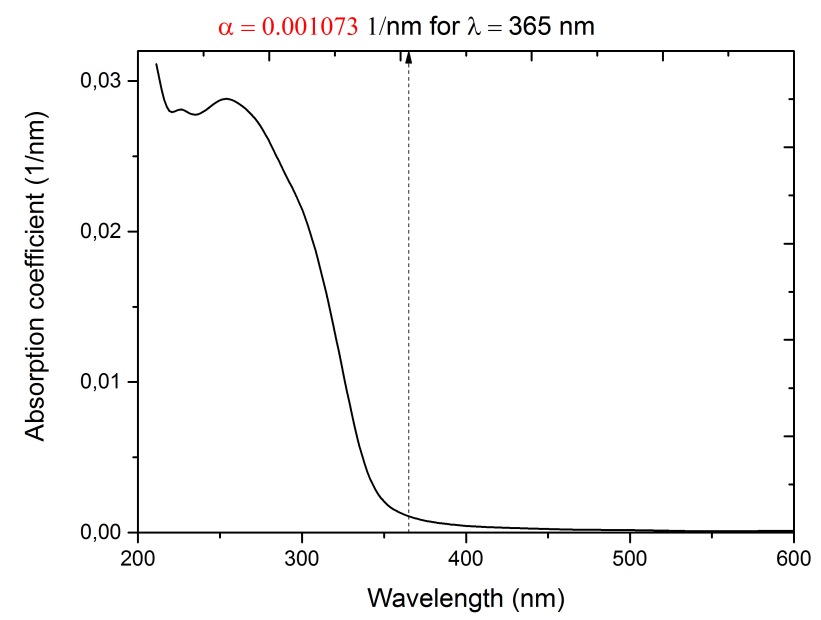

Figure 3.3: Spectral absorption coefficient of a $\mathrm{TiO}_{2}$ layer.

Approximation, EMA, [146] by mixing properties of dense $\mathrm{TiO}_{2}$ and void in a self consistent manner. Bruggeman type of EMA was used because it is well valid when the volume fractions of both components are comparable. Roughness was also estimated using EMA, this time adding a layer on top of the samples with assumed $50 \%$ of material and $50 \%$ of void and fitting its thickness. Values of $45 \%$ and $10 \mathrm{~nm}$, respectively, were consistent for thicknesses ranging between 300 and $4000 \mathrm{~nm}$. The morphology of layers is reproducible due to the same spin coating parameters used during preparation.

Ellipsometry could also be used for evaluating optical constants. Due to the homogeneity of the film, scattering was almost absent. Consequently, the model fitted very well and the accuracy of the measurement was high. For the extinction coefficient determination, we modeled the dielectric dispersion of the material by parametrization with B-Splines which is basically a polynomial spline function [147]. It works well when normal parametrization of the dielectric function requires too many dielectric oscillators. We also forced the dispersion to be Kramers-Kronig consistent, namely physically viable.

The extinction coefficient can be easily converted to absorption coefficient (Fig. 3.3) using the formula: $\alpha=4 \pi \beta / \lambda$ where $\beta$ is the extinction coefficient, $\alpha$ is the absorption coefficient $(1 / \mathrm{nm})$ and $\lambda$ is the wavelength $(\mathrm{nm})$. The method was validated by comparing the results with the absorption coefficient computed from transmission measurements of $\mathrm{TiO}_{2}$ films of varying thickness 


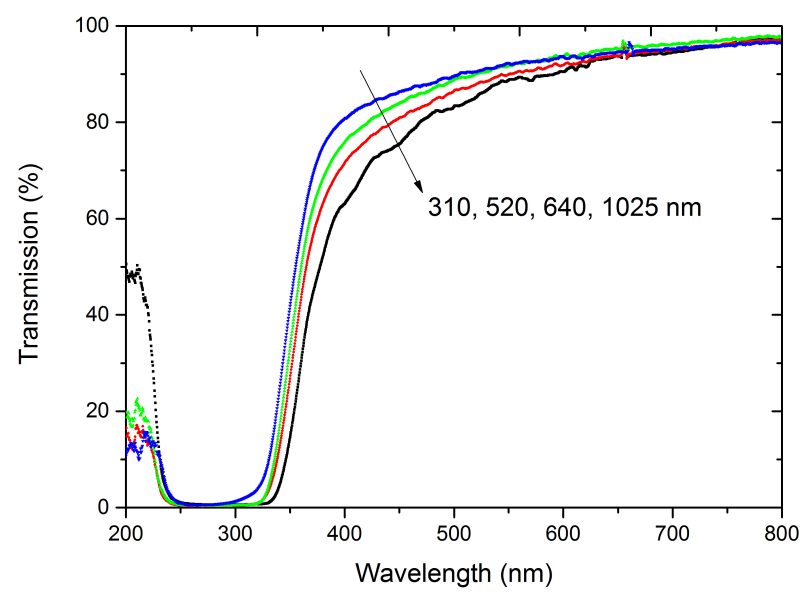

Figure 3.4: Transmission spectra for multiple catalyst thicknesses

(Fig. 3.4). The transmission measurements were performed by connecting a FHS-UV In-Line Filter Holder by Ocean Optics to the UV-VIS spectrometer (USB2000+ Miniature Fiber Optic Spectrometer by Ocean Optics) where the substrate coated with the catalyst film was fixed. Quartz glass was used as a substrate due to its transparency in the far UV range. Cleaned quartz glass was used to reference the transmission. The absorption coefficient was computed only for a single wavelength as a mere validation tool from the slope of the straight line fitting $-\ln (T / 100)$ against thickness based on the Lambert-Beer law: $T=I / I_{0} \cdot 100=e^{-\alpha \Delta x}$

\subsubsection{Microreactor operation}

An aqueous solution of $10 \mathrm{mg} / \mathrm{L}$ cortisone acetate (CA) (Sigma-Aldrich) was injected into the microreactor with flowrates of $5,10,20,30,40$ and $50 \mu \mathrm{L} / \mathrm{min}$ using a syringe pump.

The CA concentration was determined by light absorbance measurements using USB2000+ Miniature Fiber Optic Spectrometer by Ocean Optics. The monitored wavelength was $244.48 \mathrm{~nm}$, corresponding to the maximum absorption peak of CA. The calibration was carried out for different concentrations of CA solutions (from 5 to $20 \mathrm{mg} / \mathrm{L}$ ). 
When adsorption reached steady state and the concentration recorded by the UV-VIS spectrometer showed the inlet value, the UV lamp (UV-Point Source HP-120, Dr. Gröbel UV-Electronik GmbH) was turned on. The photocatalytic degradation of CA was continuously monitored by passing the outlet stream through a flowcell connected to the spectrometer. For the first batch of experiments, the irradiance was set at $180 \mathrm{~W} / \mathrm{m}^{2}$. For the second part, the light intensity was varied between 55 and $270 \mathrm{~W} / \mathrm{m}^{2}$ by changing the distance between the light guide and the microreactor. Due to the sharp emission peak, there was no necessity for integrating the irradiance over the whole $\mathrm{TiO}_{2}$ absorbing wavelength range. The total incoming photon flux density was approximated to the value corresponding to $365 \mathrm{~nm}$ wavelength which was measured using an optical power meter (Newport 1916-R).

$\mathrm{O}_{2}$ was continuously replenished through the permeable PDMS layer. The $\mathrm{O}_{2}$ permeability of the PDMS layer was tested by monitoring the $\mathrm{O}_{2}$ concentration after passing $\mathrm{O}_{2}$ depleted water through the reactor. The $\mathrm{O}_{2}$ inline detector recorded a steady state concentration very close to the maximum solubility of $\mathrm{O}_{2}$ in water at atmospheric pressure.

\subsection{Model}

\subsubsection{Light independent model}

Fig. 3.5 illustrates the schematics of the model. The reactor was divided in 2 domains: the flow channel and the catalyst film.

The governing equation in the catalyst layer includes diffusion and reaction only. The reaction rate is assumed to be first order with respect to the cortisone acetate, zero order with respect to oxygen due its provision through the permeable PDMS layer and zero order with respect to irradiance. Hence, the mass balance for the catalyst layer reduces to:

$$
D_{e f f} \frac{\partial^{2} c}{\partial y^{2}}-k c=0
$$

Where $D_{\text {eff }}=D \cdot \frac{\epsilon}{\tau}$ is the effective diffusion coefficient, $D$ is the molecular diffusion coefficient for cortisone acetate in water, $\epsilon$ is the porosity, $\tau$ is the tortuosity and $k$ is the first order reaction rate constant. 


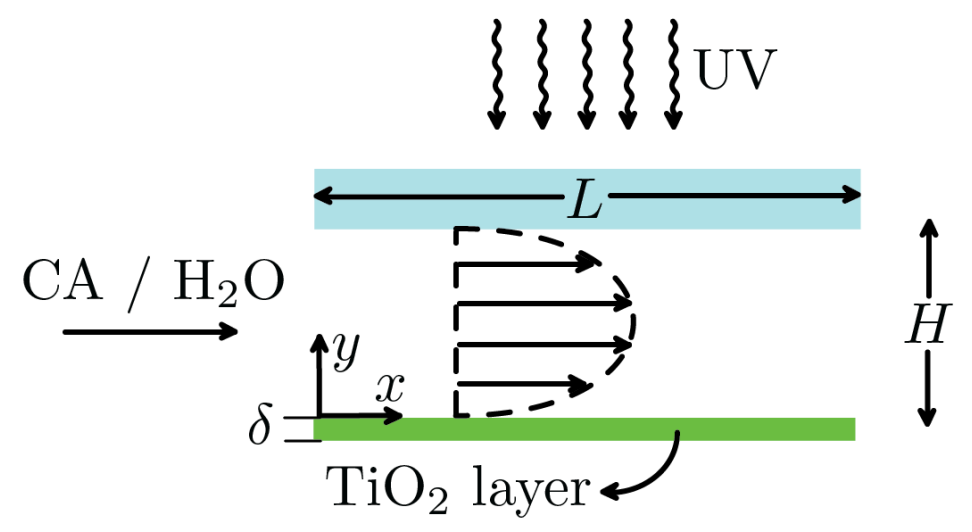

Figure 3.5: Schematic representation of the 2D model, indicating channel length $L$, channel height $H$, and catalyst thickness $\delta$

The bottom boundary for the catalyst layer has the flux equal to zero:

$$
y=-\delta: D_{e f f} \frac{\partial c}{\partial y}=0
$$

The boundary condition at the interface between the channel and the catalyst is represented by flux and concentration continuity:

$$
y=0: c=c_{L}, D_{e f f} \cdot \frac{\partial c}{\partial y}=D \frac{\partial c_{L}}{\partial y}
$$

The diffusion reaction equation together with the boundaries mentioned above have the following analytical solution:

$$
c(y)=c_{L} \cdot \frac{\cosh \left(\phi \cdot\left(1+\frac{y}{\delta}\right)\right)}{\cosh (\phi)}
$$

where Thiele modulus is $\phi=\sqrt{\frac{k}{D_{e f f}}} \cdot \delta$.

Hence, the domain of the catalyst layer can be collapsed into a single flux boundary condition for the flow channel:

$$
N_{y=0}=D_{e f f} \cdot \frac{\partial c}{\partial y}=\frac{D_{e f f} \cdot \phi \cdot c_{L}}{\delta} \cdot \tanh (\phi)
$$

The transport of the model compound in the flow channel is governed by advection and diffusion. 


$$
u(y) \cdot \frac{\partial c_{L}}{\partial x}=D \cdot \frac{\partial^{2} c_{L}}{\partial y^{2}}
$$

where $u(y)=u_{\text {avg }} \cdot\left(-6 \frac{y^{2}}{H^{2}}+6 \frac{y}{H}\right)$ represents a parabolic velocity profile.

The governing equation for the flow channel was non-dimensionalized to a square $1 \mathrm{x} 1$ domain (eq. 3.7) together with the boundary conditions (eq. 3.8 and 3.9) and numerically solved in Matlab.

$$
u_{\text {avg }} \cdot\left(-6 y^{\prime 2}+6 y^{\prime}\right) \cdot \frac{1}{L} \cdot \frac{H^{2}}{D} \cdot \frac{\partial c^{\prime}}{\partial x^{\prime}}=\frac{\partial^{2} c^{\prime}}{\partial y^{\prime 2}}
$$

where $x^{\prime}=x / L, y^{\prime}=y / H, c^{\prime}=c_{L} / c_{0}$.

$$
N_{y^{\prime}=0}=\frac{D_{e f f} \cdot \phi \cdot c^{\prime} \cdot H}{\delta} \cdot \tanh (\phi)
$$

for the boundary to the catalytic coating.

$$
N_{y^{\prime}=1}=D \frac{\partial c^{\prime}}{\partial y^{\prime}}=0
$$

for the upper wall.

\subsubsection{Light dependent model}

To account for light dependent conversion, the photon flux density was incorporated in the reaction rate described by photon absorption carrier generation first order kinetics. The expression was taken form Nielsen et al. [65], derived based on semiconductor physics. The governing equation for the catalyst layer becomes:

$$
D_{e f f} \frac{\partial^{2} c}{\partial y^{2}}-k c\left(\frac{\alpha \Phi_{0}}{B p_{0} n_{0} \hbar \omega}\right)^{\gamma} \exp (-\gamma \alpha y)=0
$$

where $B p_{0} n_{0} \cong 3.3 \cdot 10^{-23} \mathrm{~m}^{-3} \mathrm{~s}^{-1}$ is the equilibrium electron-hole recombination rate [65], $\Phi_{0}$ is the incoming photon flux density, $\hbar \omega$ is the photon energy, $\gamma$ is the transfer coefficient for the electron transfer process and $\alpha$ is the absorption coefficient.

Given that the boundary conditions remain the same, the new analytical solution for the concentration profile in the catalyst layer becomes: 


$$
c(y)=c_{L} \cdot \frac{I 1\left(\frac{2 \phi \sqrt{e^{\gamma \alpha \delta}}}{\gamma \alpha \delta}\right) \cdot K 0\left(\frac{2 \phi \sqrt{e^{-\gamma \alpha y}}}{\gamma \alpha \delta}\right)+K 1\left(\frac{2 \phi \sqrt{e^{\gamma \alpha \delta}}}{\gamma \alpha \delta}\right) \cdot I 0\left(\frac{2 \phi \sqrt{e^{-\gamma \alpha y}}}{\gamma \alpha \delta}\right)}{I 0\left(\frac{2 \phi}{\gamma \alpha \delta}\right) \cdot K 1\left(\frac{2 \phi \sqrt{e^{\gamma \alpha \delta}}}{\gamma \alpha \delta}\right)+K 0\left(\frac{2 \phi}{\gamma \alpha \delta}\right) \cdot I 1\left(\frac{2 \phi \sqrt{e^{\gamma \alpha \delta}}}{\gamma \alpha \delta}\right)}
$$

where $\phi^{2}=\frac{k_{i} \cdot \delta^{2}}{D_{\text {eff }}} \cdot\left(\frac{\alpha \Phi_{0}}{B p_{0} n_{0} \hbar \omega}\right)^{\gamma}$ and $\mathrm{I}_{n}(\mathrm{x})$ and $\mathrm{K}_{n}(\mathrm{x})$ are modified Bessel functions of the first and second kind.

This translates in the following boundary condition for the flow channel, giving the flux towards the catalyst layer which is again non-dimensionalized and implemented in Matlab:

$$
N_{y^{\prime}=0}=\frac{D_{e f f} \cdot \phi \cdot H \cdot c^{\prime}}{\delta} \cdot \frac{I 1\left(\frac{2 \phi \sqrt{e^{\gamma \alpha \delta}}}{\gamma \alpha \delta}\right) \cdot K 1\left(\frac{2 \phi}{\gamma \alpha \delta}\right)-K 1\left(\frac{2 \phi \sqrt{e^{\gamma \alpha \delta}}}{\gamma \alpha \delta}\right) \cdot I 1\left(\frac{2 \phi}{\gamma \alpha \delta}\right)}{I 0\left(\frac{2 \phi}{\gamma \alpha \delta}\right) \cdot K 1\left(\frac{2 \phi \sqrt{e^{\gamma \alpha \delta}}}{\gamma \alpha \delta}\right)+K 0\left(\frac{2 \phi}{\gamma \alpha \delta}\right) \cdot I 1\left(\frac{2 \phi \sqrt{e^{\gamma \alpha \delta}}}{\gamma \alpha \delta}\right)}
$$

Note that the governing equation is the same for both the light dependent and light independent model, and that the two models only differ in the boundary condition describing the flux into the catalyst layer.

The geometry of the channel was simplified to a rectangle preserving the height, $H$, of $50 \mu \mathrm{m}$ and the length, $L$, of $5.96 \mathrm{~cm}$. The mesh was refined such that mesh independent results were obtained.

\subsection{Results and discussion}

\subsubsection{Light independent model (LIM)}

The assumption for a first order reaction rate was first justified by evaluating the effect of different residence times ranging between 1.8 and $18 \mathrm{~s}$. The degradation vs. residence time curve shown in Fig. 3.6 corresponds to $2050 \mathrm{~nm}$ catalyst thickness in order to capture the complete spectrum for the residence time range used during the experiments.

$k=31$ 1/s was extracted by fitting the model for the lowest residence time where experimental data is more reliable. The reaction rate constant values determined for various catalyst thicknesses ranged between 21 and 43 1/s. Their 


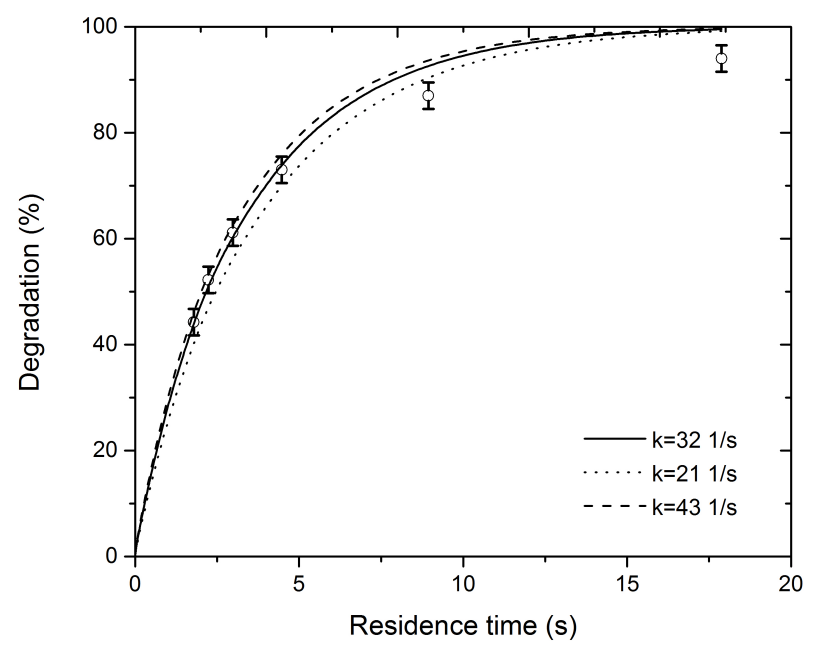

Figure 3.6: Degradation of Cortisone acetate (CA) vs. residence time for 2050 $\mathrm{nm}$ catalyst layer

corresponding degradation curves were also plotted in Fig. 3.6. The standard deviation for the degradation is around $\pm 2.5 \%$. It was assessed based on the systematic deviation of the base line during the absorption measurements.

The consistency of $k$ is set out in Fig. 3.7 where degradation is plotted against catalyst thickness ranging between $300 \mathrm{~nm}$ and $4000 \mathrm{~nm}$ for multiple residence times. An optimum catalyst thickness can already be deduced given the fact that an increase in thickness beyond $2000 \mathrm{~nm}$ affects the degradation only slightly.

The intrinsic $k$ values are several orders of magnitude higher than the apparent first order reaction rate constants reported in the literature. For example, for methylene blue (MB), a common model compound, values between $10^{-5}$ and $10^{-2} 1 / \mathrm{s}$ are usually assigned $[98,105,142,143,148-158]$. The values increase for smaller reactor scales, reaching approximately $0.31 / \mathrm{s}$ for the present study. Following the same experimental routine and simulation procedure, given the molecular diffusion coefficient for MB in water, $D=5.7 \cdot 10^{-10} \mathrm{~m}^{2} / \mathrm{s}$, a value of $k=401 / \mathrm{s}$ was fitted. The difference of two orders of magnitude between the real and apparent reaction rate constant draws attention on the importance of accurate modeling. The intrinsic value for the reaction rate constant allows 


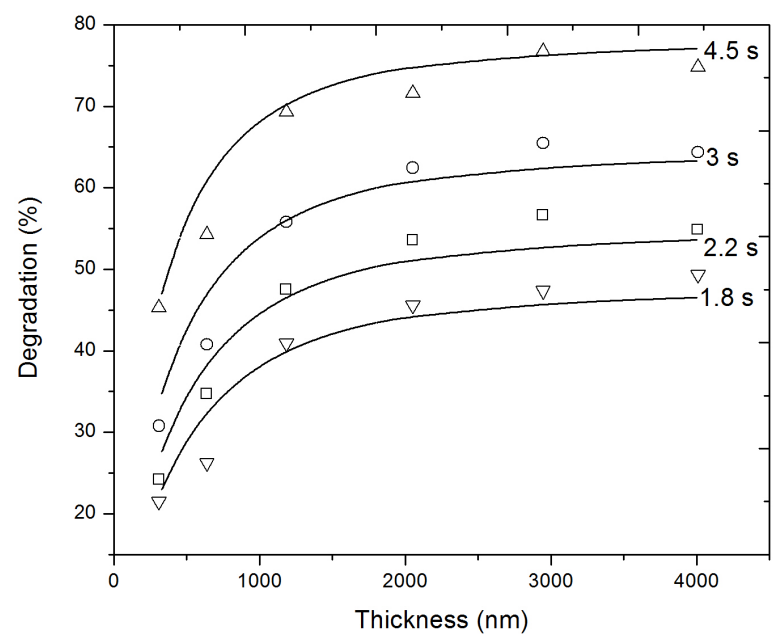

Figure 3.7: Degradation vs. thickness for multiple residence times. Symbols depict experimental results and lines correspond to the light independent model with $k=321 / \mathrm{s}$

catalyst comparison, as well as the evaluation of mass transfer for different reactor configurations.

The use for the extracted reaction rate constant can also be extended to different catalyst densities or dispersed systems by expressing it per unit surface area. The conversion from $k$ per unit volume $[1 / \mathrm{s}]$ to $k^{\prime \prime}$ per units surface area $[\mathrm{m} / \mathrm{s}]$ has the known formula: $k^{\prime \prime}=\frac{k}{S_{a} \rho_{c}(1-\epsilon)}$, where $S_{a}=9 \cdot 10^{4} \mathrm{~m}^{2} / \mathrm{kg}$ is the specific surface area, $\rho_{c}=3895 \mathrm{~kg} / \mathrm{m}^{3}$ is the anatase density and $\epsilon=0.45$ is the porosity. Consequently, MB has $k^{\prime \prime}=2.07 \cdot 10^{-7} \mathrm{~m} / \mathrm{s}$ corresponding to $k=401 / \mathrm{s}$, while CA has $k^{\prime \prime}=1.66 \cdot 10^{-7} \mathrm{~m} / \mathrm{s}$ corresponding to $k=321 / \mathrm{s}$. Not to forget, the use of light independent kinetics is valid when the reaction rate can be volume averaged with respect to the field of local volumetric rate of photon absorption. The case of immobilized systems will be discussed later by comparing the light independent with the light dependent model.

For a quantitative optimization, the diffusion/reaction limiting regimes were investigated by evaluating the effect of $k$ on degradation, in terms of Thiele modulus. For a first order reaction occurring in a planar porous catalyst, the Thiele modulus can be computed using the following formula: $\phi=\sqrt{\frac{k}{D_{e f f}}} \cdot \delta$. 


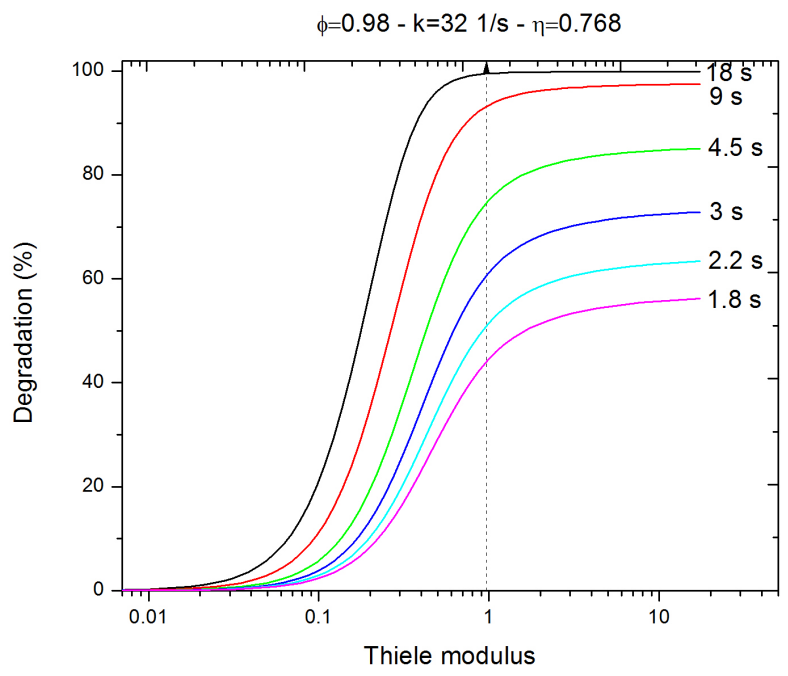

Figure 3.8: Degradation by $2050 \mathrm{~nm}$ catalyst layer vs. Thiele modulus for multiple residence times

The overall degradation given by a $2050 \mathrm{~nm}$ catalyst film was plotted in Fig. 3.8 against Thiele modulus for $k$ ranging between $10^{-3}$ and $10^{4} 1 / \mathrm{s}$.

As $k$ and, consequently, Thiele modulus start to increase, the intrinsic chemical reaction rate starts to compete with the given diffusion rate. For the experimentally determined kinetics, $k=321 / \mathrm{s}, D_{\text {eff }}=1.4 \cdot 10^{-10} \mathrm{~m}^{2} / \mathrm{s}$ and $\delta=2050 \mathrm{~nm}$, Thiele modulus is 0.98 .

We will later analyze the effectiveness of the catalyst according to this model, combined with the light dependent model.

\subsubsection{Light dependent model (LDM)}

An important part in designing a photocatalytic reactor is related to light intensity distribution as effective use of light has a big impact on the economics of the process.

Whenever kinetics are modeled with respect to light intensity, $I$, the reaction rate is considered to follow a power law: $r=b I^{\gamma}$. Based on semiconductor physics, the physical meaning of $\gamma$ would be the transfer coefficient of the 


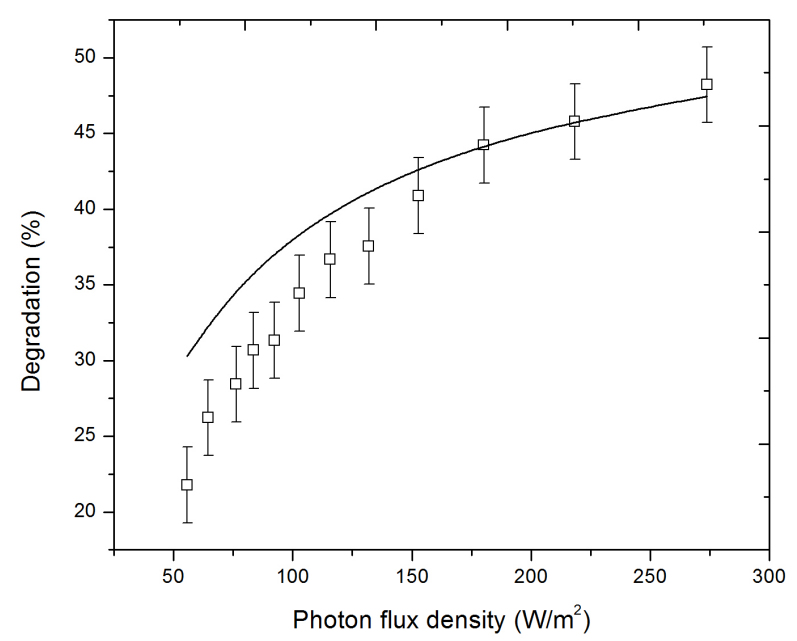

Figure 3.9: Degradation by $2050 \mathrm{~nm}$ catalyst layer vs. photon flux density. $k_{i}=6.46 \cdot 10^{-48} 1 / \mathrm{s}, \alpha=1.073 \cdot 10^{6} 1 / \mathrm{m}, \gamma=1$

electron transfer process. There is a general misunderstanding about $\gamma$, which is usually confused with the apparent $\gamma$ extracted by fitting the conversion against the irradiance in a power law fit.

The real decrease in $\gamma$ given by the higher enhancement in the recombination rate in connection with the excess hole production rate was found to happen at light intensities higher than $250 \mathrm{~W} / \mathrm{m}^{2}$ [159]. Moreover, values lower than 1 would translate in decreased efficiency regarding the use of light, rendering this operating range with less practical value.

The model was evaluated for the effect of different incoming photon flux densities. Fig. 3.9 shows the degradation by the $2050 \mathrm{~nm}$ catalyst film plotted against irradiance for values ranging between 55 and $270 \mathrm{~W} / \mathrm{m}^{2}$.

For the light dependent reaction rate, $r=k_{i}\left(\frac{\alpha \Phi_{0}}{B p_{0} n_{0} \hbar \omega}\right)^{\gamma} \exp (-\gamma \alpha y) \cdot c$, the constant $k_{i}$ was extracted by fitting the model to the experimental data for the higher light intensities. In this range, degradation is almost independent on the light intensity. The small order of magnitude $k_{i} \cong 10^{-48} 1 / \mathrm{s}$ comes from the high value of the prefactor $\left(\frac{\alpha \Phi_{0}}{B p_{0} n_{0} \hbar \omega}\right)^{\gamma} \cong 10^{49}$.

The challenge with the incorporation of light into the kinetic model does not come only from the increased difficulty in mathematical manipulation. The 


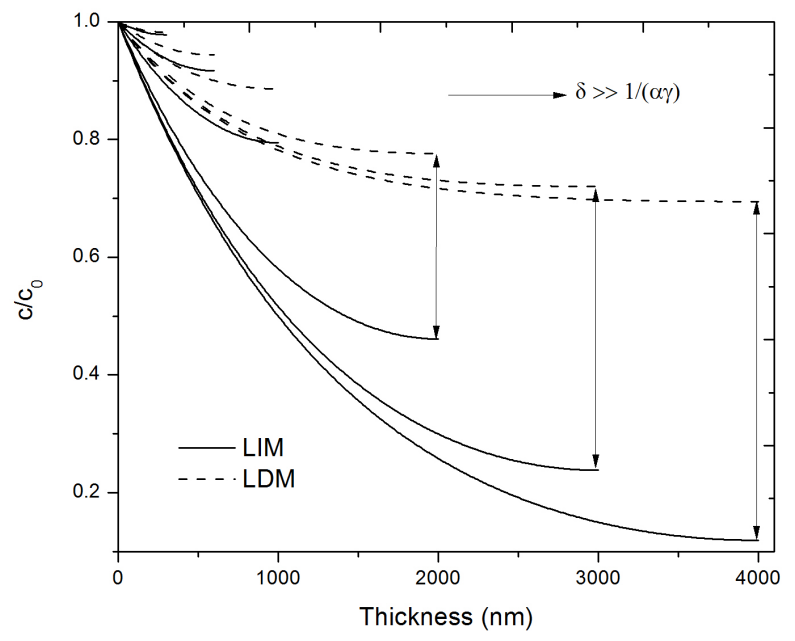

Figure 3.10: Normalized concentration profiles vs. catalyst thickness. $k=$ $69.4851 / \mathrm{s}$ for LIM, $k_{i}=6.46 \cdot 10^{-48} 1 / \mathrm{s}, \alpha=1.073 \cdot 10^{6} 1 / \mathrm{m}, \gamma=1, \Phi_{0}=180$ $\mathrm{W} / \mathrm{m}^{2}$ for $\mathrm{LDM}$

most important disadvantage is the high number of unknowns that come into play when attempting to understand the photon absorption carrier generation mechanism. Simple engineering tools are very important for reactor design and optimization. A simplistic model could pave the way towards a consensus regarding photocatalytic reactor design and optimization and speed up the industrial implementation. Hence, a thorough comparison between the two models was performed in order to identify the regime where the simplistic model is an admissible approximation.

\subsubsection{Criteria for neglecting light intensity}

Previously, $k$ was averaged for multiple catalyst thicknesses. However, when light is modeled, $k$ is no longer constant throughout the thickness and follows an exponential decay: $k(y)=k_{i}\left(\frac{\alpha \Phi_{0}}{B p_{0} n_{0} \hbar \omega}\right)^{\gamma} \exp (-\gamma \alpha y)$. We can define a characteristic decay length: $\delta=1 / \gamma \cdot \alpha=932.3 \mathrm{~nm}$ where the normalized $k(y), k^{\prime}(y)=\exp (-\gamma \alpha y)$ decreases to $1 / e$ of its initial value. This light decay 
length (LDL) is very useful in order to point out the transition towards the regime where light incorporation is recommended.

The significance of LDL can be visualized by plotting the normalized concentration profiles for both models shown in Fig. 3.10. To have a common starting point $k_{L I M}$ was equalized to $k_{y=0}=k_{i} \cdot\left(\frac{\alpha \Phi_{0}}{B p_{0} n_{0} \hbar \omega}\right)^{\gamma}=69.4851 / \mathrm{s}$. For thicknesses higher than LDL, the concentration profiles of the two models become significantly distinct.

\subsubsection{Updated performance parameters}

A conclusive discussion has to include performance parameter investigation, as the efficient use of catalyst is crucial to the design procedure [160]. Given the first order reaction in the planar porous catalyst for the LIM, the internal effectiveness factor has the well known expression: $\eta=\frac{\tanh \phi}{\phi}$, where $\phi=\sqrt{\frac{k}{D_{e f f}}} \cdot \delta$. In the case of LDM, the expressions were derived based on definitions. The internal effectiveness factor represents the ratio between the actual reaction rate and the rate in the absence of concentration gradients. However, an easier way to find this is to use the flux at the surface of the catalyst which equals to the total reaction occurring inside. The internal effectiveness factor can then be expressed as:

$$
\eta=\frac{1}{\phi} \cdot \frac{I 1\left(\frac{2 \phi \sqrt{e^{\gamma \alpha \delta}}}{\gamma \alpha \delta}\right) \cdot K 1\left(\frac{2 \phi}{\gamma \alpha \delta}\right)-K 1\left(\frac{2 \phi \sqrt{e^{\gamma \alpha \delta}}}{\gamma \alpha \delta}\right) \cdot I 1\left(\frac{2 \phi}{\gamma \alpha \delta}\right)}{I 0\left(\frac{2 \phi}{\gamma \alpha \delta}\right) \cdot K 1\left(\frac{2 \phi \sqrt{e^{\gamma \alpha \delta}}}{\gamma \alpha \delta}\right)+K 0\left(\frac{2 \phi}{\gamma \alpha \delta}\right) \cdot I 1\left(\frac{2 \phi \sqrt{e^{\gamma \alpha \delta}}}{\gamma \alpha \delta}\right)}
$$

where the updated Thiele modulus was computed as the ratio between surface reaction rate and the diffusion rate:

$$
\phi^{2}=\frac{k_{i} \cdot \delta^{2}}{D_{\text {eff }}} \cdot\left(\frac{\alpha \Phi_{0}}{B p_{0} n_{0} \hbar \omega}\right)^{\gamma}
$$

The internal effectiveness factor is plotted for both models in Fig. 3.11. $\eta_{\text {LIM }}$ accounts only for mass transfer limitation, while $\eta_{L D M}$ considers both diffusion and radiative transport when assessing catalyst coverage. The relative effectiveness factor is an indication for which transport is limiting. While in the top layer light and consequently, reaction rate are controlling, when the relative effectiveness factor levels off, mass transfer takes over and no further improvement in conversion can be sought. It can be noticed that the thickness 


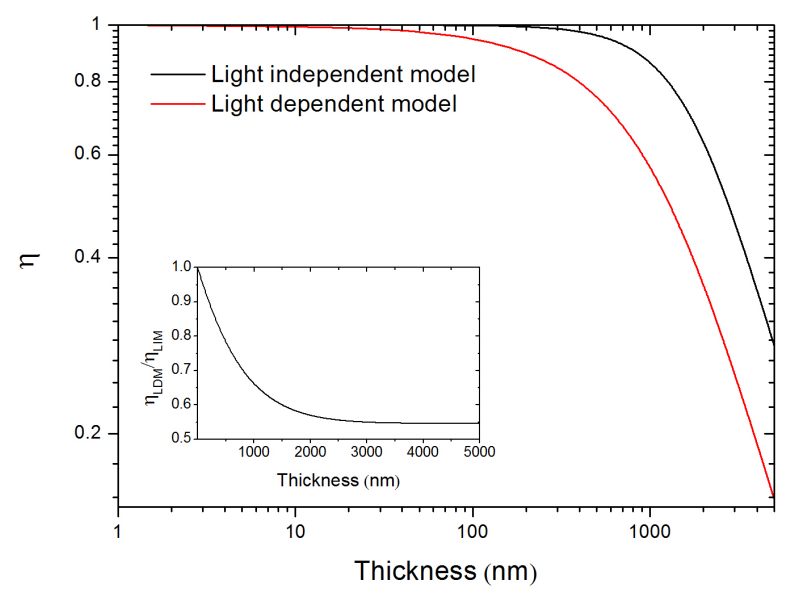

Figure 3.11: Internal effectiveness factor vs. catalyst thickness. $k=69.485$ $1 / \mathrm{s}$ for LIM, $k_{i}=6.46 \cdot 10^{-48} 1 / \mathrm{s}, \alpha=1.073 \cdot 10^{6} 1 / \mathrm{m}, \gamma=1, \Phi_{0}=180 \mathrm{~W} / \mathrm{m}^{2}$ for LDM. The insert shows the LDM/LIM effectiveness factor

corresponding to the transition between the two regimes is the same for which conversion reached its maximum in Fig. 3.7. Hence, the relative effectiveness factor can be considered the absolute performance parameter for immobilized photocatalytic reactors.

The maximum conversion thickness depends on the absorption coefficient and incoming photon flux density. For higher $\alpha$, the initial decrease becomes steeper, lowering the maximum conversion thickness. For higher light intensities, the maximum conversion thickness is also decreasing due to the higher $k_{y=0}$. The difference is in the asymptotic value for the relative effectiveness factor which suggests how far away the two models are from each other. For higher $\alpha$ the discrepancy between the two models is increasing, while for higher light intensities it is decreasing.

\subsection{Conclusions}

Accurate modeling for microreactors can be achieved by applying basic physical mechanisms. This leads to a rational reactor design and easy optimization. 
The present article attempts a complete model for immobilized photocatalytic microreactors and explores their potential as investigation tools for intrinsic kinetics.

Models capable of describing reactor performance were build for a first order reaction rate with either light independency or light dependency described by photon absorption carrier generation mechanism. The extracted reaction rate constant reveals the intrinsic kinetics as both external and internal mass transport are accounted for. For the first time $k$ values on the order of magnitude $10^{1} 1 / \mathrm{s}$ are reported.

The simplification to the light independent model is justified by defining a criterion for neglecting light intensity based on film thickness and absorption coefficient. Performance parameters are also derived for the situation when irradiance has to be considered. The updated internal effectiveness factor reveals both mass transfer and light limitations. 


\section{4 \\ Fructose dehydration to hydroxyl-methylfurfural in an immobilized catalytic microreactor ${ }^{\circ}$}

In this chapter we report a microfluidic platform that allows for high temperature, high pressure conversion with inline spectroscopic measurement for a fast and accurate determination of both reaction rate constant and activation energy. The dehydration of fructose to hydroxyl-methylfurfural has been performed in this immobilized microreactor with both dense zirconia and porous titania layers, as a starting point to probe the potential of abundant metal oxides.

${ }^{\circ}$ Submitted as: Aura Visan and Rob G. H. Lammertink, Fructose dehydration to hydroxylmethylfurfural in an immobilized catalytic microreactor (2019) 


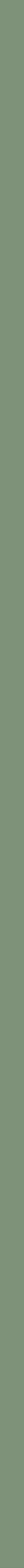




\subsection{Introduction}

Catalysis is ever-present in industry with up to $90 \%$ of the reaction processes using catalysts. However, this valuable experience covers mostly the fossil based feedstock processing which entails selective functionalization of apolar, unfunctionalized hydrocarbons. For well-known reasons, there is a global interest for more sustainable resources. The catalytic conversion of biomass waste is a promising alternative for chemicals, materials, and fuel production [161-163]. Especially for chemical synthesis, this route is challenging due to the richness and complexity of the chemical composition of biomass waste. While oil consists of hydrocarbons and, hence, is hydrophobic, biomass is high in oxygen content and consequently hydrophilic. Catalysts will have to fulfil completely different requirements in the case of biomass conversion, as the chemical conversion will entail selective defunctionalisation of polar, highly functionalized oxygenates.

Future bio-refineries need to produce high value biobased chemicals to be economically competitive. There are two possible strategies for approaching the market. The first is to aim for novel products with new and improved properties such is the case of 2,5-furandicarboxylic acid which has the prospect of replacing terephthalic acid in the fabrication of PET [164]. In this context, markets need to develop. The second scenario is to aim for existing products that utilize existing infrastructure and for which there is already a mature market [165]. Both processing routes converge towards a few important platform molecules from which a myriad of end products can diverge. Dusselier identified that carbohydrates give access to a plethora of novel and drop in chemicals. Some of these highly versatile chemicals include 5-hydroxymethylfurfural (HMF), levulinic acid (LA) and $\gamma$-valerolactone (GVL) [166-168].

HMF is a versatile and promising compound derived from carbohydrates. It can be used as a renewable intermediate for the production of polymers, fuels or solvents in the petrochemical industry. The last step in the synthesis of HMF is the dehydration of fructose (Figure 1). Researchers have developed biocatalysts to convert fructose from cellulose [169], which is one of the major component of most plants and agriculture wastes [170]. This is why an environmental-friendly process with a high fructose conversion rate is in strong demand for the overall conversion of biomass.

The challenge for the effective utilization of biomass is to develop novel and ecofriendly catalysts for efficient conversion at low cost [171-174]. Group VIII 
metals, notably ruthenium, have been shown to facilitate the hydrogenation step in liquefaction, but the cost of these precious metals is extremely high. Thus, inexpensive heterogeneous catalysts, such as zirconium dioxide and titanium dioxide are attracting increasingly more attention as alternatives [175]. Moreover, due to its high content in oxygen, biomass is typically processed in aqueous solutions or other polar solvents such as alcohols. These polar solvents at high temperatures and pressures and often at extreme $\mathrm{pH}$ challenge the stability of most catalysts. Among the few that can withstand these severe conditions are metals oxides.

Solid acids such as oxides [171,176,177], phosphates [178] and chlorides [179, 180], but also plain metal oxides $[176,181]$ have been studied as catalysts for the dehydration of fructose. It has been proven that metal oxides increase their acid site density upon treatment with phosphoric acid and thus improve the dehydration from fructose to HMF $[177,181]$. The treatment with phosphoric acid, esterifies $-\mathrm{OH}$ groups on the surface of $\mathrm{TiO}_{2}$ into $-\mathrm{O}-\mathrm{PO}\left(\mathrm{OH}_{2}\right)$ and increase the HMF selectivity $[171,177]$. What is more, $\mathrm{TiO}_{2}$ exhibits superhydrophilicity during and after UV light due to the formation of excess surface $-\mathrm{OH}$ groups under light irradiation. The combination of UV light and phosphoric acid treatment would increase the density of surface phosphate species, thereby enhancing its catalytic performance [171].

The optimization of catalyst design for biomass conversion is a prerequisite for making this technology industrially feasible. To assess these improvements, the activity investigation process has to be fast, restricted in energy and chemical use and, very importantly, decoupled from reactor design. Intrinsic kinetics can be retrieved when the system is accurately modelled. Microreactors have been proven to be pertinent tools for kinetic investigation. Their small dimensions provide a laminar flow profile and, consequently, a well-defined mass transport. They also allow for fast inline measurement which generates information in real time without the need to quench the reaction for sample collection. The current project investigates one of the key reactions in the conversion of biomass waste, namely fructose to hydroxyl-methylfurfural. 

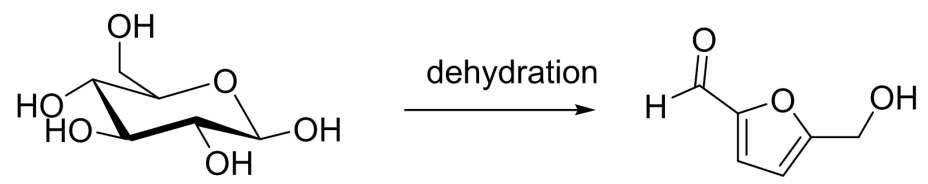

Figure 4.1: Reaction scheme showing the dehydration of fructose to hydroxylmethylfurfural

\subsection{Experimental}

\subsubsection{Chip fabrication}

The need for harsh conditions such as high temperature and high pressure requires a robust microreactor design. The fabrication of microchannels and inlet/outlet holes in silicon was achieved using photolithography and deep reactive ion etching. Anodic bonding of the glass cover to silicon gives a very strong bond without the need of excessive heating which could affect the morphology of the immobilized catalyst. Having the channels in silicon is beneficial due to its high thermal conductivity and glass is the obvious choice to close the microreactor because it gives the possibility of UV irradiation necessary for the surface functionalization of the catalyst and the use of microscopic techniques to evaluate in-situ humin deposition or bubble formation. The meandering channel (Figure 4.2) is $500 \mu \mathrm{m}$ wide and $50 \mu \mathrm{m}$ deep and $18 \mathrm{~cm}$ long.

We have used two types of catalysts. A dense zirconia layer which was sputtered during the cleanroom fabrication and a porous titania which was wash coated after closing the reactor. The cleanroom fabrication steps include photolithography, opening the $\mathrm{SiO}_{2}$ mask, deep reactive ion etching the microchannels, lift-off, second photolithography on the back, deep reactive ion etching the inlet and outlet holes, as well as the gap separating the heated area from the fluidic connections, again lift off, wet etching of the $\mathrm{SiO}_{2}$ mask, photolithography for the third time by spray coating the photoresist to homogeneously cover the 3D structures, sputtering the catalyst, lift off in acetone bath, anodic bonding and dicing. The success of this patterning is due to the thick resist that is obtained by spray coating. The $5 \mu \mathrm{m}$ layer ensures that no 
a.

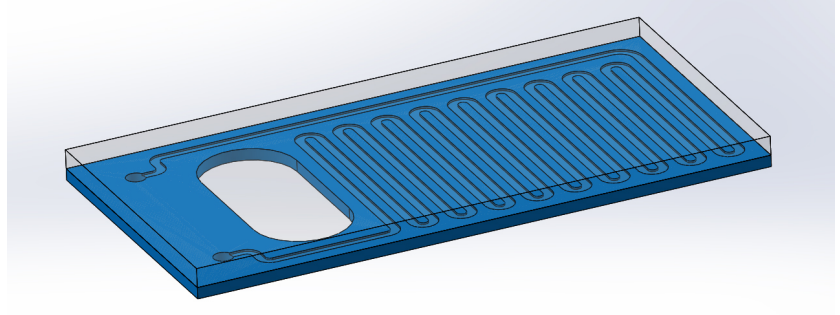

b.

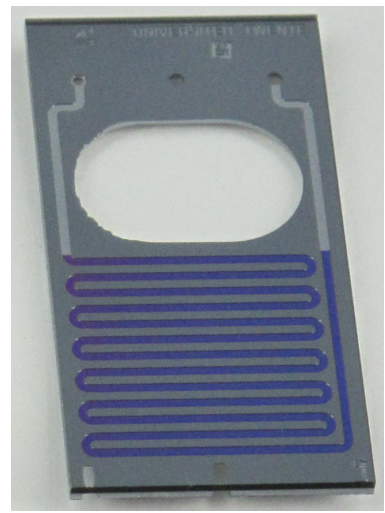

Figure 4.2: a. Microreactor CAD design b. The actual microreactor with the sputtered $\mathrm{ZrO}_{2}$ layer (visible in the purple colour)

edge connection takes place upon sputtering. The second type of microreactor does not require a third photolithography step and it was closed after deep reactive ion etching the back.

\subsubsection{Catalyst deposition and characterization}

Zirconium dioxide was deposited by reactive magnetron sputtering with a zirconium target using a dc power source. The film was sputtered for $40 \mathrm{~min}$ at $200 \mathrm{~W}$ using a reactor gas mixture of $92.5 \mathrm{vol} \% \mathrm{Ar}$ and $7.5 \mathrm{vol} \% \mathrm{O}_{2}$ at a process pressure of $5 \times 10^{-3} \mathrm{mbar}$. The layer was annealed for $4 \mathrm{~h}$ at $500{ }^{\circ} \mathrm{C}$ in air. The heating and cooling rates were kept at $2{ }^{\circ} \mathrm{C} / \mathrm{min}$. A thorough characterization of the layer was performed. High resolution scanning electron microscopy revealed a nonporous film with significant roughness. The elemental stoichiometry of the metal oxide was investigated using Energy Dispersive X-ray spectroscopy. The chemical composition was also confirmed by X-Ray Photoelectron Spectroscopy. The thickness and roughness could be determined by Spectroscopic Ellipsometry using the method described in Visan et al [67]. X-ray Diffraction was used to investigate the degree of crystallinity and detect the crystalline phases. The orientation of the crystallites was visualized by TEM.

The second microreactor was wash coated with a porous titania layer using 


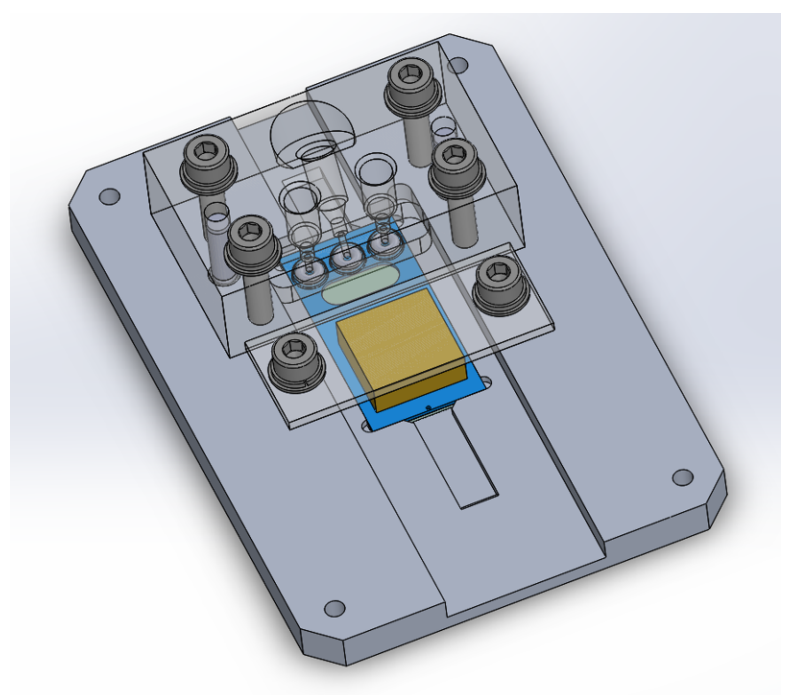

Figure 4.3: Chipholder CAD design displaying the configuration of the TEC element

a monodisperse commercial suspension (VP Disp. W 2730 X, Evonik). The suspension was used without dilution at the initial 30\% (wt.) solid content. The aqueous dispersion is pumped through the microchannel and flushed afterwards at a constant displacement velocity to ensure a constant thickness along the channel. The resulting layer was sintered for $2 \mathrm{~h}$ at $500{ }^{\circ} \mathrm{C}$ in air. The heating and cooling rates were kept at $2{ }^{\circ} \mathrm{C} / \mathrm{min}$. The quality of the suspension gave a highly homogeneous layer which was visualized using high resolution scanning electron microscopy. The narrow size distribution of the starting suspension was confirmed by light scattering measurements using a Zetasizer. The roughness and porosity could be determined by spectroscopic ellipsometry [67]. The crystalline composition is provided by the manufacturer.

After sintering the wash coated layer, $\mathrm{TiO}_{2}$ was treated with phosphoric acid under UV. The microchannels were flushed with $1 \mathrm{M} \mathrm{H}_{3} \mathrm{PO}_{4}$ solution for 15 min at $50 \mu \mathrm{l} / \mathrm{min}$. While filled with $\mathrm{H}_{3} \mathrm{PO}_{4}$ solution, the microreactors were fixed under UV light (Dr. Gröbel UV light source HP-120, $180 \mathrm{~mW} / \mathrm{cm}^{2}$ ) for 4 hours. After treatment, the microchannels were rinsed thoroughly with distilled water. 


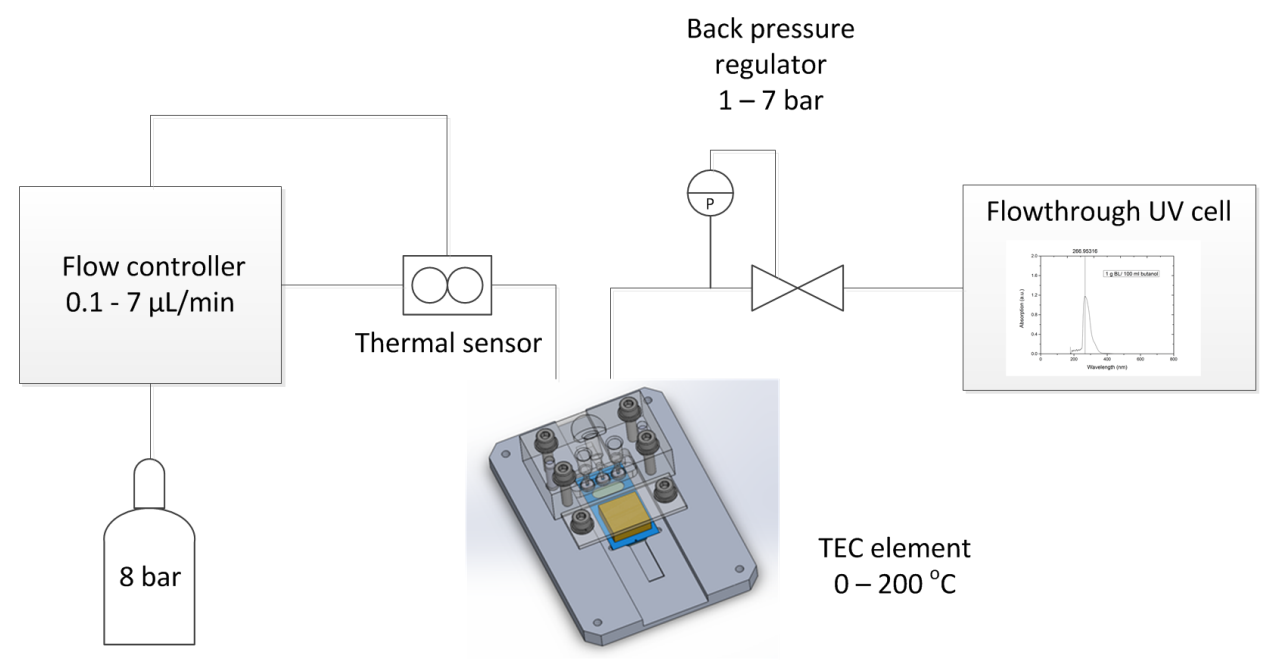

Figure 4.4: Schematic illustration of the used setup, combining temperature and flow control with inline UV-Vis analysis.

\subsubsection{Modular packaging}

We opted for modular packaging due to the plug and play feature (Figure 4.3). To achieve fluidic connection, compression needs to be used which requires orings. The stability of the o-rings is challenged for this combination of high temperature and pressure. A solution was found in the literature where the heated reaction area is separated from the connection side. We placed the heating element inside the chipholder to keep a compact design. To limit the heating at the connection side, a part of the silicon between the connection zone and heated zone is removed according to the design of Samuel Marre [182]. The temperature difference between the reaction zone and the port side has been assessed by using a second temperature sensor in order to check if active cooling is required on the compression side. The system proved to be leakage free until 50 bar. We opted for silicone o-rings due to their higher flexibility compared to Karlez. Karlez and Valco give good sealing only for the first use. The heating was performed locally on the reaction side using a Peltier element which allowed for accurate temperature control up to $200^{\circ} \mathrm{C}$. The chipholder has two separate top parts, the fluidic connection and the $\mathrm{Al}$ plate that pushes 
down the Peltier element which also works as a heat sink to ensure the heat flux through the element. This separate top prevents heat dissipation to the rest of the chipholder. The commercial temperature control system does not go higher than $120-150{ }^{\circ} \mathrm{C}$ for thermoelectric (TEC) cooling elements, so individual components were put together in house. Two separate temperature measurements are possible. On the reaction side this is done with a Pt100 sensor that provides a very high accuracy in the order of $10^{-3}{ }^{\circ} \mathrm{C}$, while a NPT sensor monitors the compression side. The thermocouple tip is positioned very close to the microchannels inside a separate pocket. A 24 watt resistive heater can replace the Peltier within the same control unit to extend the temperature range up to $250^{\circ} \mathrm{C}$. The chipholder was fabricated entirely from PEEK in order to minimize the heat loss from the Peltier element. It also has a window on the glass side of the chip which allows microscopic visualization.

\subsubsection{Setup and operation}

The setup allows to independently vary residence time, pressure and temperature and monitor conversion using inline analysis. The liquid handling system comprises a Fluigent flow controller equipped with a thermal sensor that is connected to a control unit which actively regulates the gas pressure necessary to achieve the flowrate set point. A back-pressure regulator using an active valve controls the pressure, decoupling in this way the flowrate from the pressure. Figure 4.4 shows the schematic of the set up used. After the pressure reaches the set point, the microreactor was heated to the desired temperature. The operating conditions for the showcase reaction, namely the conversion of fructose to hydroxymethylfurfural (HMF) are 7 bar and $130{ }^{\circ} \mathrm{C}$. The conversion is quantified by measuring the product concentration via inline UV-Vis spectroscopy that tracks the absorption in the UV range of HMF. The maximum absorption peak of HMF is at $284 \mathrm{~nm}$. The calibration was carried out for different concentrations of HMF solutions (from 2 to $15 \mathrm{mg} / \mathrm{L}$ ). The fitted calibration curve is: $A_{284 n m}=0.1174 \cdot c_{H M F}[\mathrm{mg} / \mathrm{L}]$. The product molar yield was evaluated according to the equation: yield $=[h] /[f]_{0} \cdot 100$, where $[f]_{(0)}$ is the initial fructose concentration and $[h]$ is the HMF final concentration. 


\subsection{Results and discussion}

The sputtered zirconia layer has a dense structured as visualized by HRSEM (Figure 4.5). The 1:2 elemental stoichiometry for $\mathrm{Zr}$ :O is constant for a wide range of $\mathrm{O}_{2}$ concentrations used during the sputtering process. The elemental content was measured with both XPS and EDX. The observed drop in deposition rate for high $\mathrm{O}_{2}$ concentrations is attributed to the oxidation of the target prior to sputtering (Figure 4.6). For the final recipe, a 7.5\% (vol.) $\mathrm{O}_{2}$ was chosen to ensure a high deposition rate which results in a $250 \mathrm{~nm}$ layer for a 40 min deposition time as shown in Figure $4.5 \mathrm{a}$. and b. XRD shows a mixture of tetragonal and monoclinic crystalline phases. The annealing process increases the crystallinity of the film, with the previous amorphous phase transitioning to the tetragonal structure which shows up in an increase in the $\mathrm{T}(1,1,1)$ peak intensity. TEM revealed a polycrystalline film (Figure 4.5), as sputtering is a high energy process which does not give the possibility for preferential orientation. The wash coated titania film displays a high degree of homogeneity (Figure 4.7). While the initial suspension shows already a narrow size distribution, $157 \pm 70 \mathrm{~nm}$, there are small aggregates comprising of monodisperse particles of $21 \mathrm{~nm}$ which are the building blocks of the final coating as it is shown in Figure 4.7. A porosity of $45 \%$ and roughness similar to the particle radius $(\sim 8 \mathrm{~nm})$ was measured by Spectroscopic Ellipsometry. The specific surface area of the porous film given the particle size and the density of anatase $\left(3895 \mathrm{~kg} / \mathrm{m}^{3}\right)$ is about $50 \mathrm{~m}^{2} / \mathrm{g}$. The crystalline phase is not affected by the sintering process. The $80 \%$ anatase to $20 \%$ rutile composition specified by the manufacturer is preserved. The high quality of the commercial suspension and the constant displacement rate ensured a uniform thickness of the wash coated catalyst which can be observed in the cross section of the channel (Figure 4.7 c). A displacement rate of $17 \mathrm{~mm} / \mathrm{sec}$ led to a thickness of $5 \pm 0.5 \mu \mathrm{m}$.

The reaction was performed at 7 bar and $130{ }^{\circ} \mathrm{C}$ for different flowrates. The product (HMF) absorption peak was monitored at each corresponding residence time (Figure 4.8). Given the small product yield (Figure 4.9), the conversion is reaction rate limited and external mass transfer does not have to be taken into account. This assumption is valid for the second Damköhler number, $D a_{I I}<10^{-1}$, which varies for the fitted reaction constants between $4 \times 10^{-3}$ and 0.013 . 

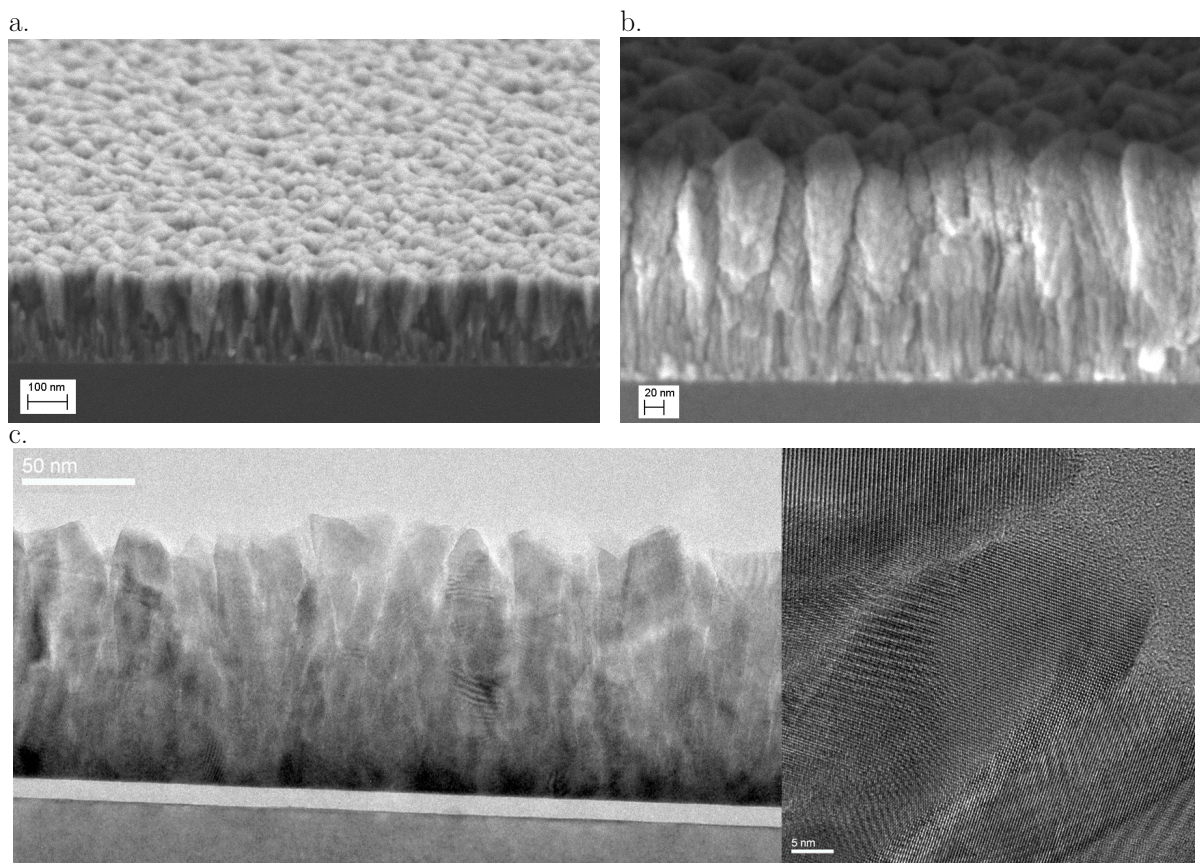

Figure 4.5: a. High resolution SEM of a $250 \mathrm{~nm} \mathrm{ZrO}_{2}$ layer b. Higher SEM magnification revealing the dense structure c. TEM of sputtered $\mathrm{ZrO}_{2}$ displaying its polycrystalline morphology

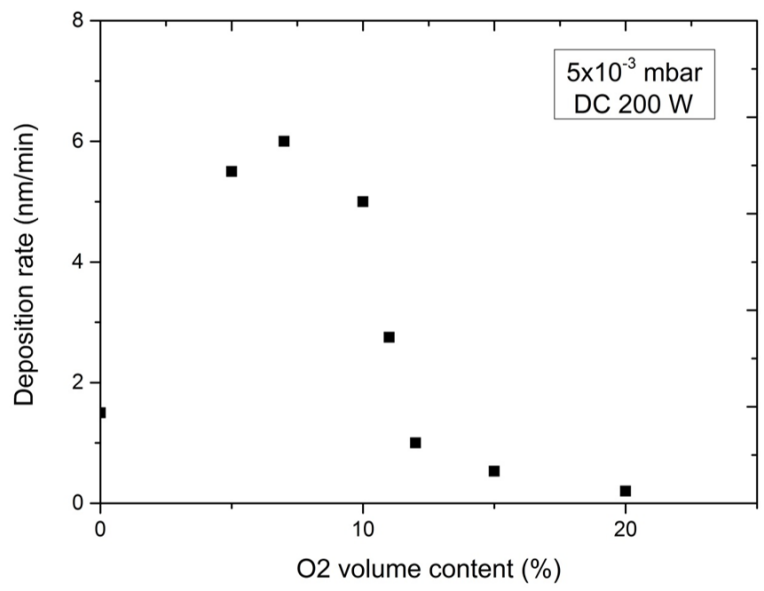

Figure 4.6: Deposition rate dependency on volumetric gas composition 

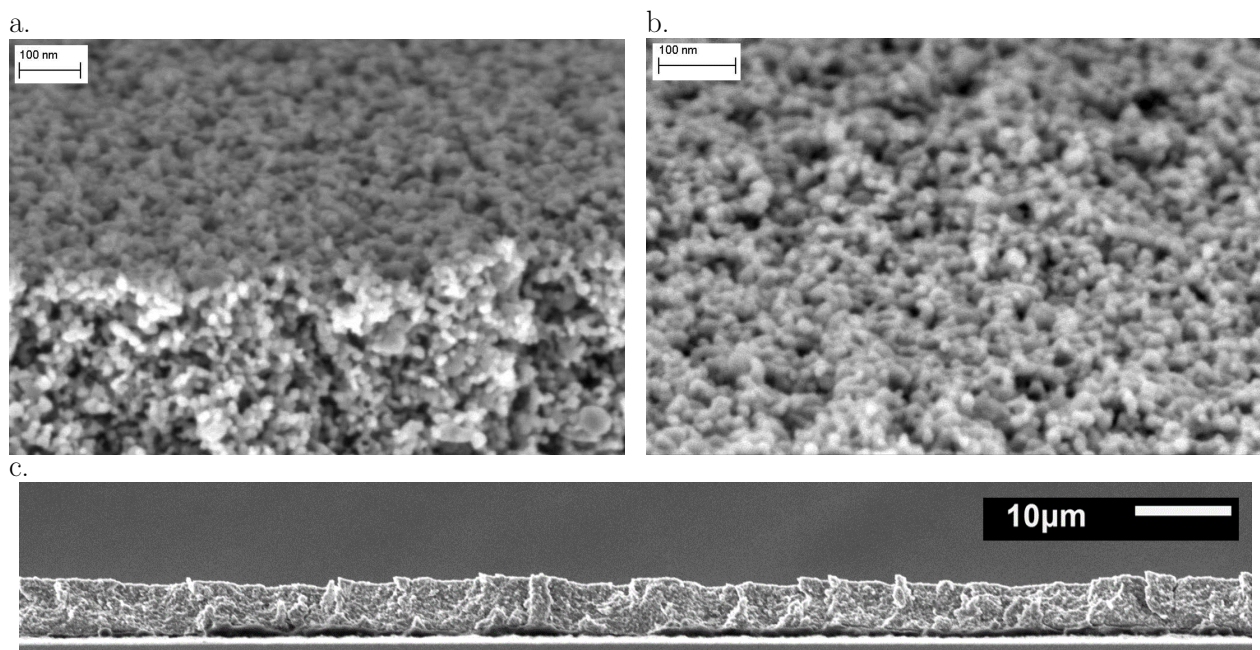

Figure 4.7: High resolution $\mathrm{SEM}$ of $\mathrm{TiO}_{2}$ showing a. the porous structure and b. the monodisperse particles c. Cross-section of the wash coated uniform layer

$$
D a_{I I}=\sqrt{\frac{k}{D^{0}}} \cdot L
$$

where $k$ is the volumetric reaction rate constant $[1 / \mathrm{s}]$ for a first order reaction rate, $L$ is the height of the channel, and $D^{0}$ is the diffusion coefficient of fructose in water corrected for the change in temperature and pressure by using the Stokes Einstein equation:

$$
D^{0}=\frac{k_{B} T}{6 \pi \eta^{0} a}
$$

$k_{B}$ and $\eta^{0}$ are Boltzmann constant and the viscosity of pure water at temperature $T$ and the corresponding pressure ( 7 bar). $a=0.365 \mathrm{~nm}$ is the effective hydrodynamic radius of fructose in water at small concentrations reported in Lee et al. [169]. The variation in $a$ with temperature is small, typically less than $3 \%$. Consequently, a homogeneous concentration profile can be assumed in the transversal direction which simplifies the system to a plug flow reactor (PFR) model. A first order reaction rate assumption was used due to the low concentration range. 


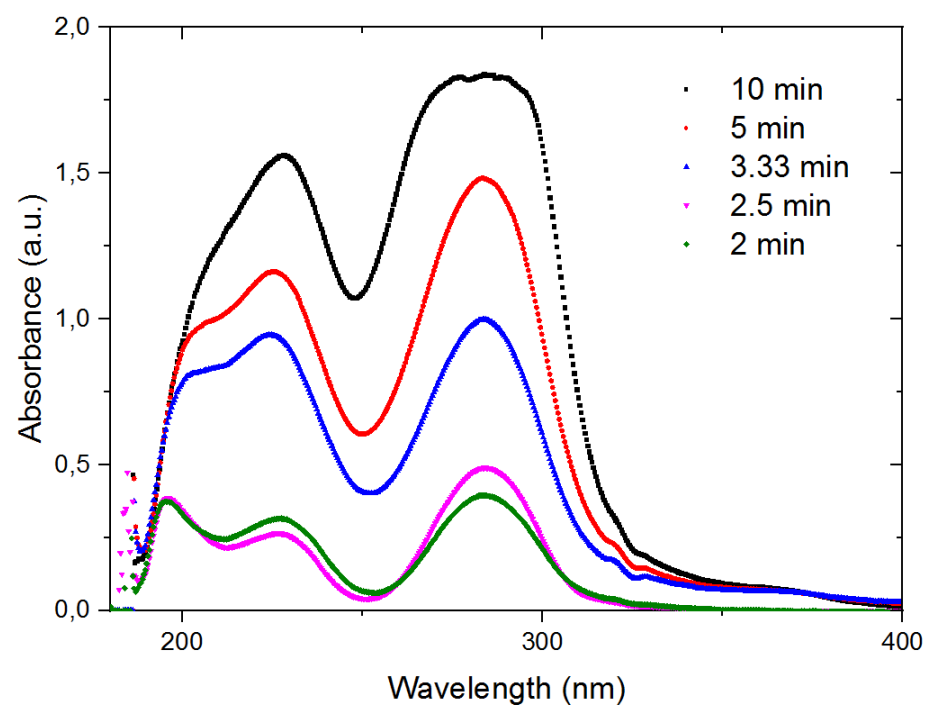

Figure 4.8: HMF absorbance showing the increase in HMF production for higher residence times. Reaction performed at $130{ }^{\circ} \mathrm{C}$ and 7 bar using the porous $\mathrm{TiO}_{2}$ layer

$$
\begin{gathered}
\frac{d[f]}{d t}=-k[f] \\
\ln \frac{[f]}{[f]_{0}}=\ln \frac{[f]_{0}-[h]}{[f]_{0}}=-k t \\
\frac{[h]}{[f]_{0}}=1-e^{-k t}
\end{gathered}
$$

We take into account only the reaction pathway to HMF, as the present spectroscopic measurement allows for HMF detection only. Mass spectrometry is required to identify other possible secondary products and quantify the absolute conversion of fructose.

The sputtered $\mathrm{ZrO}_{2}$ displayed a low activity $\left(k=1.43 \times 10^{-3} 1 / \mathrm{min}\right)$ which referenced to the geometric surface area of the film converts to a surface reaction rate constant of $k^{\prime \prime}=1.192 \times 10^{-9} \mathrm{~m} / \mathrm{s}$. The slow overall kinetics can be 

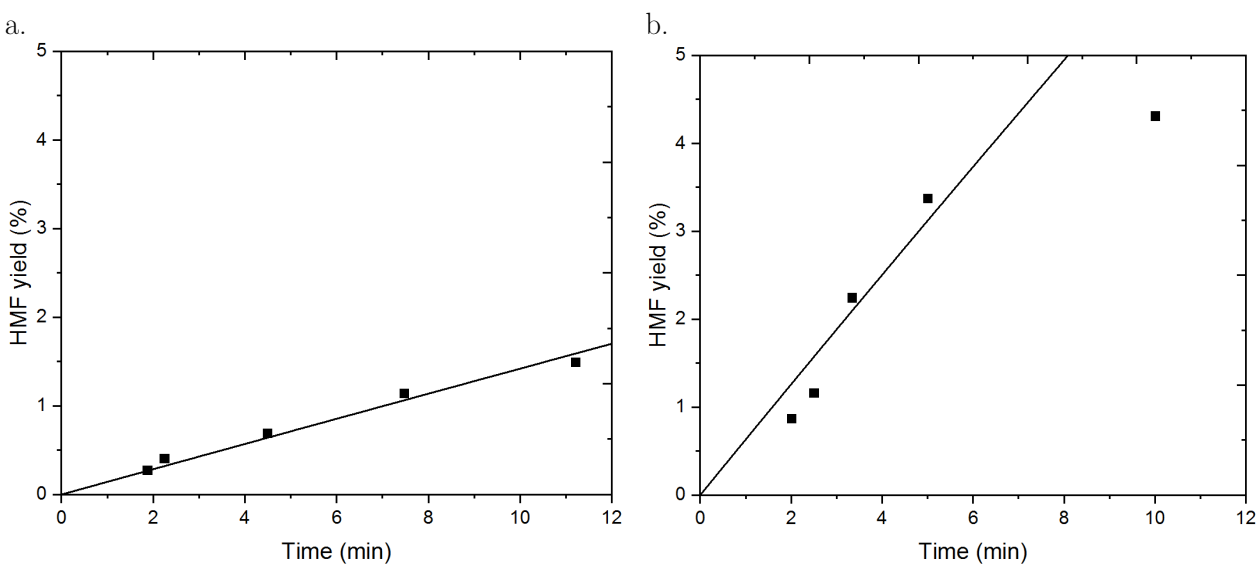

Figure 4.9: HMF yield dependence on residence time for a. dense $\mathrm{ZrO}_{2}$ and b. porous $\mathrm{TiO}_{2}$. The data points represent the experimental measurements. The continuous line represents equation 5 where a. $k=1.43 \times 10^{-3} 1 / \mathrm{min}$ and $\mathrm{b}$. $k=6.34 \times 10^{-3} 1 / \mathrm{min}$

attributed to the obvious low surface available area, but also to a low density of active sites.

The next attempt tried to improve both aspects, increase the specific surface area and alter the surface functionality for an increase in the number of acid sites. The initial concentration of fructose was lowered to $0.5 \mathrm{~g} / \mathrm{L}$ in the case of $\mathrm{TiO}_{2}$ due to the absorption signal saturation beyond HMF concentrations of $15 \mathrm{mg} / \mathrm{L}$. Figure 4.8 shows the full spectrum of the steady state for each residence time. The HMF absorption corresponding to the conversion for $10 \mathrm{~s}$ residence time has a much broader peak which proves that it does not reflect the true corresponding concentration. Hence, the data for this measurement was not used in the kinetic fitting.

The porous titania layer displayed a higher volumetric rate constant due to the higher available surface area, $k=6.34 \times 10^{-3} 1 / \mathrm{min}$. The extracted value is averaged with respect to the height of the channel. In order to be representative for the catalyst layer, it needs to be normalized to the thickness of the layer with respect to the height of the channel, $k_{\mathrm{TiO}_{2}}=k \cdot L / \delta$, where $\delta$ is the catalyst thickness. To be able to validate the volume averaging of the reaction rate constant, we need to exclude internal mass transfer limitations, such that the catalyst layer is utilized evenly throughout its thickness. Two parameters can be used in this respect: thiele modulus, $\phi$, and the internal 
effectiveness factor, $\eta$. Thiele modulus evaluates the reaction time scale with respect to the diffusion time scale:

$$
\phi=\sqrt{\frac{k_{\mathrm{TiO}_{2}}}{D_{\text {eff }}}} \cdot \delta
$$

Where $D_{\text {eff }}=D^{0} \cdot \frac{\epsilon}{\tau}$ is the effective diffusion coefficient, $\epsilon=0.45$ is the porosity and $\tau=1.35$ is the tortuosity [67]. $\phi$ values up to $6 \times 10^{-3}$ for the extracted kinetics confirmed the reaction driven regime for which the formal criterion is $\phi<10^{-1}$. The effective factor gives the ratio between the net reaction rate and the surface reaction rate, namely the rate in the absence of concentration gradients, which for the present system is almost unity:

$$
\eta=\frac{\tanh \phi}{\phi}
$$

The conversion from $k_{\mathrm{TiO}_{2}}$ per unit volume $\left[\mathrm{s}^{-1}\right]$ to $k^{\prime \prime}$ per units surface area $[\mathrm{m} / \mathrm{s}]$ has the known formula: $k^{\prime \prime}=k_{\mathrm{TiO}_{2}} /\left(S_{a} \rho(1-\epsilon)\right)$, where $S_{a}=5 \times 10^{4}$ $\mathrm{m}^{2} / \mathrm{kg}$ is the specific surface area, $\rho=3895 \mathrm{~kg} / \mathrm{m}^{3}$ is the anatase density and $\epsilon=0.45$ is the porosity. This gave a lower surface reaction rate constant, $k=9.87 \times 10^{-12} \mathrm{~m} / \mathrm{s}$, than the corresponding value for the $\mathrm{ZrO}_{2}$ layer, proving a lower density of the $-\mathrm{OH}$ functional groups required for the reaction mechanism.

The dependency of $k$ on temperature is expressed by the Arrhenius equation:

$$
k=A e^{-E_{a} / R T}
$$

where $E_{a}$ is the activation energy, $A$ is the pre-exponential factor and $R=$ $8.31446 \mathrm{Jmol}^{-1} \mathrm{~K}^{-1}$ is the ideal gas constant. We investigated experimentally this dependency of $k$ on temperature for the porous $\mathrm{TiO}_{2}$ layer for which we determined $E_{a}=80 \mathrm{~kJ} / \mathrm{mol}$ and $A=e^{19.4} 1 / \mathrm{min}$ (Figure 4.10). These values are slightly higher than what Carnity et al. measured for the same reaction using a niobium phosphate catalyst $\left(E_{a}=65.8 \mathrm{~kJ} / \mathrm{mol}\right.$ and $A=e^{15.7}$ $1 / \mathrm{min}$ ) [183]. For these measurements, the surface functionalization procedure using $\mathrm{H}_{3} \mathrm{PO}_{4}$ acid under UV exposure was extended from 4 to $6 \mathrm{~h}$. This change led to a modest improvement in the reaction rate constant, namely from $6.34 \times 10^{-3} 1 / \mathrm{min}$ to $11 \times 10^{-3} 1 / \mathrm{min}$ for $130^{\circ} \mathrm{C}$. We did not proceed to quantify the surface functionality of $\mathrm{TiO}_{2}$. 


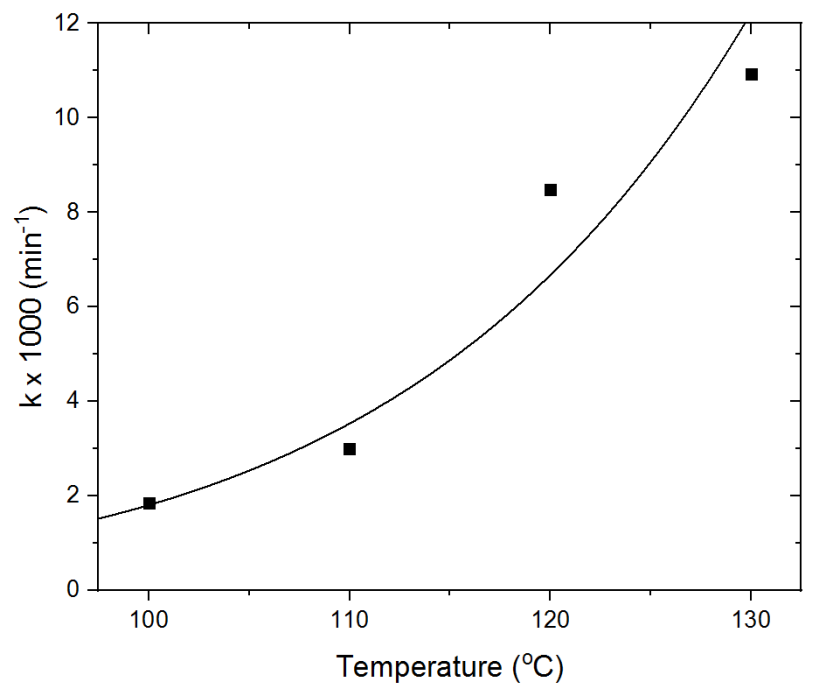

Figure 4.10: The dependency of $k$ with temperature. The data points represent the experimental measurements. The continuous line represents equation 6 where $E_{a}=80 \mathrm{~kJ} / \mathrm{mol}$ and $A=e^{19.4} 1 / \mathrm{min}$

While the catalysts investigated in this work displayed rather low activities, the microfluidic platform proved to be a useful tool for rapid investigation of catalytic performance. Especially for higher activities when mass transport becomes limiting and accurate modelling is crucial to decouple the kinetics from reactor design, microreactors provide a reliable option due to their well defined fluid dynamics.

\subsection{Conclusion}

A microfluidic platform was developed for high temperature, high pressure conversion with an inline UV-Vis spectroscopic measurement that facilitates the fast screening of catalytic materials. The well-defined mass transport characteristic for immobilized catalytic layers in microchannels allows for accurate kinetic investigation. The dehydration of fructose to 5-hydroxymethyl2-furaldehyde (HMF) was studied using both sputtered $\mathrm{ZrO}_{2}$ and wash coated $\mathrm{TiO}_{2}$ layers. The kinetics were determined for each catalyst. For the $\mathrm{TiO}_{2}$ layer, that showed higher conversion, the dependency on temperature was also 
investigated, revealing an activation energy of $80 \mathrm{~kJ} / \mathrm{mol}$. Surface functionalization of $\mathrm{TiO}_{2}$ using phosphoric acid treatment under UV light proved to have a limited capacity for increasing the density of active sites. 



\section{5 \\ Reaction driven diffusio-osmotic flow inside catalytic dead-end pores ${ }^{\circ}$}

We study convective transport inside a catalytic dead-end pore resulting from reaction driven diffusio-osmotic flows. This additional mass transport mechanism does not require any external input, but arises spontaneously when the configuration of the catalyst matrix facilitates the development of significant gradients with respect to the bulk solution. The surface driven flow originates from osmotic pressure gradients and diffusion potential in case of charged species. We have performed experimental studies on reaction driven flows inside a microfluidic dead-end channel with a photocatalytic wall. This diffusio-osmotic flow is a result of the generated concentration gradient of glycerol and its degradation products formed by the photocatalytic reaction. This catalytically generated flow was characterised by 3D particle tracking and compared to a numerical mass and momentum model including diffusio-osmotic slip.

${ }^{\circ}$ Submitted as: Aura Visan, Alvaro Marin, Jeffery A. Wood and Rob G.H. Lammertink, Reaction driven diffusio-osmotic flow inside catalytic dead-end pores (2019). 


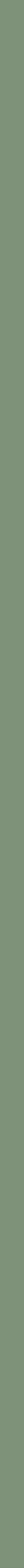




\subsection{Introduction}

Mass transport in dead-end pores is of great importance in many chemical systems, for example heterogeneous catalysis, enhanced oil recovery, and wet etching processes, amongst others. For incompressible liquids, advective transport into dead-end pores is challenging, as pressure driven flow is not possible. Regarding enhanced oil recovery, this results in oil residues remaining inside dead-end channels in porous structures. In heterogeneous catalysis, the lack of advection inside dead-end pores causes internal concentration polarization, i.e. depletion of reactant deeper inside the catalyst particle. The transport of reactive species into, and products out of, the catalytic particle is then frequently limiting the efficiency of the catalyst.

The main strategy to reduce internal concentration polarization has been to decrease the diffusion length scale [184]. The only available design option here is to dimension the particle/agglomerate size [185] or layer thickness [67] according to the chemistry at hand. The kinetics will dictate how much of the catalyst is being actually utilised, such that the only intervention is to minimise material overuse. Well-known parameters such as Thiele modulus and the internal effectiveness factor evaluate this length scale [90,91,95].

Recently, chemical gradients were demonstrated for obtaining advective flows via "transient diffusio-osmosis" in dead-end channels. [26, 27] The transient nature is caused by the flow itself which mixes the solution and therefore diminishes any chemical gradient. The concept of diffusio-osmosis was first introduced by Derjaguin et al. $[2,3]$ who demonstrated that a solute gradient along a surface will develop osmotic pressure gradients within the interfacial layer where the interaction potential spans, which will set the fluid in motion. Another contribution to this driving force in case of charged species is the diffusion potential, namely the electric potential that develops for ion concentration gradients based on the different diffusivities of the corresponding ions [4]. There is a large body of work that extended this theory further $[5-9,186]$ and validated it with increasingly more accurate experiments $[10-13,15,18,19,187,188]$.

The strong understanding of this phenomenon led to the proposal of a range of applications from surface charge characterisation [189], particle separation based on their zeta potential [15-17, 28,29], membrane fouling prevention [23-25], enhanced oil extraction [26], energy production from salinity gradients [30] and fluid control in micro-channels [32]. Most of these examples are 


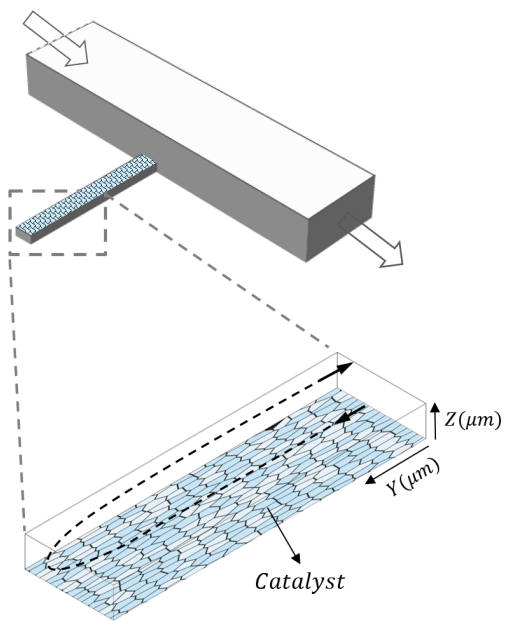

b.

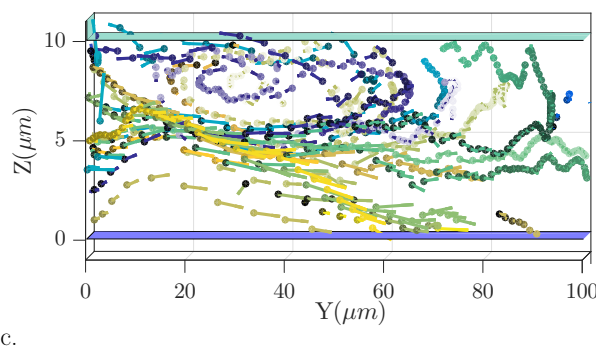

c.

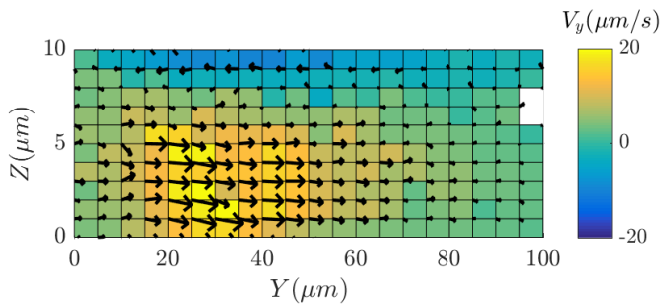

Figure 5.1: a. Sketch of the setup b. Particle trajectories c. Particle velocities in the $y z$ plane

based on externally applied gradients by bringing in contact solutions of different concentration. The second approach is in-situ gradient generation by sacrificial substrates that are either consumed, for example, by depolymerization reactions [41] or depleted such is the case of ion-exchange resins [190]. Another example is the use of redox chemistry on bimetallic surfaces [34,37,191-194]. The production and consumption of protons on adjacent noble metals leads to an uneven distribution of protons and hence an external electric field which sets in motion electrophoretically the particles. The mechanism relies on particular chemicals, namely hydrogen peroxide or hydrazine, used as fuel to provide the corresponding transport.

Our work focuses on studying induced advective flows in catalytic porous structures with the aim of enhancing mass transport in such systems. It proves the inherent existence of diffusio-osmosis in reactive dead-end pores where conditions for this flow arise spontaneously. The catalytic reaction will generate the required chemical gradient by itself, as well as potentially sustain it. We have analysed the catalytic induced flow by means of 3D particle tracking inside a microchannel comprising a reactive wall. A numerical model is proposed to understand the signature of this flow in terms of the generated gradient and solute-wall interactions. 


\subsection{Experimental}

A glass wafer was patterned using standard photolithography without HMDS pretreatment. Following development and UV ozone cleaning, the wafer was dried for $15 \mathrm{~min}$ at $120{ }^{\circ} \mathrm{C}$. To prevent rehydration, the wafer was hydophobized immediately with FDTS (Sigma Aldrich) in vapour phase using a vacuum chamber. The best results were achieved when the wafer was left for a few days to allow for covalent bonding completion. A thermal treatment at $60{ }^{\circ} \mathrm{C}$ removed the excess FDTS from the surface. The final water equilibrium contact angle for the hydrophobic regions was $115 \pm 5^{\circ}$. Prior to dip coating, the wafer was dipped for a minute in $99 \%$ nitric acid to remove the photoresist and ensure a clean hydrophilic surface. This short treatment had a negligible influence on the hydrophobic functionality with a decrease in the water equilibrium contact angle up to $5^{\circ}$. Dip coating was performed using a commercial $30 \mathrm{wt} \% \mathrm{TiO}_{2}$ (Evonik) suspension at a speed of $10 \mathrm{~mm} / \mathrm{min}$ which gave for $600 \mu \mathrm{m}$ wide stripes a thickness of $1100 \mathrm{~nm}$ determined using a surface profiler. The patterned glass wafer was sintered to improve adhesion for $2 \mathrm{~h}$ at $500{ }^{\circ} \mathrm{C}$ in air. The heating and cooling rates were kept at $2{ }^{\circ} \mathrm{C} / \mathrm{min}$. The microreactor was assembled by aligning the $\mathrm{TiO}_{2}$ patterns to the side dead-end channels replicated in a PDMS slab from a microstructured mold. The PDMS was prepared by blending the polymer base (RTV-615 A, Permacol) with the curing agent (RTV-615 B, Permacol) in 10:1.5 ratio to increase stiffness. The mixture was poured on top of a patterned wafer, degassed and cured for $2 \mathrm{~h}$ at $80{ }^{\circ} \mathrm{C}$. The PDMS was kept in DI water prior to bonding to prevent water permeation during the experiments. After cleaning for $30 \mathrm{~min}$ in $2 \mathrm{M} \mathrm{NaOH}$ solution, the permanent bonding between the $\mathrm{TiO}_{2}$ patterned substrate and the PDMS was achieved by activating the PDMS and glass surface using $\mathrm{O}_{2}$ plasma for 12 seconds at $100 \mathrm{~W}$. The design was inspired by the work of Shin et al. [189]. The device has a large main channel with height of $100 \mu \mathrm{m}$ and $600 \mu \mathrm{m}$ width and side dead-end pores with height of $10 \mu \mathrm{m}, 500$ $\mu \mathrm{m}$ width and $300 \mu \mathrm{m}$ length.

A 0.01 wt\% glycerol (Sigma Aldrich) solution seeded with $2.5 \times 10^{-3} \mathrm{wt} \%$ tracer particles (PS-FluoRot-Fi267 $1 \mu \mathrm{m}$ Microparticles GMBH) was flushed through the main channel using a syringe pump. The side dead-end channels filled by capillary forces due to the hydrophilic walls, while air could could be evacuated through the PDMS matrix. The reaction was triggered by UV light of different intensities $\left(18-90 \mathrm{~mW} / \mathrm{cm}^{2}\right)$. Density driven flows were ruled out 
by performing a blank experiment using the same catalytic configuration with only DI water under UV light illumination. Wide channels were chosen to increase the number of data points. However, this introduced disturbances in the side capillaries caused by the flow in the main channel and therefore during measurements the flow was stopped in the main channel which introduces a transient in the inlet boundary condition. To exclude this effect only the first 20 second of the experiment were used for processing. The wide side channels allowed flushing them with fresh solution between experiments. The homogeneous concentration profile was verified by the absence of directional particle movement when the pumping was stopped. The zeta potential of the tracer particles $(-45 \pm 5 \mathrm{mV})$ was measured in the $0.01 \mathrm{wt} \%$ glycerol solution using a Malvern Zetasizer NanoZS .

The particle tracking was performed with a commercial LaVision system that uses a dual-cavity Nd:YAG laser at $532 \mathrm{~nm}$. Images were recorded at 10 frames per second using a double-shutter PCO Sensicam camera with a resolution of $1,376 \times 1,040$ pixels $\times 12$ bits. Bright-field images were acquired to determine the locations of the catalyst patch with respect to the entrance of the deadend side channel. The particle velocity was resolved in 3D using the General Defocusing Particle Tracking (GDPT) technique [195]. This method is based on a set of calibration images and the normalized cross-correlation function used to connect the 2D intensity map with the height position.

\subsection{Model}

Momentum and mass transport are solved numerically for a two-dimensional domain of height $H$ and length $L$ consisting of a solution of glycerol which is consumed at the bottom catalytic surface and connected to the large reservoir at the left side. The large reservoir was also modelled at the left side of the catalytic pore to avoid fictitiously large reactant and, especially, product concentration gradients. The height is $10 H$, while the length is $L / 3$.

The system is governed by the Stokes and the continuity equations for fluid dynamics. The inertial term is neglected due to small Re numbers. The species conservation equation for mass transport includes advection and diffusion. In dimensionless form they are given by: 


$$
\begin{gathered}
\frac{1}{S c} \frac{\partial \boldsymbol{u}}{\partial t}=-\nabla p+\nabla^{2} \boldsymbol{u} \\
\nabla \cdot \boldsymbol{u}=0 \\
\frac{\partial c_{i}}{\partial t}=\nabla \cdot\left(D_{i} \nabla c_{i}-\boldsymbol{u} c_{i}\right)
\end{gathered}
$$

The Schmidt number is $S c=\nu / D_{1}$ where $\nu$ is the kinematic viscosity, $t=\widetilde{t} / t_{0}$ where $t_{0}=H^{2} / D_{1}$ is the diffusion time with $D_{1}=9.3 \times 10^{-10} \mathrm{~m}^{2} / \mathrm{s}$ the diffusivity of glycerol [196] and $p=\widetilde{p} /\left(\nu \rho D_{0} / H^{2}\right)$ is the pressure. The dimensionless velocity $\boldsymbol{u}$ is obtained with respect to the diffusion velocity $\boldsymbol{u}=\widetilde{\boldsymbol{u}} / D_{0} H$, where the height of the dead-end channel is $H=10 \times 10^{-6} \mathrm{~m}$ and the length $L=300 \times 10^{-6} \mathrm{~m} . c_{i}=\widetilde{c}_{i} / c_{0}$ is the dimensionless concentration where $i=1$ refers to the reactant species and $i=2$ represents the product and $c_{0}$ the initial glycerol concentration. The diffusivities are referenced with respect to the reactant diffusivity, $D_{i}=\widetilde{D_{i}} / D_{1}$, where $D_{2}=1.94 \times 10^{-9} \mathrm{~m}^{2} / \mathrm{s}$ is the diffusivity of carbon dioxide. Both catalytic surface and top wall are defined by the diffusio-osmotic slip caused by the reactant and product concentration gradients. The phoresis of the particles follows the same equation while changing sign as they are propelled in the opposite direction by the flow enclosing the particle. We want to stress here, that this corresponds to cases where the pore radius is large compared to the solute interaction length.

$$
u_{j}^{D O F}=-\frac{\mu_{j}^{R}}{c_{1}} \frac{\partial c_{1}}{\partial x}-\frac{\mu_{j}^{P}}{c_{2}} \frac{\partial c_{2}}{\partial x}
$$

The mobility $\mu$ is the prefactor of the diffusio-osmotic slip which lumps the effects of the diffusion potential and interaction strength between reactant molecules and the catalyst surface (quantified via the zeta potential in case of charged species or a more general integrated interfacial concentration $[2,5]$ ). We reference the mobility to the diffusivity of the reactant, $\mu=\widetilde{\mu} / D_{1}$. Indices $R$ and $P$ stand for reactant and product species. Indices $j$ corresponds to the 3 type of surfaces present in this system, $\mathrm{TiO}_{2}$ (bottom surface), PDMS (top surface) and PS particles. 
(a)

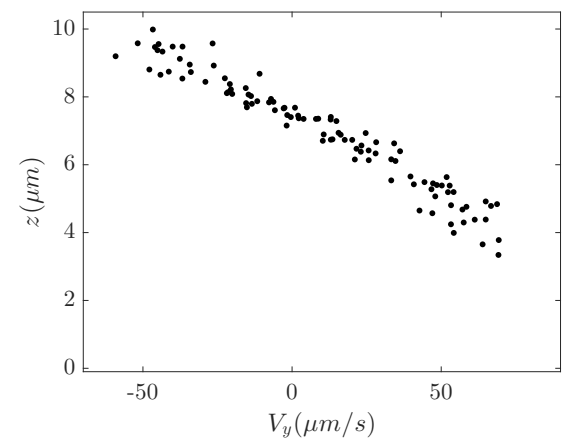

(c)

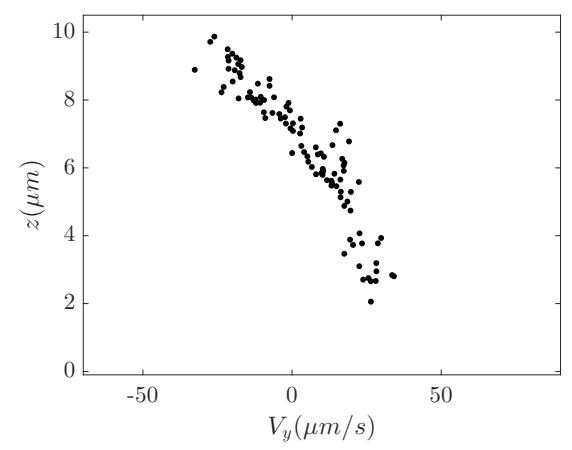

(b)

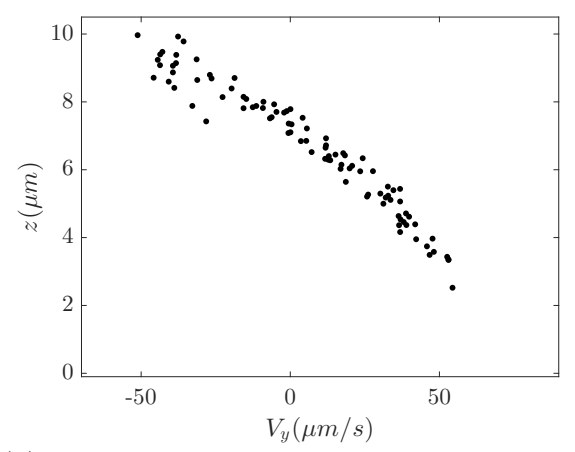

(d)

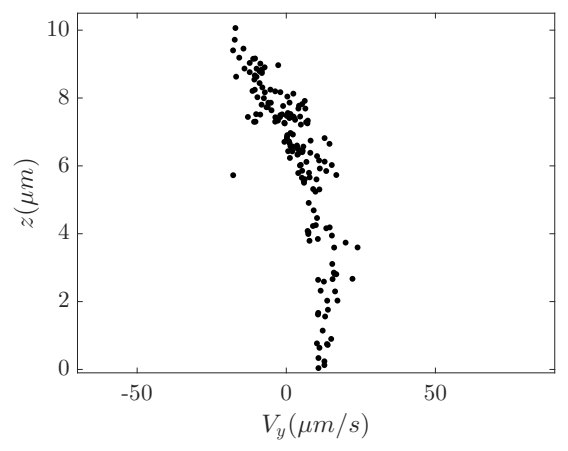

Figure 5.2: Velocity profile across the capillary integrated for the first $10 \mathrm{~s}$ at (a) $20 \leq y \leq 30 \mu m$, (b) $40 \leq y \leq 50 \mu m$, (c) $60 \leq y \leq 70 \mu m$, (d) $80 \leq y \leq 90 \mu \mathrm{m}$ 
(a)

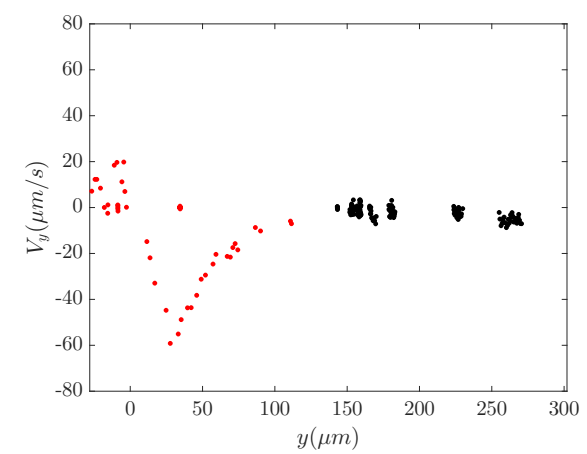

(c)

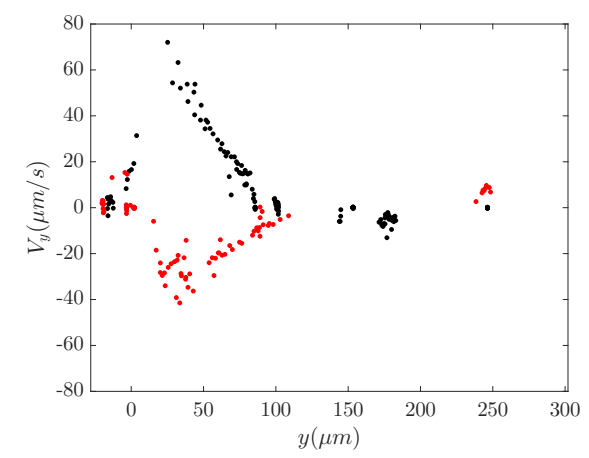

(b)

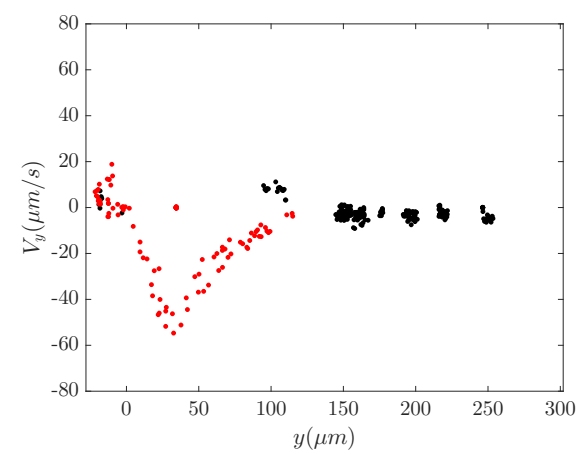

(d)

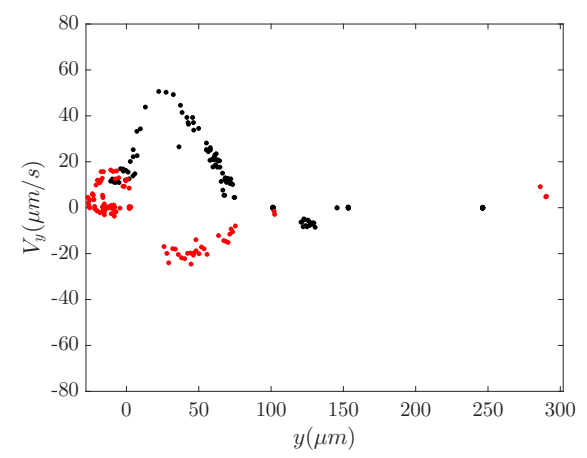

Figure 5.3: Surface velocity along the catalyst (black markers) integrated over $0 \leq z \leq 2 \mu \mathrm{m}$ and along the PDMS wall (red markers) integrated over $9 \leq z \leq 10 \mu m$ for (a) $1.5 \leq t \leq 4.5 \mathrm{~s}$, (b) $5 \leq t \leq 8 \mathrm{~s}$, (c) $10.5 \leq t \leq 13.5 \mathrm{~s}$, (d) $17.5 \leq t \leq 20.5 \mathrm{~s}$

For the species transport, the inlet boundary condition at the left side of the large reservoir is defined by a fixed bulk concentration, $c_{1}=1$ and $c_{2}=0$. The boundary condition for catalyst surface is represented by the normalised surface flux which is a first order reaction rate, $\frac{\partial c_{1}}{\partial y}=-\frac{\partial c_{2}}{\partial y}=-D a_{I I} c_{1}$, where $D a_{I I}=k H / D_{1}$ is the dimensionless second Damköhler number with surface reaction rate constant $k$, while the inactive regions, top and right side inside the capillary and the rest of walls of the large reservoir, have a no flux boundary condition, $\frac{\partial c_{i}}{\partial y}=0$.

It has been reported in literature [197] that the photocatalytic oxidation of glycerol leads to 4 main products with the following molar distributions: $8 \%$ glyceraldehyde, $6 \%$ 1,3 - dihydroxyacetone, $22 \%$ formic acid and $64 \% \mathrm{CO}_{2}$. 
This distribution changes with the residence time and complete kinetic data is not available. The individual interaction potentials of these species with the catalytic surface are also unknown. Thus, we cannot determine their individual contribution to the net diffusio-osmotic driving force. This will be part of future work, as an online mass spectrometer is required to investigate the reaction mechanism and the kinetics of the elementary reactions. To account for observations, the individual product species were collapsed into a net contribution which regards the predominance of one of them, namely $\mathrm{CO}_{2}$ whose diffusivity was used as the product in the model.

The governing equations are solved using Finite Element Analysis in COMSOL Multiphysics 5.3. The simulations were performed with an unstructured mesh with triangular elements for the large reservoir domain and a structured mesh for the capillary domain. 150 by 100 rectangular elements were used within the capillary to ensure mesh independence. The physical properties of the medium were taken as water at $298 \mathrm{~K}$ due to the dilute nature of the solution.

\subsection{Results and discussion}

Initially, the dead-end channel has the same concentration as the main channel which is confirmed by the lack of directional motion of the tracer particles. Once the reaction is triggered at the surface of the catalyst by UV illumination, glycerol degradation is initiated which lowers its concentration within the capillary. This reactant concentration gradient together with the product counterpart develops along the capillary and generates flow at both the catalyst surface and the upper wall. Although the PDMS wall is inactive, the diffusion time scale across the capillary is small compared to the kinetic analogue, providing a similar concentration gradient along the top boundary. The resulting fluid recirculation is visualised by tracer particles. The flow field has been resolved in 3D using General Defocusing Particle Tracking. An experiment with the same configuration of the catalyst and only DI water displayed no directional motion under UV illumination. This rules out density driven flows due to local heating and any additional dynamics driven by gradients generated by solvent (water) reactions. Also, the $1 \mathrm{mM}$ glycerol concentration ensures negligible change in density and other physical properties upon chemical conversion.

The particle velocities across the height at different position along the capillary are shown in Figure 5.2. These tracer particles sample the flow, but also have 
a.

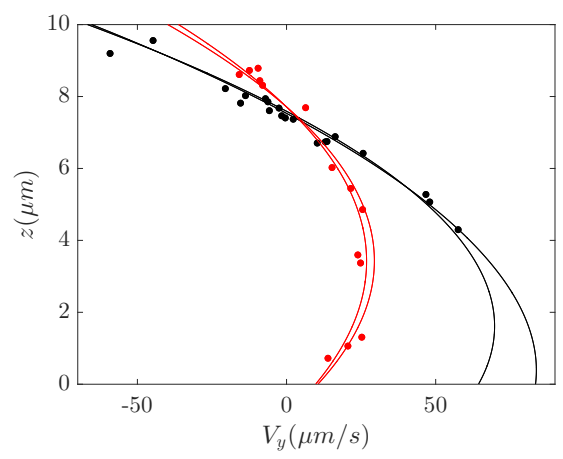

b.

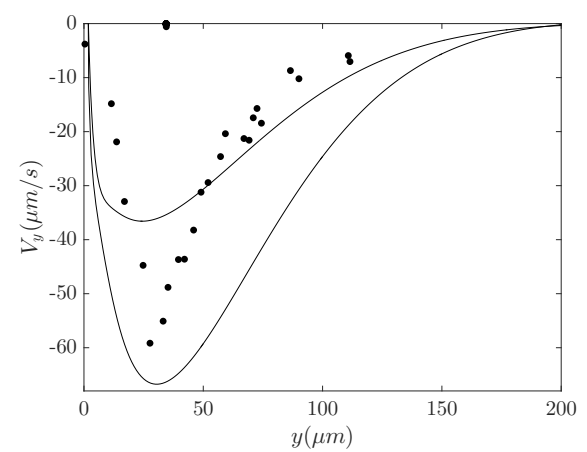

Figure 5.4: a. Velocity profile inside the pore for the first $4.5 \mathrm{~s}$ at $24 \leq y \leq 29$ $\mu m$ (black markers) and at $77 \leq y \leq 82 \mu m$ (red markers). The continuous lines depict the simulation results at the corresponding positions to confirm the integration range b. Surface velocity along the PDMS integrated over $9 \leq y \leq 10 \mu \mathrm{m}$ (black markers) and the simulation results for $z=9 \mu \mathrm{m}$ and $z=10 \mu \mathrm{m}$ at $\mathrm{t}=4.5 \mathrm{~s}$.

their own diffusio-phoretic motion due to the same diffusio-osmotic flow at the surface of the particles which propels them in the opposite direction. The magnitude and the direction of these surface flows will depend on the kinetics of the reaction and the interaction potential between the solute species and the solid surface. There is an inward flow at the catalyst surface and an outward flow at the upper wall. This reversed flow direction comes from a different interaction between the solute species and the catalyst versus the PDMS surface. The difference in interaction potential originates from the different surface charge between substrates. At the working $\mathrm{pH}(1 \mathrm{mM}$ glycerol solution in DI water has a $\mathrm{pH}=5.5)$, the surface of $\mathrm{TiO}_{2}$ is expected to be positive $\left(p K_{a}=7.5[198]\right)$, while PDMS $\left(p K_{a}=5\right.$ [199]) and PS tracer particles have a negative surface charge. However, the surface charge is also affected by the cleaning procedure and in the case of PDMS by the $\mathrm{O}_{2}$ plasma treatment required for bonding [200].

The velocities close to surface of the catalyst and PDMS along the capillary are shown in Figure 5.3 at different time intervals. Close to the entrance of the capillary the solute concentration gradient vanishes due to the large reservoir. The surface induced velocity peaks around $\mathrm{y}=30 \mu \mathrm{m}$ followed by a further decrease. This particular surface velocity profile is dictated by the interplay between the driving force generated by the reactant gradient and the 


$$
\begin{array}{|c|c|c|c|c|c|c}
k[\mathrm{~m} / \mathrm{s}] & \mu_{\mathrm{TiO}_{2}}^{R} & \mu_{\mathrm{TiO}_{2}}^{P} & \mu_{\mathrm{PDMS}}^{R} & \mu_{\mathrm{PDMS}}^{P} & \mu_{\mathrm{PS}}^{R} & \mu_{\mathrm{PS}}^{P} \mid \\
2 \times 10^{-6} & -12.2 & -8.7 & -16.4 & -1.6 & -1.2 \mid 1.5
\end{array}
$$

Table 5.1: Fitted reaction rate constant and mobilities

opposing product gradient which becomes more significant as the conversion proceeds. This dependency of the velocity on the conversion degree produces a velocity variation along the pore length (Figure 5.2). The same dependency on conversion also causes a sharp transient (Figure 5.3) in which the velocity decreases significantly and even changes direction at the catalyst surface as the product driving force overcomes the reactant contribution.

We have analysed the velocity field using a numerical model (for details, see the methods section). The model is based on the mass transport of reactant and product species by advection, diffusion and reaction. The fluid dynamics are driven by the surface flows at the top and bottom walls which arise with the development of concentration gradients. Due to the sharp transient (Figure 5.3) that we cannot yet interpret, we focus on the analysis of the instantaneous response. We analyse the initial velocity during the first $4.5 \mathrm{~s}$ that provides enough data points to resolve the velocity profile (in comparison to $\sim 50$ s necessary to achieve steady state). As shown in Figure 5.2, close to the entrance of the capillary the particles are not equally spread over the height such that data points are missing for the first $10 \mathrm{~s}$ near the catalyst wall $(z=0)$. Nevertheless, we have sufficient data points to fit the velocity across the height of the capillary which gives us the velocity at the catalyst surface while at the same time determining the diffusio-phoresis of the tracer particles. The kinetics together with the reactant and product mobilities for the three type of surfaces, $\mathrm{TiO}_{2}$, PDMS and PS, were fitted against the experimental $z$ velocity profiles at multiple $y$ locations through an iterative process. The fitted values are presented in Table 5.1. The simulated particle velocity profiles are presented in Figure 5.4 a. and b. against the experimental data. Phoresis of tracer particles has been taken into account for the simulation results. We decouple the reactant and product contributions in Figure 5.5 where we show the diffusio-osmotic velocity together with the individual reactant and product terms from eq. 5.4 employing the mobilities reported in Table 5.1 for both $\mathrm{TiO}_{2}$ and PDMS surfaces along the length of the channel.

In the end, we can vary the driving force for this surface flow by changing the kinetics, as the photocatalytic reaction rate depends on the UV light inten- 
sity [67]. Figure 5.6 shows the experimental particle velocity dependency on the UV light intensity at the PDMS surface and in the middle of the channel. These are the interpolated values based on a quadratic regression of the velocity profile across $z$ between $12 \leq y \leq 32 \mu \mathrm{m}$ for the first 10 seconds. The range has been extended as the triangular profile of the surface velocity is shifted in the $y$ direction depending on the reaction kinetics as the concentration profile is altered. The data for the middle channel is an indication of the surface velocity at the catalyst side. The data at the $\mathrm{TiO}_{2}$ surface is missing as the incoming stream from the large reservoir does not have a homogeneous particle distribution. There is a particle free region close to the bottom of the channel which may be caused by the shear lift force which propels the particles upwards [201]. In the dead-end pore the particles redistribute across the channel due to the phoretic migration towards the active surface (Figure 5.2 ). While the main phoretic component is in the $y$ direction, the phoresis in $z$ direction is still present even if one order of magnitude less. This phoretic velocity will depend on the kinetics. Therefore at lower UV light intensities the particle redistribution inside the capillary is reduced, limiting the number of data points.

The diffusio-osmotic flow at the PDMS and $\mathrm{TiO}_{2}$ surfaces follows a different development with the increase in UV light. The surface flow at the PDMS side levels off sooner than at the catalyst surface which indicates that UV light intensity may affect both the kinetics and the surface charge of $\mathrm{TiO}_{2}$. As the PDMS surface is inactive, a further increase in the velocity at the $\mathrm{TiO}_{2}$ can be explained by an increase in mobility as a result of higher surface charge.

The parameters presented in Table 5.1 do not reveal accurately the transient. To accomplish this, the variation of mobilities with respect to time would be required to accurately fit the experiments. One possibility would entail a nonlinear combination between the reactant and product contributions that would depend on the relative interfacial composition that changes with the degree of conversion, altering the mobilities as the reaction proceeds. Hence, a more detailed approach is required to fully understand the transient. An a priori quantification of the mobilities would entail molecular dynamics simulation with an explicit description of the interaction potentials [45]. A further level of complexity could arise from a change in interaction potential between the chemical species and the catalyst surface under in-situ conditions. We speculate that this is a general inherent mechanism by which a reaction is activated and anticipate that diffusio-osmotic phenomena will become an experimental technique for in-situ active surface characterisation in case of well 


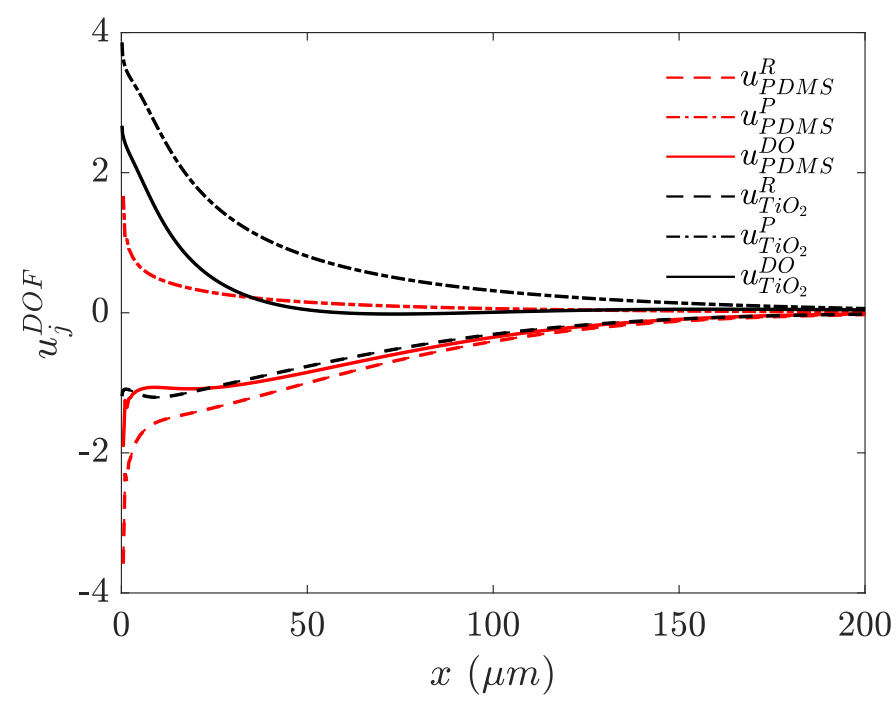

Figure 5.5: Dimensionless diffusio-osmotic velocity together with the reactant and product individual contributions (eq. 5.4 employing the mobilities reported in Table 5.1) for the $\mathrm{TiO}_{2}$ and PDMS surfaces along the length of the channel at $\mathrm{t}=4.5 \mathrm{~s}$. 


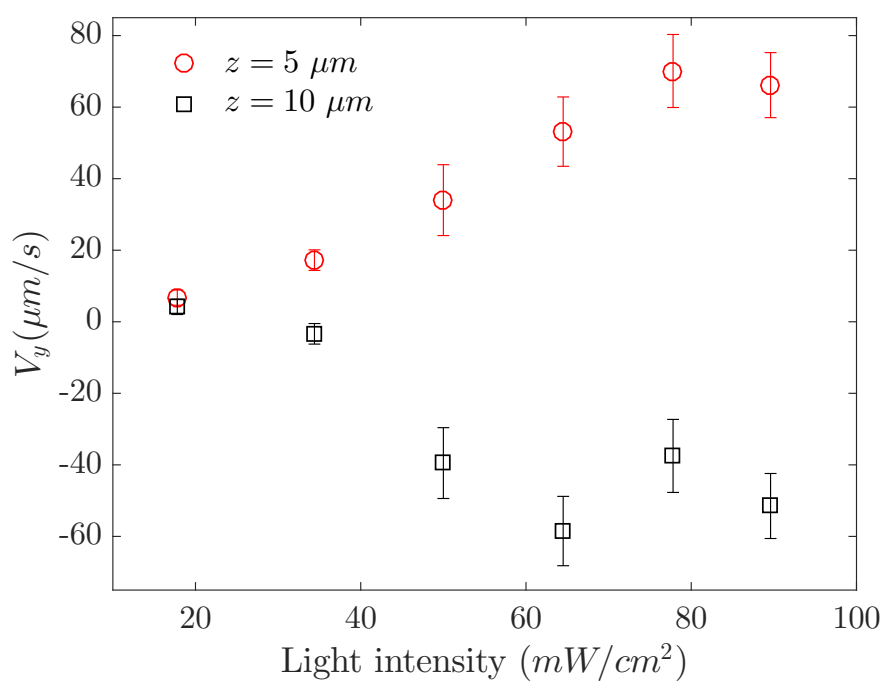

Figure 5.6: a. Particle velocities at the PDMS surface (black markers) and in the middle of the channel $(z=5 \mu m)$ (red markers) for different light intensities.

defined systems (i.e. known reaction mechanism and kinetics for all elementary reactions).

\subsection{Conclusion}

We demonstrate that convective transport is characteristic inside catalytic dead-end pores as a result of generated surface flows, solely. These surface flows are induced by concentration gradients that form during catalysis inside the pores. We quantify and explain the onset of diffusio-osmosis and discuss its relevance in existing catalytic systems. We visualise and quantify the flow in 3D using the General Defocusing Particle Tracking technique. We analyse the phenomena using a model that includes the fluid dynamics actuated by the concentration gradients that arise due to the catalytic reaction. We are able to extract parameters revealing the interaction strength between the reactant/product chemical species and the catalytic surface. In the end, we probe the dependency of this in-situ generated diffusio-osmotic flow on 
its driving force by varying the reaction rate which changes accordingly the concentration gradients of the reactant and product species. The current approach is based on flow in a dead-end pore that has a radius much larger than the interaction length of the chemical species with the surfaces. This work demonstrates the potential of self-induced mixing in dead-end catalytic pores and motivates further studies of different chemistries and surface properties in order to understand how best to utilize it for enhancing catalytic performance. 


\section{6 \\ Catalytically induced flows: Increasing conversion with less catalyst ${ }^{\circ}$}

We illustrate in this paper a simple concept by which patterning a catalyst, i.e. making use of discontinuous patches, generates a diffusio-osmotic flow that mixes the otherwise quiescent liquid near the surface and thereby enhances chemical conversion. The magnitude of this surface flow and the extent to which it impacts mass transfer and consequently catalytic conversion depends foremost on the kinetics of the system. The origin of this flow lies on the concentration gradients generated by the contrast in catalytic surface reactivity. In this study, we use a numerical model employing a diffusio-osmotic slip velocity to explore the role of these surface flows towards enhancing mass transfer. To place the idea of improving conversion by simply redistributing the catalytic material in a general framework, we derive scaling laws which provide guidelines for the dependency of diffusio-osmotic velocity and conversion enhancement on the reaction kinetics and interaction potential between chemical species and the catalytic surface. In the end, we provide criteria for dimensioning the catalyst patch to maximize the benefit of these surface flows.

${ }^{\circ}$ Submitted as: Aura Visan and Rob G. H. Lammertink, Catalytically induced flows: Increasing conversion with less catalyst (2019). 


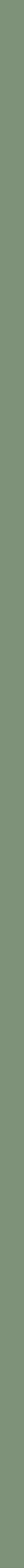




\subsection{Introduction}

There is a constant effort to produce evermore active catalysts. An improvement in the catalyst activity does not necessarily translate into a corresponding increase in chemical conversion. Mass transfer may become limiting very close to the catalyst surface, where diffusion is still the dominating transport mechanism. Mass transfer especially in this near surface region needs to be enhanced in order to benefit from these exceptional materials. One possible approach to enhance near surface transport is to exploit diffusio-osmotic flows. Diffusio-osmotic flows, namely surface flows generated by concentration gradients, were described for the first time in 1947 by Derjaguin [3]. In the presence of macroscopic gradients, osmotic pressure differences develop inside the interfacial layer where the interaction potential spans. The diffuse part of the adsorption layer is mobile, leading to an osmotic flow parallel to the surface. In the case of charged species, an additional driving force comes from the contrast in diffusivity of the corresponding ions which generates an internal electric field. An impressive body of work was dedicated to validate the initial findings and further develop theoretical descriptions [2,4-13, 15-21,30,46, 186-188, 202, 203].

Most of these studies, with the noteworthy exception of Smith and Prieve [10] who investigated the instantaneous rate of deposition of latex particles on a dissolving stainless steel surface through the addition of acid and oxidising agent, relied on externally imposed concentration gradients. Except for particular designs (e.g. pores connecting large reservoirs [30]), immobilised surfaces that pump the solution by diffusio-osmosis would relax the concentration gradients and diminish its own driving force. A sustained driving force requires in-situ generation of concentration gradients.

A few experimental examples of in-situ driven diffusio-osmosis by reactive surfaces $[32,33,40,41]$ have been reported for the purpose of transport in microfluidics. The most famous example concerns the $\mathrm{H}_{2} \mathrm{O}_{2}$ redox decomposition on Pt / Au alternating surfaces [34,36,37,191-194,204]. The uneven distribution of protons leads to a strong electric field which sets in motion the charged Debye layer. The spatially divided consumption and production of protons may benefit from an inherently strong driving force, but concerns only a very specific example relying on "external" electron transport.

Here, we propose a more general method to generate in-situ diffusio-osmotic flows by surface reactivity contrast that is not pinned to a particular chem- 
istry. The contrast in reactivity can be designed by alternating active and inactive regions. We introduce this concept in a new context for diffusioosmosis. That is the possibility of enhancing catalytic conversion by making use of the concentration gradients that are inherent to the design of the system. The synergistic coupling between mass transport and chemical reaction with one enhancing the other could be achieved by a simple redistribution of the catalytic material, i.e. rearrangement of the catalyst into discontinuous patches. In this manner the concentration gradients that are characteristically developing out of plane can now extend in-plane creating the condition for the diffusio-osmotic flow.

The research question that this work tries to answer by means of a numerical model is: Can we impact the conversion capacity of a catalytic system by patterning the catalyst, and if so, what is the dependency of the conversion enhancement on the chemistry of the system, namely reaction rate and interaction potential of the chemical species with the catalytic surface? The model is general in the heterogeneous catalysis framework. We argue that as long as the kinetics are fast enough to place the system in the mass transfer limiting regime, diffusio-osmosis will spontaneously arise for patterned catalysts and can make a significant impact on the overall conversion.

This is an initial paper on the topic that in order to preserve a general framework relies on the simplification of a first order reaction rate where only the reactant species are considered when quantifying the diffusio-osmotic flow. We are aware that more complex mechanistic pathways will deviate from a first order reaction rate and that all chemical species, including intermediates and products will contribute to the overall driving force. While these aspects can be resolved only on a case to case basis, we believe that valuable trends could be drawn from this simple, but more general model.

First, we will illustrate the general features of the diffusio-osmotic flow generated by patterned catalytic surfaces. These include the flow pattern, the transient development of the concentration profile and the enhancement of mass transfer and conversion. In the second part, the characteristic dimensions of the system are investigated, namely the aspect ratio and catalyst coverage. In the last section we explore different reaction kinetics and diffusio-osmotic mobilities (parameter quantifying the interaction potential between the chemical species and the catalytic surface) to identify the potential of the induced flow to impact the conversion. Scaling laws for the surface velocity and Sherwood number $(S h)$ on reaction rate constant and mobility will offer guidelines and quantify the potential of this strategy for enhancing catalytic conversion. 


\subsection{Model}

Momentum and mass transport are solved numerically for a two-dimensional domain of height $L$ and width $2 L$ consisting of a solution of an arbitrary chemical which is consumed at the catalytic surface and replenished by the top reservoir. We work under the premise of a given (stagnant) boundary layer thickness and determine the optimum catalyst pattern design accordingly. The domain is periodic in the $x$ direction. The width represents the period of a repeating patterned surface consisting of alternating active and inactive regions at varying ratios. The domain was chosen for better visualisation in order to encompass 2 vortexes instead of 1 vortex which would represent the basic symmetry of the system, while keeping the aspect ratio of the vortex near unity.

The system is governed by the Stokes and the continuity equations for fluid dynamics where the inertial term is neglected due to small Re numbers and the species conservation equation for mass transport which includes advection and diffusion. In dimensionless form they are given by:

$$
\begin{gathered}
\frac{1}{S c} \frac{\partial \boldsymbol{u}}{\partial t}=-\nabla p+\nabla^{2} \boldsymbol{u} \\
\nabla \cdot \boldsymbol{u}=0 \\
\frac{\partial c}{\partial t}=\nabla \cdot(\nabla c-\boldsymbol{u} c)
\end{gathered}
$$

The Schmidt number is $S c=\nu / D_{0}$ where $\nu$ is the kinematic viscosity, $t=$ $\tilde{t} / t_{0}$ where $t_{0}=L^{2} / D_{0}$ is the diffusion time with $D_{0}=4.6 \times 10^{-10} \mathrm{~m}^{2} / \mathrm{s}$ the diffusivity of methylene blue [205] and $p=\widetilde{p} /\left(\mu D_{0} / L^{2}\right)$ is the pressure. The dimensionless velocity $\boldsymbol{u}$ is obtained with respect to the diffusion velocity $\boldsymbol{u}=\widetilde{\boldsymbol{u}} L / D_{0}$, where the height of the boundary layer is $L=500 \times 10^{-6} \mathrm{~m}$. $c=\widetilde{c} / c_{0}$ is the dimensionless concentration, where $c_{0}$ is the bulk concentration which sets the top boundary condition.

The upper boundary condition is set to full slip in the $x$ direction and no velocity in the $y$ direction, to prevent formation of a viscous layer, unless 
stated otherwise. The active and inactive regions of the solid surface are defined by the diffusio-osmotic slip, $u_{D O}$.

$$
u_{D O}=-\frac{\mu}{c} \frac{\partial c}{\partial x}
$$

The mobility $\mu$ is the prefactor of the diffusio-osmotic slip which lumps the effects of the diffusion potential and osmotic pressure gradient set by the interaction potential between reactant molecules and the catalyst surface. This can be quantified via the zeta potential in case of charged species or a more general integrated interfacial concentration $[2,5,6]$. We reference the mobility to the diffusivity of the solute, $\mu=\widetilde{\mu} / D_{0}$.

$$
\widetilde{\mu}=-\frac{\varepsilon k_{B} T}{\eta e}\left(\beta \zeta+\frac{4 k_{B} T}{e} \ln \left(\cosh \left(\frac{e \zeta}{4 k_{B} T}\right)\right)\right)
$$

where $\varepsilon$ is the permittivity of water, $k_{B}$ the Boltzmann constant, $T$ the temperature, $\eta$ the viscosity of water, $e$ the elementary charge, $\zeta$ the zeta potential and the diffusivity contrast $\beta=\left(D_{+}-D_{-}\right) /\left(D_{+}+D_{-}\right)$with $D_{+}=$ $4.3 \times 10^{-10} \mathrm{~m}^{2} / \mathrm{s}$ and $D_{-}=2.03 \times 10^{-9} \mathrm{~m}^{2} / \mathrm{s}$ the cation and anion diffusivities $[206]$.

For the species transport, the reservoir boundary condition is defined by a fixed bulk concentration, $c=1$. The boundary condition for catalyst surface is represented by the normalised surface flux which is a pseudo-homogeneous first order reaction rate, $\frac{\partial c}{\partial y}=-D a_{I I} c$, where $D a_{I I}=k L / D_{0}$ is the dimensionless second Damköhler number, with reaction rate constant $k$. The inactive region has a no flux boundary condition, $\frac{\partial c}{\partial y}=0$. The left and right boundaries are set as periodic, with equal concentration and flux for each boundary. The governing equations are solved using Finite Element Analysis in COMSOL Multiphysics 5.3 with a relative tolerance of 0.05 . P2 + P1 discretization (second-order Lagrange elements for velocity and first-order elements for pressure) was used to solve the Stokes equations and second-order Lagrange elements for the species conservation equation. The simulations were performed using an unstructured mesh with triangular elements. The mesh was refined near the walls and intersection points and independency was checked. The complete mesh consisted of approximately 180,000 domain elements.

We chose a catalytic example from literature to showcase the induced flow conversion enhancement. Yang et al. reports the photocatalytic degradation 
a.

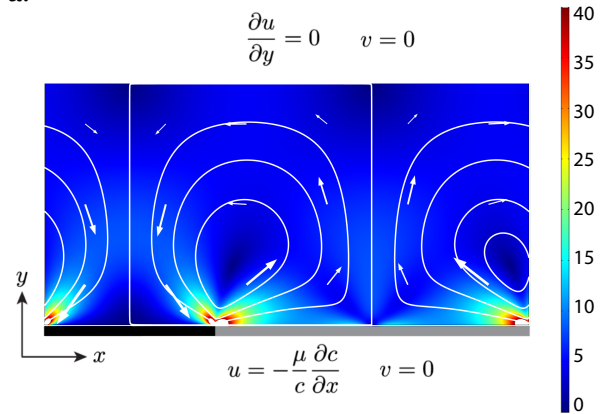

c.

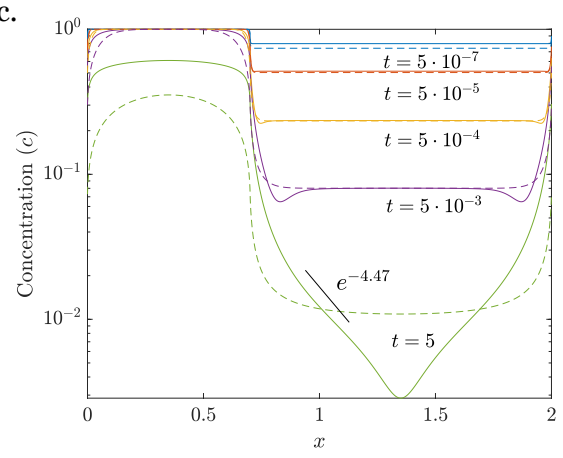

b.

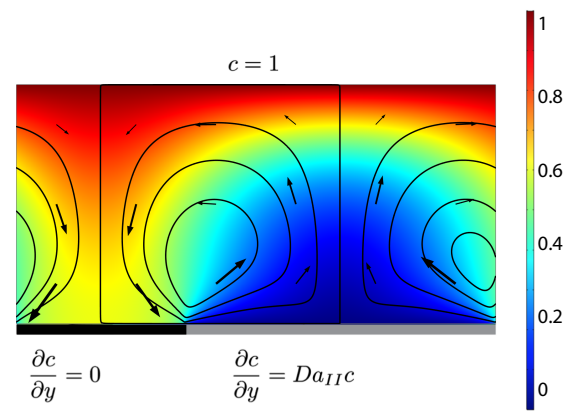

d.

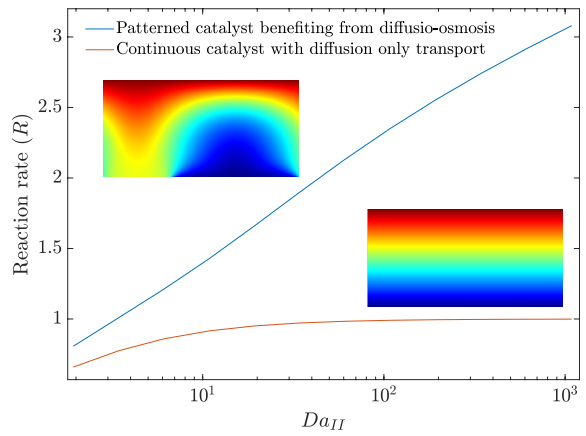

Figure 6.1: For all graphs $\mu=2.82, D a_{I I}=98, \omega=0.65$, except for parametric sweep a. Absolute velocity map at steady state, $|\boldsymbol{u}|=\sqrt{u^{2}+v^{2}}$ with streamlines and velocity vector arrows. Boundary conditions for the momentum equation are indicated. The grey surface region represents the active region, while the black corresponds to the inactive surface part. b. Concentration map at steady state with fluid flow streamlines and velocity vector arrows. Boundary conditions for the species conservation equation are indicated. c. Transient surface concentration profiles for an active region $0.7<x<2$; continuous lines for a diffusio-osmosis scenario, dotted lines for a fictitious diffusion only scenario d. Surface averaged reaction rate at steady state $\left(R=\left.D a_{I I} \int_{2-2 \omega}^{2} c \cdot d x\right|_{y=0}\right)$ vs surface reactivity $D a_{I I}$. For a continuous catalyst surface (dotted red line) the conversion levels off for fast kinetics while a discontinuous catalyst surface induces diffusio-osmotic flow (continuous blue line) which extends the conversion capacity for higher catalytic activity. 
of methylene blue by poly-o-phenylenediamine modified $\mathrm{TiO}_{2}$ with $\mathrm{pH}$ dependent kinetics that vary between $k=5.5 \times 10^{-5}$ and $1.9 \times 10^{-4} \mathrm{~m} / \mathrm{s}$ [207]. The $\mathrm{pH}$ dependent zeta potential of $\mathrm{TiO}_{2}$ is reported between $\zeta=100$ and $\zeta=-150 \mathrm{mV}$ [208]. To avoid any bias we select an intermediary value for kinetics $\left(k=9 \times 10^{-5} \mathrm{~m} / \mathrm{s}\right)$ and an average zeta potential $(\zeta=-75 \mathrm{mV})$ that is characteristic for metal oxides. The diffusivity contrast gives a $\beta=-0.65$ which sets the mobility to $\widetilde{\mu}=1.298 \times 10^{-9} \mathrm{~m}^{2} / \mathrm{s}$ and the dimensionless analogue to $\mu=2.82$. The optimised characteristic dimensions (aspect ratio 1:2 and $65 \%$ catalyst coverage) will be rationalised later on. The further studies preserve these characteristics while varying one parameter at a time. The example concerns realistic values for the mentioned parameters to benchmark the effect, and in our study we report a range around these values to investigate their relative influence.

\subsection{Results and discussion}

As the reaction proceeds and the depletion zone forms, the solute influx leads to significant gradients along the surface which initiates the diffusio-osmotic flow (Fig. 6.1a and b). Initially, the gradient is contained at the inactive/active intersection. Once the flow sets in, it transports fresh solution towards the catalyst, extending the gradient which intensifies the flow. Due to the symmetry of the system, diffusio-osmotic slip is generated in both directions. The inward flows extend half way across the catalyst and diverge each other upwards giving rise to two mirrored vortex structures.

Intuitively, one would imagine that replenishing the surface of the catalyst with fresh solution will diminish the concentration gradient such that the diffusio-osmotic slip will limit its own driving force. However, comparing the transient concentration profiles for the slip and no slip scenarios, there is an obvious reinforcement in the gradient development. Figure 1c presents the surface concentration profiles for a scenario where the surface flow is absent $\left(u_{D O}=0\right.$, dashed lines) and where it is present due to the diffusio-osmotic flow $\left(u_{D O}=-\mu \frac{1}{c} \frac{\partial c}{\partial x}\right.$, continuous lines). The profiles illustrate the self-enhancing effect of the flow parallel to the surface to its own driving force, namely the concentration gradient. The steeper gradients are a result of the flow along the active surface which introduces a residence time distribution. We tried to describe this effect by an advection-reaction mass balance (Eq 6.6) just along the catalyst surface. This corresponds to a $1 \mathrm{D}$ model where the origin 
of the flow lies only in the concentration gradient generated by the reaction, disregarding the reactivity contrast and the replenishment of the boundary layer by the 2D diffusional and advective transport. The equation represents a solute mass balance which assumes a steady state between the consumption of the solute by the surface and a diffusio-osmotic flow along this surface.

$$
\frac{d}{d x}\left(u_{D O} \cdot c-D a_{I I} \cdot c\right)=0
$$

The diffusio-osmotic flow $u_{D O}$ is a function of the concentration and its gradient (Eq 6.4), and hence a function of $x$. Using Eq 6.4 we obtain the following expression for the surface concentration along the half catalyst patch:

$$
c=-\frac{c_{\omega}-c_{0} \cdot e^{-\frac{D a_{I I}}{\mu} \omega}+\left(c_{0}-c_{\omega}\right) \cdot e^{-\frac{D a_{I I}}{\mu} x}}{e^{-\frac{D a_{I I}}{\mu} \omega}-1}
$$

where $c_{0}$ is the concentration at the start of the active domain, and $c_{\omega}$ is the concentration at the center of the active domain of length $2 \omega$.

The accuracy of this analytical simplification diverges as the reaction kinetics increase and the corresponding increase in diffusional transport as well as the advective transport provided by the circular flow relaxes the expected concentration gradients. This is also illustrated in Fig. $2 \mathrm{~b}$ where the linearity between $u_{D O}$ and $D a_{I I}$ breaks down around $D a_{I I} \sim 10^{1}$. It is not surprising that for $D a_{I I}=98$ corresponding to the show case, the dominating slope of the logarithmic fit to the numerical data, -4.47 (inverse decay length), is significantly lower than the analytical value for $-\frac{D a_{I I}}{\mu}=-34.7$ which dominates the slope of Eq. 6.7 for $c>c_{\omega}$. This simple model illustrates that while DO flow is initiated by the contrast in reactivity, it develops because of the increase in residence time along the catalyst which generates a constant normalised gradient given by $-\frac{D a_{I I}}{\mu}$. For a first order reaction rate we would expect a constant DO velocity equal to $D a_{I I}$. This original driving force is significantly decreased as concentration gradients are relaxed by transport in the wall-normal direction.

In addition to the residence time distribution, the gradient is accentuated at the intersection through the fresh solution supply that covers the entire inactive region. Fig. 1b illustrates the higher concentration at the inactive side. The flow accelerates again before being diverged midway into the catalyst patch due to the stagnation point. Here, the concentration is the lowest due to the longest local residence time. 
The driving force for this diffusio-osmotic flow lies in the mass transfer limitation, i.e. the concentration gradients that develop due to the interplay between reaction and mass transfer rates. For higher consumption rates, these gradients will deepen and consequently increase the diffusio-osmotic flow. This increased convective transport will have a higher impact on the concentration profile which will translate into a corresponding increase in the conversion enhancement. While for a continuous catalyst where diffusion is the only transport mechanism, the conversion levels off at $D a_{I I} \geq 10$ due to increasing mass transfer limitations, the diffusio-osmotic flow extends the conversion to much higher $D a_{I I}$ numbers. Fig. 6.1d. makes a comparison between the current situation where continuous layers of catalyst are used to the design proposed by this paper which despite using less material can achieve more conversion. Diffusio-osmosis is a self-regulating mechanism with an amplification set by the mobility.

Diffusio-osmosis speeds up the replenishment of the depleted solution thanks to the circular structure of the flow. The mass balance follows two main repeating steps; consumption at the catalyst surface and replenishment at the top boundary. The enhanced mass transport due to mixing in the boundary layer maintains a higher concentration at the catalyst surface which increases linearly the first order reaction rate leading to a higher conversion capacity. The impact of the diffusio-osmotic flow on mass transport depends on the size of the vortex region with respect to the thickness of the diffusion dominated boundary layer. It follows that the vortex width should match this height, so it can reach the reservoir and replenish the catalyst surface with fresh solution (Fig. 6.1a). When the velocity extends throughout the whole boundary layer, it provides the highest degree of mixing. As it became obvious from figure 6.1 already, the vortex is half the size of the catalyst pattern due to the symmetry of the system. The domain chosen for better visualisation encompasses 2 vortexes (instead of 1 vortex representing the basic symmetry of the system) which gives a 1 to 2 height to width optimal ratio.

Local strong relative concentration gradients can sustain significant diffusioosmotic velocities regardless of small mobilities. As shown in Fig. $6.2 \mathrm{~b}$ for $\mu=2.82$, the average catalyst surface velocity can easily surpass one order of magnitude for faster kinetics. However, as discussed previously, the velocity decreases along the catalyst patch as diffusion relaxes the concentration gradients. It follows that the catalyst patch has to be dimensioned such that the contrast in reactivity regenerates these gradients when this velocity becomes inefficient. The relevance of the diffusio-osmotic flow is preserved as long as it 
exceeds the diffusion velocity, $-\mu \frac{1}{c} \frac{\partial c}{\partial x}>u_{\text {diff }}$. In the framework of preserving in-plane concentration gradients, the diffusion velocity is defined with respect to the inactive patch length, $1-\omega$. We make an arbitrary choice that the threshold for the velocity decrease to be $-\frac{1}{c} \frac{\partial c}{\partial x}=1$ or $u_{D O}=\mu$. The formal criterion becomes $\omega=(\mu-1) / \mu$, where $\omega$ is the dimensionless normalised catalyst patch length which is denoted as catalyst coverage when expressed in percentages. For the parameters used in the showcase, this simple criterion would prescribe an optimum of $64.5 \%$ catalyst coverage, which is identical to the numerically determined optimum value rounded up at $65 \%$. A significant enhancement in conversion of more than $50 \%$ can be generated by the diffusioosmotic slip for $65 \%$ of the catalyst in comparison to a continuous layer (Fig. 2a). For the same amount of catalyst as in a continuous layer, the conversion enhancement exceeds $130 \%$ as can be read in Fig. 6.2d for $D a_{I I}=98$, corresponding to the kinetics used for the showcase.

While slower kinetics will have less impact, it is worth mentioning that this criterion is based on the assumption that $D a_{I I}>\mu$ which corresponds to analytically derived relative gradient originating from the residence time distribution, $-\frac{1}{c} \frac{\partial c}{\partial x}=D a_{I I} / \mu$. Except for localised gradients at the active-inactive junction, this value can be regarded as the maximum driving force that gets relaxed by diffusion. An important observation here is that for slower kinetics, the impact of diffusion on the concentration gradients is minimal, such that the departure from this analytical velocity is insignificant and does not represent the limiting factor. However, if $D a_{I I} / \mu>1$, this starting relative gradient will already be smaller than 1 , which imposes a different criterion. Please note that diffusio-osmosis may still be relevant if mobilities are very high. If we consider this analytical velocity based on the residence time distribution, namely $u_{D O}=D a_{I I}$, the condition for diffusio-osmosis is given by the same comparison to the diffusion velocity, $D a_{I I}>u_{\text {diff }}$, which results in the formal criterion $\omega=\left(D a_{I I}-1\right) / D a_{I I}$.

In order to motivate the use of this approach we retrieved simple scaling laws for the diffusio-osmotic velocity and conversion enhancement based on the kinetics, $D a_{I I}$, and mobility, $\mu$. The driving force for the diffusio-osmotic slip is the relative concentration gradient which is generated by the contrast in reactivity. Higher kinetics will deepen this gradient and increase the velocity accordingly. A linear scaling relation could be inferred between surface velocity and reaction rate constant using a simple convection reaction mass balance along the catalyst (Eq. 6.6). While this is generally valid for the intersection velocity, the average velocity follows this scaling up to $D a_{I I}$ numbers of ap- 
a.

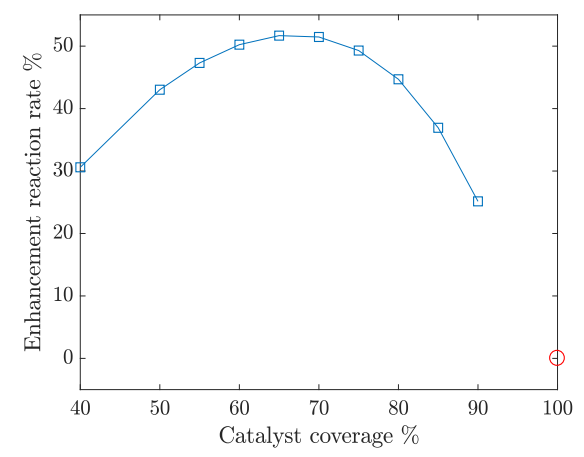

c.

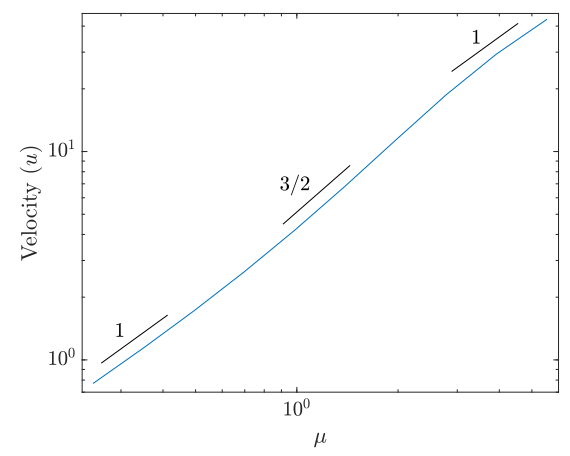

b.

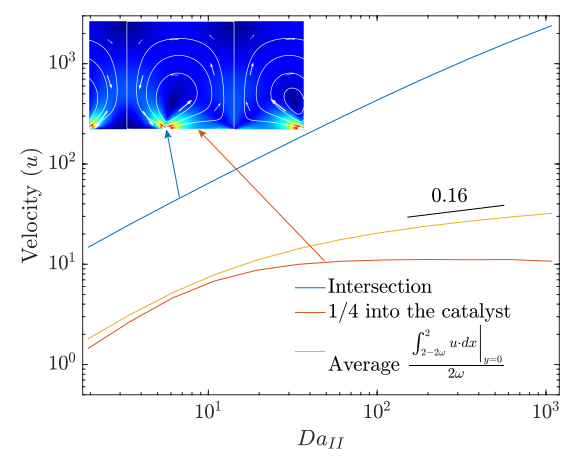

d.

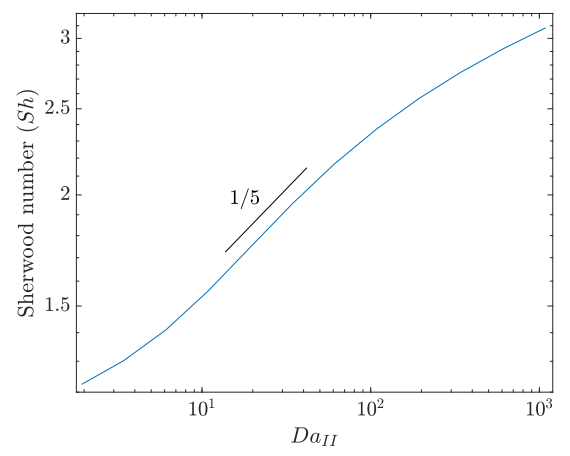

Figure 6.2: All graphs represent the steady state with $\mu=2.82, D a_{I I}=98$, $\omega=0.65$ except for parametric sweeps a. Enhancement in reaction rate, $(S h-$ $1) \cdot 100$, generated by the diffusio-osmotic flow with respect to a diffusion only scenario representative for a continuous catalyst configuration vs. catalyst coverage (catalyst patch / repeating unit) b. Surface induced velocity $u$ vs surface reactivity $D a_{I I}$ c. Surface velocity $u$ vs mobility $\mu$ d. Dimensionless mass transport number $S h$ vs surface reactivity $D a_{I I}$. 
proximately 10 (Fig. 6.2b), after which it proceeds to decrease asymptotically. As discussed previously, for very fast kinetics, the depleted surface enhances transversal diffusion which relaxes the concentration gradients and decreases the velocity. Further along the catalyst surface as shown in Figure $6.2 \mathrm{~b}$ at location $1 / 4$ into the patch, the velocity stagnates for increasing $D a_{I I}$ because increasing kinetics do not translate into an increase in the relative concentration gradient. Beside diffusion, there is an increasing advective contribution coming from the faster diffusio-osmotic flow. The linear increase in velocity cannot be sustained at higher consumption rates as a result of a matching increase in mass transfer. This equilibrium between consumption and replenishment fluxes is also illustrated by the asymptotic decay of both surface and volume average concentrations in this second regime of fast kinetics.

The specific chemistry influences the dynamics of the system not only through kinetics, but also through the interaction potential between the chemical species and the catalyst surface, i.e. the mobility. By scanning different mobilities, three different regimes can be identified (Fig. 6.2c). In the diffusion controlled regime, the diffusio-osmotic slip, $u_{D O}=-\mu \frac{1}{c} \frac{\partial c}{\partial x}$, has a linear dependency on the mobility, $\mu$, given that the low velocity comparable to the diffusion velocity does not significantly impact the concentration profile along the catalyst, i.e $\frac{1}{c} \frac{\partial c}{\partial x}$. The mechanism of the diffusio-osmotic flow in this regime relies only on the reactivity contrast without benefiting from the selfenhancing effect coming from the residence time distribution, as the in-plane gradients relax due to diffusion. The residence time distribution effect of the surface flow kicks in when $\mu$ approaches 1 . In this regime, when increasing the mobility, the velocity couples to its own driving force, because the higher the surface flow, the higher the discrepancy with respect to the diffusion time scale which prevents the decrease in concentration gradients for a given $D a_{I I}$ and thus reinforcing itself. This produces an exponent of $3 / 2$ for the intermediate regime. For mobilities much higher than 1, the exponent decreases back to 1 . In this advection regime, the driving force stagnates because the mixing of the boundary layer at this high velocities limits the further increase in concentration gradients for a given $D a_{I I}$, that is, the efficient replenishment of the catalyst surface is not supported by faster consumption.

The overall increase in mass transfer due to diffusio-osmotic flow with respect to a diffusion only scenario can be evaluated by the ratio between the catalyst surface concentrations for the two different scenarios. The ratio represents the convective to diffusive mass transport rate, i.e. the Sherwood number. 


$$
S h=\frac{\left.\int_{2-2 \omega}^{2} c \cdot d x\right|_{y=0}, u_{D O}=-\mu \frac{1}{c} \frac{\partial c}{\partial x}}{\left.\int_{2-2 \omega}^{2} c \cdot d x\right|_{y=0}, u_{D O}=0}
$$

For laminar flow parallel to a flat surface there is a well known mass transfer correlation, $S h=0.664 R e^{1 / 2} S c^{1 / 3}$ [209]. The fitting $S h$ vs $R e^{1 / 2}$ of the numerical data gave a prefactor of $S h /\left(R e^{1 / 2} S c^{1 / 3}\right)=2.4$. This represents a mean $S h$ number almost 4 (3.62) times higher than for the parallel laminar flow, suggesting further enhancement by the vortex structure of the diffusioosmotic flow.

In the end we can directly infer the flux enhancement from the chemistry of the catalytic system. The convective transport extends the conversion capacity of the system following a scaling of the $S h$ number with $D a_{I I}^{1 / 5}$ (Fig. 2d) up to $D a_{I I}$ of 100 . Beyond that, the exponent decays in an ironic development due to the self-limiting effect of the increasingly faster mass transport which relaxes the concentration gradients and limits its own driving force.

We want to make a final note regarding the configuration of the system. Until now, we have described the system as a diffusion dominated boundary layer close to a catalytic surface. By a common engineering practice this boundary layer is considered stagnant while encompassing all bulk mass transfer resistances such that the bulk solution at the top boundary is considered perfectly mixed. We made this decision to preserve a general framework. Any choice regarding external flow would have introduced a bias in the outcome. However, we want make a few qualitative remarks. For the given 2D model, an external laminar flow parallel to the catalyst surface can be considered in the $x$ and $z$ directions, that is either a transversal or longitudinal flow with respect to the pattern orientation.

Regarding the transversal arrangement, simulations showed that the results reiterate with small deviations as long as the magnitude of the external flow does not surpass the local diffusio-osmotic velocity. For higher external velocities, the enhancement decreases accordingly. The unidirectional external flow breaks down the symmetry of the vortexes, such that the depleted layer does not reach the top boundary where can it get replenished, but it is entrained by the external flow and changes to a tangential configuration. Approximately half of the average surface velocity is preserved even for very intense external flows in comparison to a stagnant layer scenario. In this case, the diffusioosmotic velocity is contained at the active/inactive intersection. Instead of 


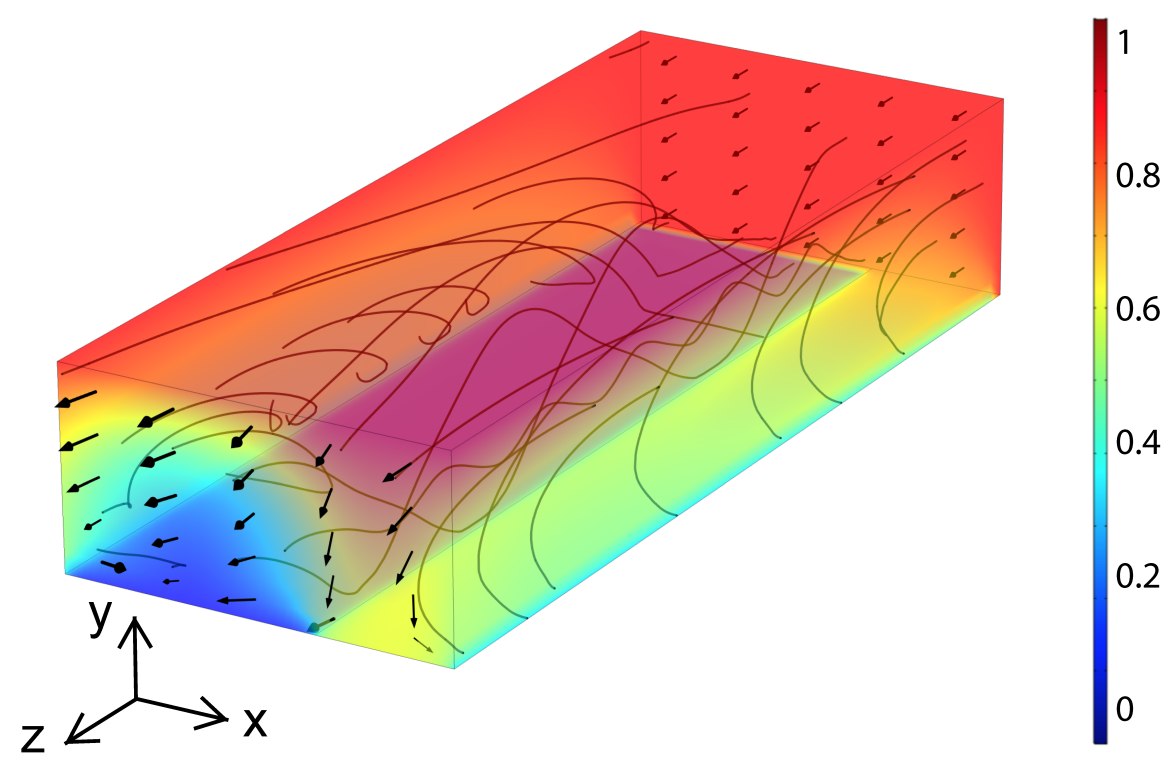

Figure 6.3: 3D concentration map at steady state with fluid flow streamlines and velocity vector arrows corresponding to the show case, $\mu=2.82, D a_{I I}=$ $98, \omega=0.65$, under an external unidirectional flow along the catalyst patches, i.e. in the $z$ direction, of magnitude $w=10$. The in-situ reaction driven surface flow that develops in the transverse direction leads to the helical profile.

extending further into the patch, the DO flow is picked up by the external flow. This localised surface velocity has hardly any contribution to mass transfer, indicating that the $y$ component of the velocity field is required to ensure the by-passed replenishment of the depleted layer.

Regarding the longitudinal arrangement, preliminary 3D simulations (Figure 6.3) showed a mirrored helical flow as a result of the laminar flow which introduces a residence time distribution across the height of the channel. While this $3 \mathrm{D}$ flow structure changes the results, there are no foreseeable limitations as for the previous configuration which indicates an optimum pattern orientation with respect to a unidirectional external flow. 


\subsection{Conclusion}

We propose a new context for reaction induced diffusio-osmosis, i.e enhancing chemical conversion by the geometric patterning of the catalyst. The reactivity contrast designed by alternating active and inactive regions spontaneously generate in-plane gradients that drive a steady diffusio-osmotic flow. While the details of the numerical model may need to be adjusted for particular catalytic reactions, the approach is not pinned to a certain chemistry. Concentration gradients will develop as long as kinetics are fast enough to place the system in the mass transfer limiting regime. The diffusio-osmotic flow is redirected out of plane due to the symmetry of the system and mixes the otherwise diffusion dominated boundary layer which greatly enhances mass transport and thus impacts the overall conversion.

We use a numerical model based on a first order reaction rate assumption to study the structure of the diffusio-osmotic flow, its development and dependency on the catalytic chemistry, i.e. reaction kinetics and interaction potential between chemical species and catalytic surface (expressed through the mobility parameter). The study reveals that while diffusio-osmosis is initiated by the contrast in reactivity, it develops along the catalyst due to a self-reinforcing mechanism specific to active surfaces. The flow parallel to the catalyst surface introduces a residence time distribution and thus a concentration gradient which is the sustaining driving force that depends on both kinetics, $D a_{I I}$ and mobility, $\mu$. Based on this fundamental understanding we introduce criteria to dimension the catalyst patch according to the chemistry. Scaling laws provide a direct correlation between the catalytic chemistry, the dynamics of the system and the conversion enhancement. Specific chemistries, with known reaction kinetics and interaction potential (i.e. mobilities), can now be tested against this predictive model. 


\section{7 \\ Reaction induced diffusio-phoresis of ordinary catalytic particles ${ }^{\circ}$}

In this paper we demonstrate experimentally diffusio-phoresis of plain catalytic particles driven by the macroscopic concentration field that the particles generate by themselves. This introduces a general framework for heterogeneous catalysis where the driving force relies on solute gradients that are generated by a mere uneven distribution of catalytic particles. Whenever an inhomogeneity appears in the particle distribution, the higher particle density region will lower the reactant concentration more than the surrounding environment. This macroscopic concentration gradient propels the particles towards higher reactant concentration by surface driven flows. The nature of this flow originates from osmotic pressure differences and diffusion potentials in the case of charges species. This context of diffusio-phoresis demonstrates an additional transport mechanism for slurry reactors. We investigate the reaction induced particle movement for a photocatalytic degradation reaction using $\mathrm{TiO}_{2}$ suspensions in a co-flow microchannel. We have analyzed the experimental particle distribution by means of a numerical model that includes diffusio-phoretic movement of the catalytic particles. This model stresses the importance of particle characteristics, like surface potential, reactant and product interaction, and reaction kinetics with respect to the catalyst particle migration.

${ }^{\circ}$ Submitted as: Aura Visan and Rob G. H. Lammertink, Reaction induced diffusio-phoresis of ordinary catalytic particles (2019). 


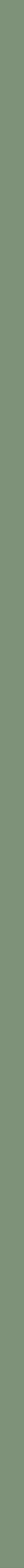




\subsection{Introduction}

Surface flows generated by concentration gradients and their particle propulsion analogue have been introduced by Derjaguin under the name of capillary osmosis and diffusio-phoresis. The phenomenon was speculated theoretically, starting from an extensive knowledge on surface science. Derjaguin rationalized that due to molecular interactions between solute and solid surface, a solution has a different chemical composition close to that solid surface which is susceptible to bulk concentration gradients. A gradient in osmotic pressure will develop within the interfacial layer where the interaction potential spans. Given that the diffusive part of the adsorption layer is mobile, it drives an osmotic flow parallel to the surface $[2,3]$. Suspended particles will be propelled by such a surface flow in a solute concentration gradient. This propulsion mechanism is analogue to particle electro-phoresis in an external potential gradient. Derjaguin illustrated the theory regarding particle migration under electrolyte concentration gradients using an industrial example, namely the ionic deposition of latex particles under the dissolution of an electrolyte coating [4].

Following Derjaguin, Prieve and Anderson extended the theory in a series of papers. They derived the migration velocity of particles in gradients of neutral species while including other interaction potentials than the exponential profiles [5]. They went on to prove that diffusio-phoresis in case of finite double layers will depend on the particle size [6]. They corrected for the effect of strongly adsorbing solutes which affect the external concentration field [7]. They also studied the effect of arbitrary distribution of zeta potential across the surface of the particle [8] and, later on, they considered both non-spherical and non-uniformly charged particles with finite double layers [9].

The theory was soon after validated in a series of experimental papers [1013, 15-19, 187, 188]. Lechnick and Shaeiwitz accounted for the dependency of the zeta potential on the electrolyte concentration [13]. Staffeld and Quinn inferred the particle-solute interaction potential by observing the diffusiophoresis in gradients of neutral polymers (Dextran) and charged hard spheres (Percoll) [18]. More than three decades later, Shin et al. experimentally probed the effect of the finite Debye layer thickness and confirmed the theory of Prieve on the particle size dependent velocity [14]. Paustian et al. looked into the particle migration under a gradient of solvent composition. They used hydrogel membranes to divide channels with different composition and mon- 
itored the translation of particles after stopping the flow [20]. Nery-Azevedo et al. used the same device for tracking the migration of particles exposed to ionic surfactant gradients [21] and Shi et al. used it under opposing gradients of different electrolytes [22] where the neutralizing reaction leads to focusing of particles.

The examples mentioned above focused on the fundamental aspects of diffusiophoresis, probing the chemical nature of the gradient with various solid-solute molecular interactions. These experimental endeavors served initially to confirm the theory and later on use it to infer surface properties and solute wall interactions. This increasing confidence in the theory helped transition to various applications. Shin et al. developed a very simple, but elegant experimental design using a dead-end channel to measure the zeta potential of walls and particles simultaneously $[27,189]$. Due to the well-defined electrostatic interactions, one can use a plain electrolyte such as sodium chloride to infer the charge density of various surfaces by visualizing collective particle dynamics. Particle focusing and separation based on zeta potential was achieved by meticulous design of concentration profiles inside microchannels [28, 29]. Oil recovery can be enhanced by diffusio-phoresis of oil droplets out of the dead-end pores [26] and membrane fouling due to particle deposition could be reduced by adding sodium carbonate particles, that by dissolving counteract the diffusion potential that appears due to the inherent salt rejection [23-25]. Other applications using in-situ gradient generation involve ion-exchange surfaces. Niu et al. uses ion-exchange particles for cargo transport [210] while Florea et al. suggests an antifouling material application as a result of the migration of particles away from a Nafion ion-exchange membrane [190].

There are two types of reaction induced phoretic phenomena that have been studied so far. The first is chemotaxis in biological systems where cells mostly react to the external composition. Their low activity has a small impact on the surrounding environment, such that the reactivity feedback on their dynamics is of lesser degree. The migration of cells towards higher nutrient sites is studied under externally imposed gradients while trying to decouple the contribution brought by their own activity [211-214]. The second example is the migration of bimetallic particles that catalyze complementary redox reactions which leads to an uneven distribution of protons and hence an external electric field which electrophoretically drives the particles. This chemistry involves the production of charged species that are not being screened by counter-ions thanks to the "external" electron transport through the bimetallic particle. This phenomenon is based on spontaneous electrochemical reactions using 


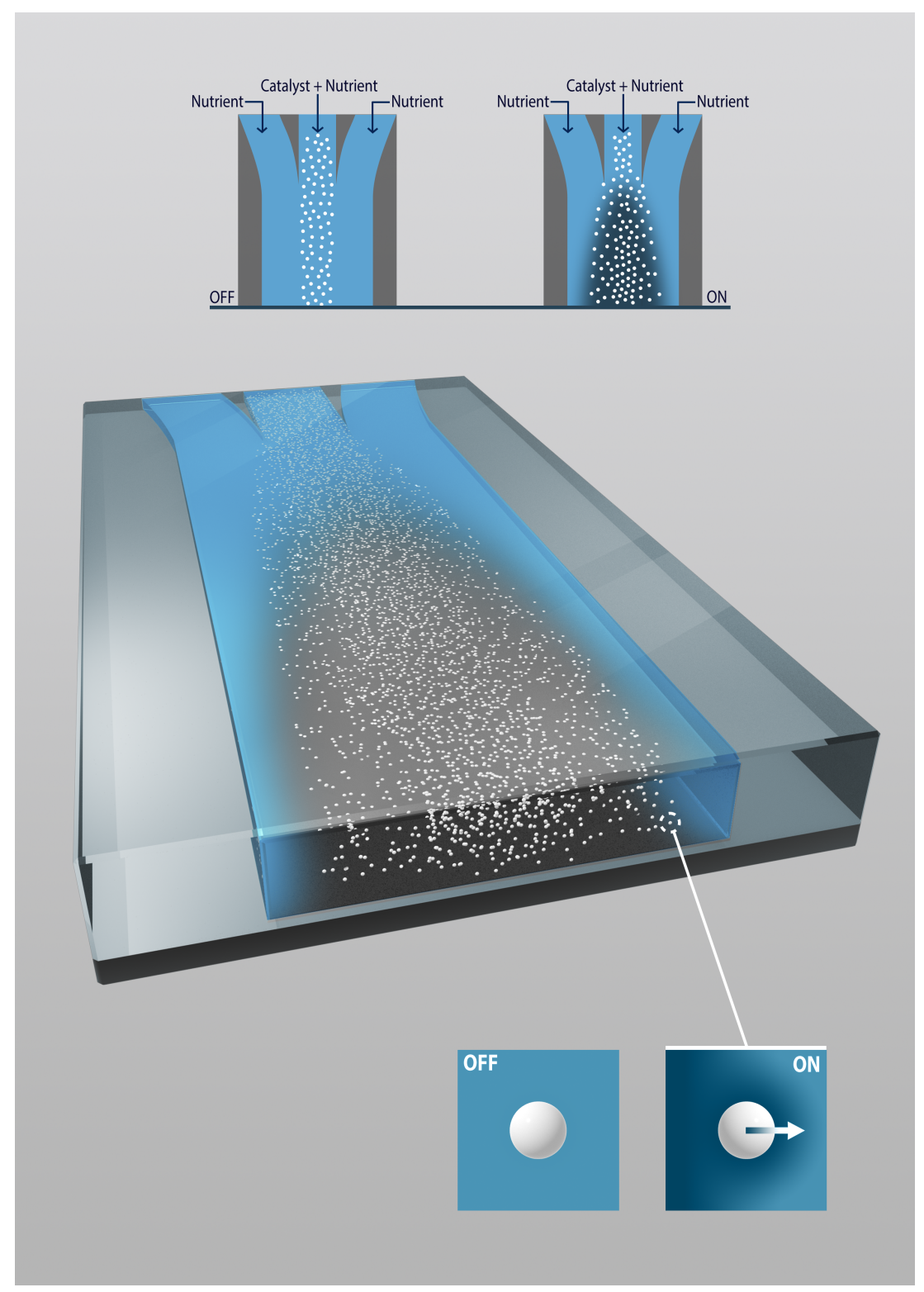

Figure 7.1: Sketch of the setup. In a coflow microchannel, reactant is added to each inlet, while photocatalytic particles are only present in the middle stream. This uneven particle distribution leads to reactant concentration gradients during photocatalytic degradation. These concentration gradients drive catalytic particles via diffusio-phoresis. 
particular chemicals, namely hydrogen peroxide or hydrazine, and Janus-type particles to provide the corresponding transport [34-39].

In this paper, the driving force relies on solute gradients generated by the inhomogeneous distribution of otherwise homogeneous catalytic particles (Figure 7.1). The spontaneously occurring surface flow can have a great impact on the overall conversion, as interfacial transport is a known limitation for heterogeneous catalysis. Reactant and product gradients are generated by an uneven distribution of catalytic particles, rather than by asymmetric particles (Janus type). The catalytic reactant consumption creates exceptional steep gradients. There is a synergy between the surface flow and the reaction rate with one enhancing the other that is particularly exciting.

The study describes the diffusio-phoresis of photocatalytic particles that catalytically generate macroscopic gradients caused by the uneven distribution of the particles. The migration of photocatalytic particles is studied systematically in a channel where an aqueous solution of an organic contaminant, methylthioninium chloride, is contacted under continuous flow with a particle suspension containing the same solute concentration. When UV light is turned on, the photocatalytic particles decompose the contaminant lowering its concentration inside the colloidal stream. The gradient in concentration that is photocatalytically generated leads to the migration of particles toward the higher concentration site. The effect of the reaction rate on the migration of particles is evaluated by changing both the light intensity and initial particle concentration. We analyze this experimentally observed migration mechanism by numerical simulations.

\subsection{Methods}

\subsubsection{Experimental}

Methylthioninium chloride, also known as methylene blue (MB) and Tris buffer were purchased from Sigma Aldrich and $\mathrm{TiO}_{2}$ suspension from Evonik. The particle agglomerates were measured to be $157 \pm 70 \mathrm{~nm}$ in size. The Si-glass chip has been fabricated in a cleanroom using standard lithography, DRIE etching of silicon and anodic bonding. The channel has 55x600 $\mu \mathrm{m}$ crosssection (height $\mathrm{x}$ width) and is $10 \mathrm{~mm}$ in length. The design was inspired 

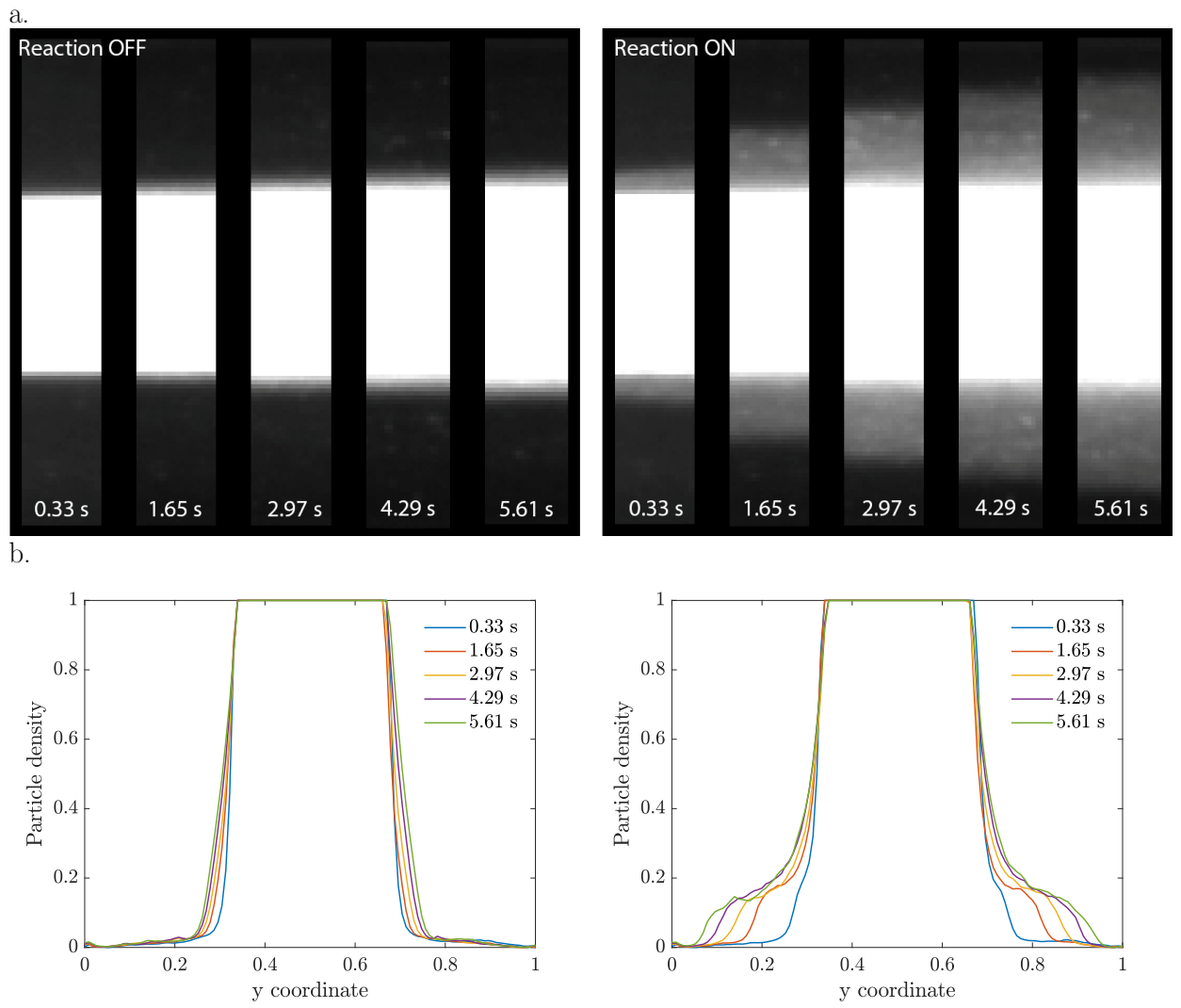

Figure 7.2: 2D and 1D particle density profiles for various residence times. (a) optical microscopy images of the particle distribution at different positions in the downstream direction, corresponding to the indicated residence time. Light off (left), on (right), $50 \mu \mathrm{M} \mathrm{MB}, 1 \mathrm{wt} \% \mathrm{TiO}_{2}$. (b) particle density profiles obtained from the microscopy images. Little diffusion is observed for the situation without catalytic reaction, while a broader shoulder of particles appear during the photocatalytic conversion. 
by the work of Abécassis et al. [16,17]. The two methylthioninium chloride solution streams are pumped at $1 \mu \mathrm{L} / \mathrm{min}$ using a Fluigent pressure pump where the flowrate is controlled by individual thermal sensors. The $\mathrm{TiO}_{2}$ suspension containing the same $\mathrm{MB}$ concentration is controlled at the same flowrate using a Nemesys syringe pump. All the solutions have been buffered at $\mathrm{pH} 8.15$ using $1 \mathrm{mM}$ Tris solution. The $365 \mathrm{~nm}$ monochromatic UV light is provided by 2 UV LEDs from Dr. Gröbel that allow a direct control of the intensity without changing the distance. The UV light intensity has been measured at the exact distance by a UVA sensor from the same company.

\subsubsection{Model}

Mass transport is solved numerically for a two-dimensional domain of length $L$ and width $W$. The macroscopic particle distribution was obtained by solving the species conservation equation for both the solute and catalytic particles. While the solute conservation equation includes advection, diffusion, and reaction terms, the particle transport is dictated by advection, migration and diffusion. Note that particle diffusion is insignificant as predicted by the Stokes-Einstein equation. A 3D model has also been built to verify the influence of the channel height. While the particle spreading shows a clear dependency on the $z$ component, there was no specific feature of the integrated $3 \mathrm{D}$ particle profile that was additional to the $2 \mathrm{D}$ model. In dimensionless form the equations are:

$$
\begin{gathered}
\nabla \cdot\left(u c_{1}\right)=\nabla^{2} c_{1}+R \\
\nabla \cdot\left(u c_{2}\right)=D_{2} \nabla^{2} c_{2}+\nabla \cdot\left(\boldsymbol{u}_{D O F} \cdot c_{2}\right)
\end{gathered}
$$

where $c_{i}=\widetilde{c_{i}} / \widetilde{c_{i, 0}}$ is the dimensionless concentration of species $i$ which has diffusivity $D_{i}=\widetilde{D_{i}} / \widetilde{D_{1}}$. Index $i=1$ corresponds to the solute species, while particles are denoted by index $i=2$. $R$ is the normalised pseudo-homogeneous first order reaction rate, $R=D a \cdot c_{1}$, where $D a=k W^{2} / \widetilde{D_{1}}$ is the dimensionless Damköhler number. The apparent reaction rate constant, $k$, scales linearly with the particle density due to the linear proportionality of the catalytic surface area for a monodispersed suspension, $k=k_{0} \cdot c_{2}$, where $k_{0}$ is the intrinsic reaction rate constant. In this study we reference diffusivities to the diffusivity of methylthioninium chloride $\widetilde{D_{1}}=5.7 \times 10^{-10} \mathrm{~m}^{2} / \mathrm{s}$ and concentrations 
a.

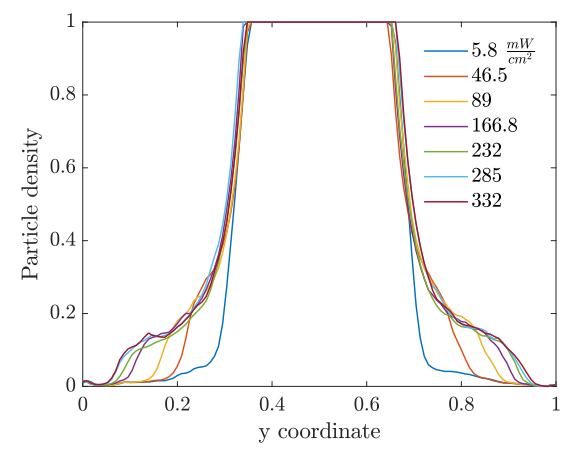

b.

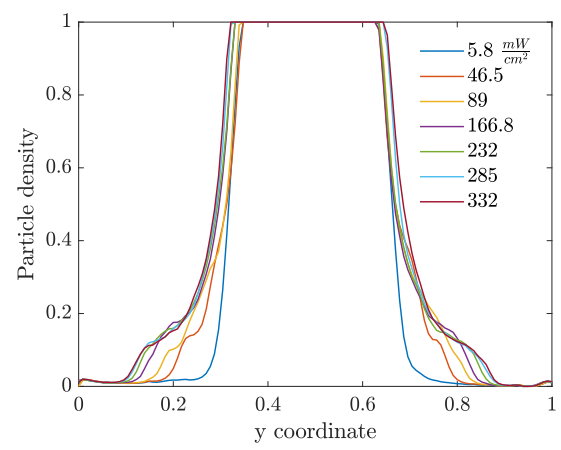

Figure 7.3: 1D particle density profiles for various light intensities and particle concentrations ((a) $1 \mathrm{wt} \% \mathrm{TiO}_{2}$, (b) $0.5 \mathrm{wt} \%, 50 \mu \mathrm{M} \mathrm{MB}, \tau=5.61 \mathrm{~s}$ )

to their corresponding initial values, $\widetilde{c_{i, 0}}$. The probed initial concentrations of methylthioninium chloride in DI water are $\widetilde{c_{1,0}}=10,50$ and $100 \mu \mathrm{M}$, while the initial particles densities are $\widetilde{c_{2,0}}=0.1,0.5$ and $1 \%$. The diffusivity of the particles was estimate using Stokes-Einstein equation, $\widetilde{D_{2}}=4.825 \times 10^{-12} \mathrm{~m}^{2} / \mathrm{s}$. Due to the high aspect ratio, velocity is assumed constant across the width and calculated based on the flowrate and channel dimensions. The $x$ and $y$ coordinates are scaled with respect to the width of the channel $W=600 \times 10^{-6} \mathrm{~m}$. The dimensionless velocity, $u=1595$, is referenced with respect to the diffusion velocity $u=\widetilde{u} W / \widetilde{D_{1}}$. The migration term, $\nabla \cdot\left(\boldsymbol{u}_{D O F} c_{2}\right)$, represents the diffusio-phoresis of particles where the diffusio-phoretic velocity, $\boldsymbol{u}_{D O F}$, scales with the normalized concentration gradient of the solute and has a mobility prefactor that depends on the zeta potential of the particles [186].

$$
\boldsymbol{u}_{D O F}=-\mu_{2} \frac{\nabla c_{1}}{c_{1}}
$$

where the mobility is referenced with respect to the solute diffusivity, $\mu_{2}=$ $\widetilde{\mu_{2}} / \widetilde{D_{1}}$.

$$
\widetilde{\mu_{2}}=-\frac{\varepsilon k_{B} T}{\eta e}\left(\left(\frac{D_{+}-D_{-}}{D_{+}+D_{-}}\right) \zeta-\frac{2 k_{B} T}{e} \ln \left(1-\tanh ^{2}\left(\frac{e \zeta}{4 k_{B} T}\right)\right)\right)
$$

where $\varepsilon$ is the permittivity of water, $k_{B}$ the Boltzmann constant, $T$ the temperature, $\eta$ the viscosity of water, $e$ the elementary charge, $\zeta$ the zeta potential 
and $D_{+}=4.3 \times 10^{-10} \mathrm{~m}^{2} / \mathrm{s}$ and $D_{-}=2.032 \times 10^{-9} \mathrm{~m}^{2} / \mathrm{s}$ are the methylene blue cation and chloride anion diffusivities, respectively [206]. The first term in equation 7.4 represents the electrophoretic contribution coming from the internal electric field set up by the diffusivity contrast of the charged species, i.e. methylene blue cation and chloride anion. The second term encompasses the driving force which originates from the osmotic pressure difference defined in literature as the chemi-phoretic contribution. Both concentration dependent and independent zeta potentials are taken into account.

The three inlets are composed by two different boundary conditions. The side inlets have the reactant concentration 1 and 0 particle density, $c_{1}=1, c_{2}=0$, while the middle inlet has both reactant concentration and particle density 1 , $c_{i}=1$. The outlet boundary condition is open flux, $\frac{\partial c_{i}}{\partial x}=0$. The boundary conditions for the side walls are zero flux, $\frac{\partial c_{i}}{\partial y}=0$.

The governing equations are solved using Finite Element Analysis in COMSOL Multiphysics 5.3. The simulations were performed with a structured mesh, where 500 by 500 rectangular elements were used to ensure mesh independence.

\subsection{Results and discussion}

In the three co-flowing streams the initial methylthioninium chloride (MB) concentration is homogeneous. As long as the particles are passive such that they do not affect the reactant concentration, there is no driving force for diffusio-phoresis. Particle diffusion is negligible in the short 6 seconds residence time, as confirmed in the left panel of figure 7.2. The migration of particles becomes significant when the photocatalytic reaction is triggered by UV light. At this moment the particles start decomposing the methylthioninium chloride, lowering the concentration inside the colloidal stream. The concentration gradient that develops drives the particles towards higher MB concentrations by means of diffusio-phoresis (right figure 7.2). An experiment with the same distribution of particles and a homogeneous $1 \mathrm{mM}$ Tris buffer solution under UV illumination displayed no diffusio-phoresis. We believe this rules out possible heating effects that could arise due to UV light absorption, any gradients generated by radical formation due to solvent (water) reactions that would affect particle migration, any additional gradients arising from Tris buffer oxidation and any $\mathrm{pH}$ gradients. Also, the $\mu \mathrm{M} \mathrm{MB}$ concentration range ensures negligible change in density upon chemical conversion. 

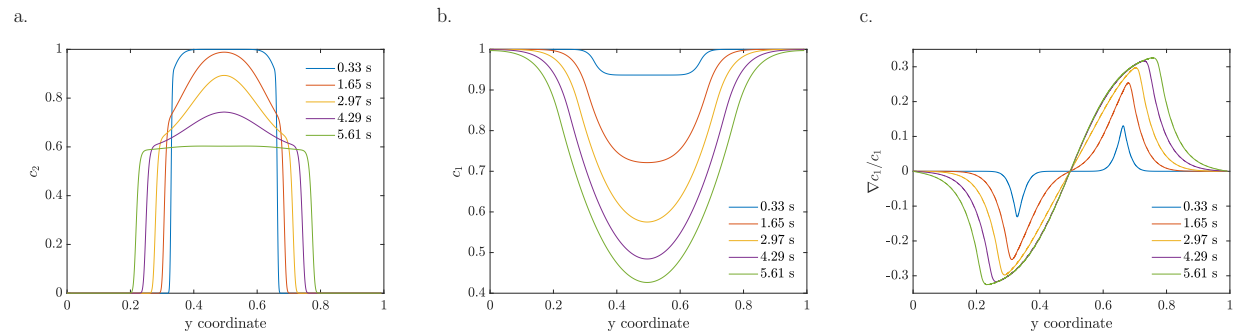

Figure 7.4: Numerically modelled particle (a), reactant (b) concentration profiles and the corresponding reactant normalized gradients $\left(\nabla c_{1} / c_{1}\right)$ (c) for indicated residence times $\left(k=0.2 \mathrm{~s}^{-1}, \zeta=-120 \mathrm{mV}\right)$

The $2 \mathrm{D}$ and the corresponding width averaged $1 \mathrm{D}$ density profiles are shown in Figure 7.2 for different residence times. Upon illumination, a MB gradient appears at the intersection between the streams. This leads to diffusio-phoresis of the catalytic particles from the center stream outwards, where the migrating cloud has a lower particle density compared to the center stream. The local MB conversion depends on the local particle density. As the particles migrate sideways the MB gradient is affected correspondingly. We want to stress the fact that these are plain catalytic particles which are not fluorescent and inherently will give less ideal imaging, including the saturation of the signal in the middle of the particle stream.

The concentration gradients sustained by active particles remain steeper than externally imposed gradients that relax due to diffusion. The particle velocities are extremely high, especially in the beginning when the contrast in particle density is the highest. For example, in the first micrograph slab which has a residence time of only 0.33 seconds, the displacement is already $30 \mu \mathrm{m}$ which corresponds to a diffusio-phoretic velocity of $\sim 90 \mu \mathrm{m} / \mathrm{s}$. The enhancement in migration compared to pure particle diffusion is in the order of $\mathcal{O}\left(10^{3}-10^{4}\right)$. Given that the particle migration is dictated by the local solute gradient, any factor that is altering the concentration profile is affecting the particle distribution. The reaction rate, which is the source for gradient generation, depends on both particle density and light intensity (Figure 7.3). The kinetics for the given particle density and light intensity were estimated from Visan et al. [67] who investigated the same chemistry in an immobilized reactor. It also validates the possibility for neglecting the light intensity decay due to absorption by volume averaging the reaction rate constant, as it falls under the light independence criterion. Assuming that scattering is negligible compared to 

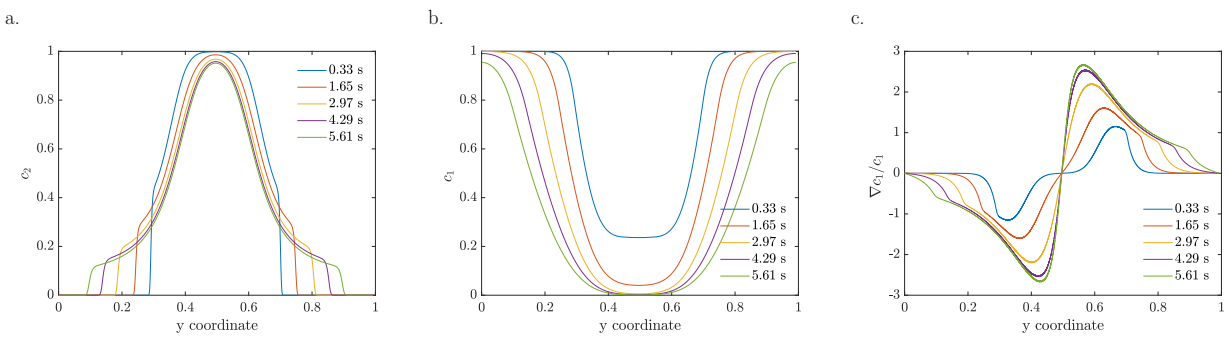

Figure 7.5: Numerically modelled particle (a), reactant (b) concentration profiles and the corresponding reactant normalized gradients $\left(\nabla c_{1} / c_{1}\right)(\mathrm{c})$ for indicated residence times, using a concentration dependent $\zeta$-potential $\left(k=2 \mathrm{~s}^{-1}\right.$, $b=-120 \mathrm{mV}$ )

absorption at the $365 \mathrm{~nm}$ wavelength, this criterion stipulates that the height of the dispersion layer should be smaller than the inverse of the absorption coefficient $\left(\alpha=5.1 \times 10^{3} \mathrm{~m}^{-1}\right)$ for the used $1 \% \mathrm{TiO}_{2}$ particle concentration. That is, for a thickness smaller than $200 \mu \mathrm{m}$, the absorption of light is less than $1-1 / \mathrm{e}(63.2 \%)$ of the incoming light.

We have analyzed the particle distributions using a numerical model that includes the reactant concentration only and its interaction with the catalyst wall, expressed by the mobility (for details, see the methods section). The motivation behind it is that the electro-phoretic contribution is most significant for the starting component as the contrast in diffusivity between the methylthioninium and chloride ions is the highest, i.e. the first term in equation 7.4 dominates. We are aware that the magnitude of the velocity will depend on the collective contribution of all the chemical species present (reactants to intermediates and final products). For the electro-phoretic contribution, as long as the cation diffuses slower than the anion, the resulting electric field will be diminishing the one corresponding to the reactant, but will switch to being cumulative once the cation surpasses the diffusivity of the counter-ion $\left(\mathrm{Cl}^{-}\right.$in this case). For the chemi-phoretic contribution which is dependent on the net interfacial concentration distribution, the product gradient will have an osmotic pressure gradient opposite to the reactant gradient in case of attractive interactions with the catalyst or additional in case of repulsive forces. The complete effect of species gradients on the resulting diffusio-phoretic flow involves many components and their interactions, which is beyond the scope of this paper. Here, we aim to demonstrate the relevance of diffusio-phoresis for heterogeneous catalytic systems. 
The model is based on advection, diffusion and reaction for $\mathrm{MB}$, combined with advection, diffusion and diffusio-phoresis for the catalytic particles. As a starting model, we considered a constant zeta potential, thus constant mobility. The particle density and reactant concentration profiles are shown in Figure 7.4 for $k=0.2 \mathrm{~s}^{-1}$ and $\zeta=-120 \mathrm{mV}$ for the same residence times as the experimental profiles presented in Figure 7.2. For a constant mobility, the particle density evens out as it is spreading. In the beginning, the relative gradient is localised at the edge of the colloidal stream, so only a thin region of particles feel the driving force (e.g. Figure $7.4 \mathrm{c}$ at $0.33 \mathrm{~s}$ ). As the particles spread further, the colloidal stream gets 'diluted' as the maximum relative gradient moves outwards with the edge of the stream and the leading particle density decreases. The reactant gets more depleted in the middle, near $y=0.5$ in Figure $7.4 \mathrm{~b}$, due to higher particle densities, causing these particles to accelerate and catch up with the leading front.

Clearly, the particle distribution does not match the experimental observations, even when the parameters were adjusted. The experimental particle density profiles can be retrieved in the numerical model only when the mobility is decreasing with $\mathrm{MB}$ concentration. The understanding behind a possible decrease in zeta potential with concentration relates to the specifics of the photocatalytic reaction. The degradation of MB follows a complex pathway [215] that produces numerous ionic species which can potentially screen the surface charge. While a clear relationship between the degree of conversion and the zeta potential is difficult to infer a priori, a decrease in zeta potential with reactant concentration can be explained by an increase in the local ionic strength during reaction. Experiments using the same MB conversion, but with additional electrolytes $(10 \mathrm{mM} \mathrm{NaCL})$ confirmed the attenuation of diffusio-phoresis in high ionic strength media. Moreover, the particle dynamics showed experimentally an initial reactant concentration dependency which could not be explained by Langmuir-Hinshelwood kinetics verified numerically using the parameters determined in Houas et al. [215]. This finding suggests a correlation between the particle zeta potential and the reactant concentration for the illuminated state.

We employ a linear function to express the zeta potential in terms of reactant concentration which was also used by Lechnick et al., $\zeta=a+b \cdot c_{1}$. The dependency of the zeta potential on the reactant concentration is an indirect method to account for the change in zeta potential that may be caused by the increase in reaction products. The dependency of the zeta potential on the conversion degree and thus on the reactant concentration is an empirical 
model that attempts to include the (opposing) product contribution on the driving force. A more theoretical model would entail a detailed understanding of the mechanism, that is, not only the identity of these intermediary species, but also their in-situ interaction potential with the catalytic surface and the kinetics for all elementary reactions, i.e. the change in the distribution of these species with the conversion degree.

The particle density and reactant concentration profiles shown in Figure 7.5 for $k=2 \mathrm{~s}^{-1}$ and $a=0 \mathrm{mV}, b=-120 \mathrm{mV}$ match the experimental shapes presented in Figure 7.2. The fitted starting value $(-120 \mathrm{mV})$ is relatively close to what has been reported in literature [208]. Leroy et al. found a zeta potential of $-90 \mathrm{mV}$ for the same Evonik $\mathrm{TiO}_{2}$ particles at $\mathrm{pH} 8.15$ corresponding to the conditions used in our experiments. The difference could be explained by an in-situ increase in zeta potential upon UV light illumination which may originate from the same mechanism by which photocatalysis takes place, i.e. the generation of positive and negative charges that migrate to the surface. The concentration dependent zeta potential has the same initial magnitude, but decays very fast as the reactant is consumed which leads to a decreased mobility. It is very clear from Figure $7.5 \mathrm{c}$ that the maximum in relative gradient does not move outwards with the edge of the particle stream, but instead remains deeper inside the particle stream. Here, the absolute reactant concentration is quite low, leading to a very strong relative gradient. However, due to the low reactant concentration in this middle region, the mobility is also very low. The consequence is not only a significantly reduced overall migration, but also a reduced particle density at the migrating front. Particles are continuously left behind as the spreading develops due to the steep decrease in reactant concentration in the migrating front. This gradual decline in the particle front density also reduces the driving force by relaxing the concentration gradient in Figure 7.5c.

The particle dynamics are very sensitive to the interplay between the activity of the particles and their surface properties via the mobility. The mobility represents the sensitivity of the particle's response to the gradient (Figure 7.6a). Enhanced zeta potentials are directly reflected in enhanced migration. For higher catalytic activities, steeper gradients develop which drive higher diffusio-phoretic velocities. Because the mobility depends on the local concentration, a change in the reaction rate constant will affect both the relative gradient as well as the mobility prefactor via the zeta potential which will couple back to the concentration profile in a nonlinear way (Figure 7.6b).

The spontaneous response of catalytic particles to the local reactant gradient 
a.

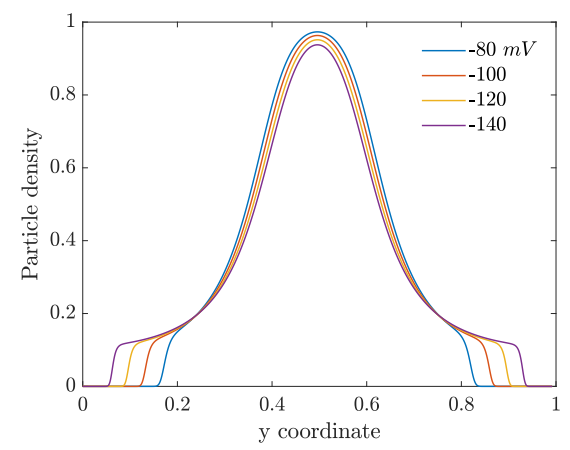

b.

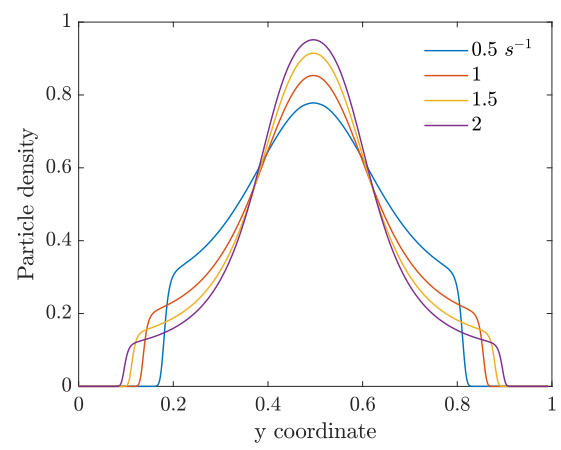

Figure 7.6: Particle density profiles at $\tau=5.61 \mathrm{~s}$ for a. various concentration dependent $\zeta\left(k=2 \mathrm{~s}^{-1}\right)$ and $\mathrm{b}$. various $k(b=-120 \mathrm{mV})$

enhances overall conversion. As they migrate towards regions of higher concentration, the local reaction rate increases. The conversion enhancement will depend on the extent of the particle migration, being a function of catalyst activity, its surface properties and residence time. As an example, given the parameters used in Figure 7.5, the conversion doubles for the final residence time of 5.6 seconds with respect to a migration free scenario.

\subsection{Conclusions}

This work illustrates some intriguing aspects for reaction induced diffusiophoresis of plain catalytic particles. While photocatalytic degradation of methylthioninium chloride may not pass as a standard industrial catalytic conversion, we consider the photocatalytic example to be an experimental proof for a general transport mechanism by which active particles migrate in response to local gradients that are produced by the particles themselves through their uneven distribution. We believe this phenomenon to be present for arbitrary chemical conversions whenever their kinetics are fast enough to preserve significant concentration gradients. While the phenomenon is general, we expect the particle migration signature to be specific to the catalytic system, depending on kinetics and interaction of all species involved. A simple numerical model is used to explore the details of the diffusio-phoretically driven process, confirming the relevance of the molecular interactions between 
the reactants/products and the catalyst surface. 


\section{Summary and Outlook}

\subsection{Summary}

As presented in the last century in chemical engineering textbooks $[90,91$, 95], there has been a general consent on the diffusion based mass transport inside porous catalysts. As pressure driven flows are forbidden at this small dimensions, convective transport seems to be out of the question. The famous Ernest Thiele pointed out that pellets of excellent catalyst are active only at the surface, [184] given the underlying assumption that diffusion is the only replenishment mechanism inside the catalytic pores.

In this thesis we challenge the generally accepted narrative on mass transport for heterogeneous catalysis and demonstrate that convective transport inside catalytic dead-end pores is not only possible, but ubiquitous for higher kinetics. Surface flows induced by concentration gradients, i.e. diffusio-osmotic flows, form during catalysis inside the pores. This additional mass transport does not require any external input. It spontaneously arises when the configuration of the catalyst matrix facilitates the development of significant gradients with respect to the bulk solution. The reaction driven surface flow which originates in the osmotic pressure gradient and diffusion potential in case of charged species, replenishes the catalytic pores with fresh solution, having a positive impact on the conversion. We visualise and quantify the flow in 3D using the General Defocusing Particle Tracking technique. We analyse the phenomena using a model that includes the fluid dynamics actuated by the concentration gradients that arise due to the catalytic reaction. We are able to extract parameters revealing the interaction strength between the reactant/product 
chemical species and the catalytic surface.

Diffusion is also the dominating mass transport mechanism close to the catalyst surface where external mixing loses its efficiency. We introduce the concept of enhancing external transport by patterning catalysts. The reactivity contrast designed by alternating active and inactive regions generates spontaneously in-plane gradients that drive a steady diffusio-osmotic flow. While the details of the numerical model may need to be adjusted for particular catalytic reactions, the approach is not pinned to a certain chemistry. Concentration gradients will develop as long as kinetics are fast enough to place the system in the mass transfer limiting regime. The diffusio-osmotic flow is redirected out of plane due to the symmetry of the system and mixes the otherwise diffusion dominated boundary layer which greatly enhances mass transport and thus impacts the overall conversion. Scaling laws provide a direct correlation between the catalytic chemistry, the dynamics of the system, and the conversion enhancement. Specific chemistries, with known reaction kinetics and interaction potentials, can now be tested against this predictive model.

Slurry reactors are known for their high conversion capacity as they benefit from high mass transfer rates. This has been explained so far by accessible high catalytic surface areas and small diffusion length scales. In this thesis we demonstrate experimentally an overlooked transport mechanism by which particles migrate towards higher reactant concentration. Whenever an uneven particle distribution appears, the higher particle density region will lower the reactant concentration more than the surrounding environment. This macroscopic concentration gradient propels the particles towards higher reactant concentration by diffusio-osmotic flows. We believe this phenomenon to be present for arbitrary chemical conversions whenever their kinetics are fast enough to preserve significant concentration gradients. We have analysed the experimental particle distribution by means of a numerical model that includes diffusio-phoretic movement of the catalytic particles. While the phenomenon is general, the particle migration signature is specific to the catalytic system, depending on kinetics and molecular interactions between the reactant/product species and the catalyst surface.

\subsection{Outlook}

While this thesis is a proof of principle for using in-situ reaction driven diffusioosmotic flows to enhance mass transport in ordinary catalytic systems, there 
are multiple aspects that need to be investigated further. The fundamental theory regarding diffusio-osmotic phenomena for reactive systems needs to be validated and developed further. Sampling multiple chemistries is a prerequisite for formulating general principles.

Chemical reactions involve multiple species, from reactants to intermediates and products. All these elementary steps as well as their individual kinetics need to be known to have an accurate description of the transient concentration profiles. To be able to quantify the corresponding individual driving forces, their intrinsic mobilities are also required. These mobilities are a measure of the integrated concentration across the interaction length which is dictated by the individual interaction potential. One important observation here is that in Chapter 5, we experimentally determined mobilities much higher than the values approximated for inert surfaces. A possibility would be an in-situ increase in the interaction potential. This alteration as a condition for reaction is already speculated for photocatalysts and for high temperature metal catalysts. In the first example the surface charge increases upon UV-Vis illumination by the charge carrier generation mechanism particular for semiconductors. In the second example metal surface morphology changes upon heating which enhances the coordination degree with the chemical species. That is why well known chemistries need to be employed to validate the additional degree of complexity that reactive systems pose.

The next question arises about the summation of these individual driving forces to calculate the net chemi-osmotic and electro-osmotic contributions. There is no guarantee that individual contributions add up linearly and most likely they will have to be weighted against the relative composition of these individual species inside the interaction potential length scale. The implications will be transient net mobilities that depend on the conversion degree. A preliminary proof for the relevance of the composition of the interaction layer is the quenching of the diffusio-osmotic phenomenon at high ionic strength. If the charged surface is screened completely by an inert salt, the discreet presence of other species will not make up for an overall osmotic pressure gradient despite their individual concentration gradient. The internal electric field due to diffusivity contrast will also most likely be screened by the high permittivity medium.

Another uncertainty that naturally emerges is the lower limit of the absolute concentration of the chemical species for which the diffusio-osmotic fundamental theory holds. This is especially relevant for catalytically induced flows. While computationally we set a random limit of $10^{-9} \mathrm{M}$, experiments are re- 


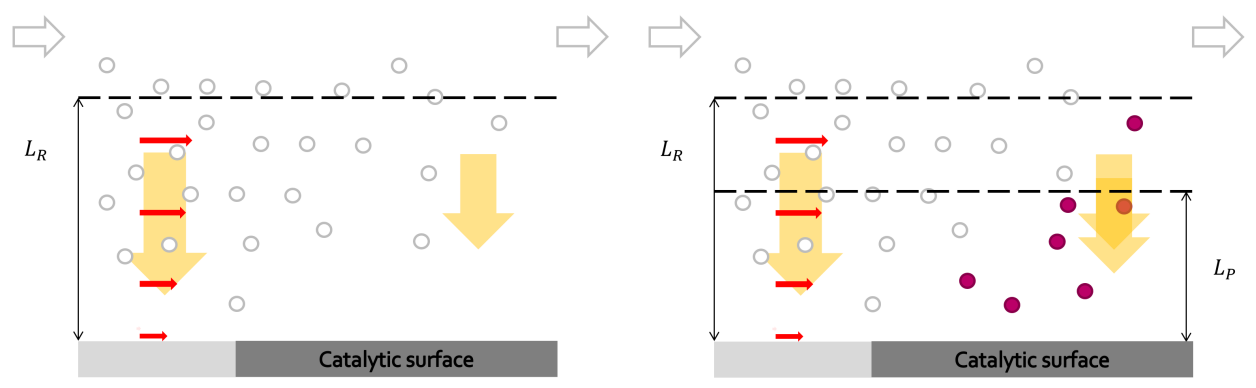

Figure 8.1: Illustration for in-situ driven diffusio-osmotic flow by active surfaces. The left side accounts only for reactant consumption. The right side includes the possible opposing driving force given by a mirrored product concentration profile.

quired to identify where the continuity assumption breaks down. Regarding the upper limit, it is expected that for high absolute concentration values the bulk gradient will not translate anymore into a gradient inside the interaction potential layer, as the screening of the surface is saturated above certain molecular densities. Although for highly active catalysts that consume/convert the species present at their surface this condition may be overcome as the equilibrium composition is continuously altered leaving room for out of equilibrium dynamics. Especially for in-situ generated concentration gradients that appear as a result of the surface flow itself, this condition could be realistically met.

Regarding the diffusio-osmotic flow inside catalytic dead-end pores, an important question arises about the prospect of this surface flow in increasingly narrower pores. One concern regards the overlap of these interaction potential layers that would prohibit an opposing pressure driven flow. The second concern is the high pressure drop at small diameters that will counteract the inward surface driven flow. If these limitations do prove to be true they could still be overcome by $3 \mathrm{D}$ patterning in the nanometer range to allow for the assembly of symbiotic materials that combine the inward surface flow with an outward surface flow. This fortunate situation was also present in Chapter $\mathbf{5}$ where the PDMS top assisted the recirculation by an outward surface driven flow. 

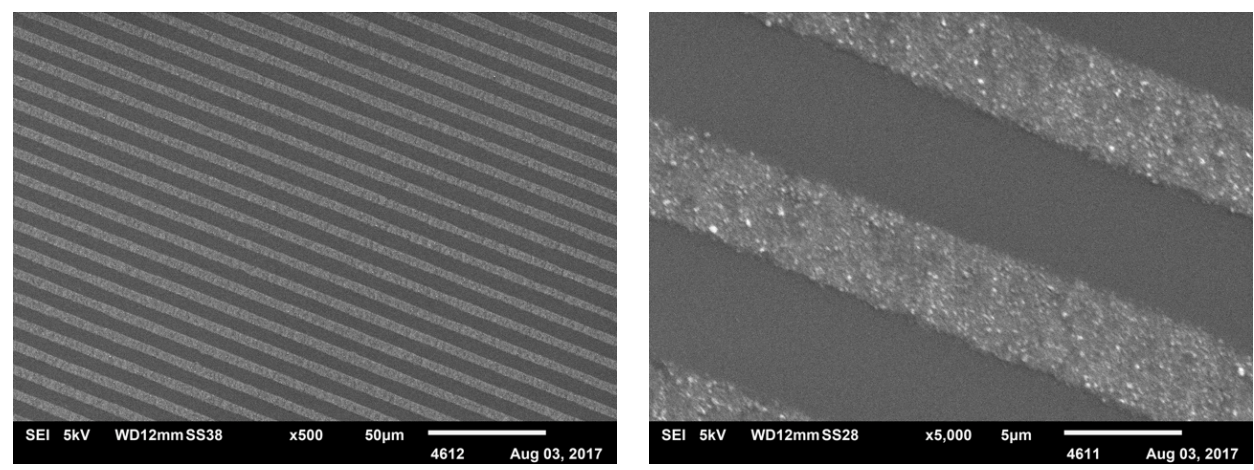

Figure 8.2: High resolution SEM of patterned porous catalyst $\left(\mathrm{TiO}_{2}\right)$.

\section{Research infrastructure}

All these propositions stemming from the high degree of complexity for reactive systems need to be settled experimentally and complemented by molecular dynamics simulations. The only approach at hand is to design well-defined protocols that are based on complete chemical information regarding (in-situ) interaction potentials and kinetics of elementary reactions. This experimental endeavour requires extensive preliminary investigation. To identify all possible intermediates involved in the mechanistic pathway and resolve their individual kinetics, online mass spectrometry with time resolution in the milliseconds is required. This has already been reported for $\mu$ total analysis systems [216,217]. To be able to sample online the surface composition ATR-IR spectrometry proved to be a useful technique [218, 219].

The microfluidic platform that is undoubtedly required for the times scales and length scales relevant for these investigations poses another technical challenge concerning the patterning of porous catalyst inside microchannels. The local deposition of materials using methods compatible with photolithography is the easy task. However, these cleanroom techniques such as sputtering and vapour deposition lead to dense materials with low surface area and low catalytic activity. Among the promising techniques are flame spray deposition, glancing angle vapour deposition, and electrical discharge [220-222]. These deposition methods required designated infrastructure and extensive optimization. We selected for research purposes the local dip coating via contrast in wettability. This entails the chemical modification of the support using silanes which converts the initial hydrophilic surface into a hydrophobic one. This surface functionalization can be patterned by photolithography techniques. 
After hydrophobization, the photoresist is stripped to form hydrophobic and hydrophilic patterns. When contacting the heterogeneous surface with the catalyst suspension, it adheres preferentially only on the hydrophilic patches, being repelled by the hydrophobic ones. The resolution of this technique goes down to $5 \mu \mathrm{m}$ (Figure 8.2).

\section{Diffusio-osmosis as a tool for fundamental studies}

If these numerous complexities that arise for reactive surfaces are resolved and the additional theory is systematically confirmed, we can envision using this phenomena to develop a new in-situ characterisation method for investigating the interaction between the chemical species and the catalyst surface. We have conducted the analysis in Chapter $\mathbf{6}$ by assuming an independence between the reactivity of the surface and diffusio-osmotic mobility. It is very likely that these properties are intimately connected as the interaction forces of the numerous species with the surface is what determines the reactivity. The device designed by Shin and coworkers [189] is an inspirational example in that aspect. Of course, reactive systems are in a league of their own and require more efforts regarding infrastructure and operation. Another inspirational work comes from Palacci et al. $[28,29]$ that hints at the level of complexity required to achieved coordinated response. This type of approach could be transferred from precise manipulation to analysing patterns emerging from out of equilibrium conditions. Collecting the signature from a series of events such as the intersection of pairs of streams of different residence times or different particle densities could deconvolute the contribution of the different chemical species to the net driving force.

\section{Perspectives on industrial design}

The relevance of catalytically driven diffusio-osmosis may go beyond enhanced mass transport and fundamental studies. There are foreseen implications for the local residence time of products close to the catalyst surface which could prevent secondary reactions that would decrease the selectivity and potentially poison the catalyst. The research question here is can the design of the catalyst, beyond material development, through mere geometric arrangement enhance the yield and selectivity of the process and even prevent deactivation of the catalyst? Designs where particles are guided continuously by the fresh supply of reactants to prevent consecutive reactions may actually prevent deactivation of the catalyst. Using the same strategy may even assist or bypass 
the final particle separation as streams with the aimed degree of conversion could be withdrawn as the particles move to higher concentration sites. This is achievable as diffusio-phoresis precedes solute diffusion. This added value comes at a very low price as the distribution of particles is the only design criterion. There is no requirement regarding complex particle synthesis or use of additional chemicals.

The concept of surface heterogeneity started from the intuitive idea that consecutive reactions would benefit from alternating catalysts in order to consume intermediates that would shift the thermodynamic equilibrium of the reaction and increase overall conversion. The obvious concentration gradients that would arise inspired the investigation of the more subtle phenomenon of catalytically induced diffusio-osmosis. Due to the higher complexity of a chemical system compromising consecutive reactions, the phenomenon was explored by reducing the contrast in activity to alternating active and inactive patches. It goes without saying that complementary catalysts that would produce and consume species adjacently would lead to steeper gradients. This is a strong incentive for not abandoning the initial idea. 


\section{Samenvatting}

Zoals in de afgelopen eeuw, gepresenteerd in chemische technologie boeken $[90,91,95]$, is er een algemene consensus over het op diffusie gebaseerd transport in poreuze katalysatoren. Omdat het op deze kleine schaal niet mogelijk is om door druk aangedreven stromen te laten plaatsvinden, is er geen transport door middel van convectie mogelijk. De beroemde Ernest Thiele toonde aan dat diffusie het enige mechanisme is, in katalytische poriën van kwalitatief hoogwaardige pellets welke enkel aan het oppervlak actief zijn [184].

In dit proefschrift testen we het algemeen geaccepteerde verhaal aangaande massatransport voor heterogene katalysatoren en laten we zien, dat convectie transport in katalytische doodlopende poriën niet alleen mogelijk is, maar ook zorgt voor snellere reacties. Oppervlaktestromen, opgewekt door een concentratiegradiënt zoals bijvoorbeeld diffusie-osmotische stromen, vormen zich gedurende de katalytische reacties in de poriën. Dit additionele massatransport vereist geen externe bron. Het ontstaat spontaan wanneer de structuur van de katalysatormatrix de ontwikkeling van significante concentratiegradiënten, ten opzichte van de bulkoplossing, stimuleert. De door de reactie aangedreven oppervlakte stroom die zijn oorsprong vindt in de osmotische drukgradiënt en de diffusiepotentiaal, als er sprake is van geladen deeltjes, voorziet de katalytische poriën van verse oplossing. Dit resulteert in een hogere conversie. We visualiseren en kwantificeren de stroom in 3D met behulp van de General Defocusing Paticle Tracking techniek. We analyseren de fenomenen met behulp van een vloeistof dynamisch model, aangedreven door de concentratie gradiënten die ontstaan door de katalytische reactie. We zijn in staat om hier parameters uit te extraheren die de interactie sterkte tussen de reactant/chemisch product en het katalytische oppervlak aangeven. Diffusie is ook het dominante massatransport mechanisme in de nabijheid van het katalysator oppervlakte omdat het externe mixen zijn efficiëntie verliest nabij het oppervlakte. Wij introduceren het concept van verhoogd extern transport door patronen te maken op de katalysatoren. Het reactiviteitscon- 
trast, gevormd door actieve en inactieve regionen zorgen voor spontante gradiënten, parallel aan het oppervlak, welke zorgen voor een diffusie-osmotische stroom. Hoewel de details van het numerieke model aangepast dienen te worden voor specifieke katalytische reacties, is de aanpak niet uitsluitend voor een specifieke chemische reactie. Concentratiegradiënten zullen ontstaan wanneer de uitwisseling snel genoeg is om het systeem zich bevindt in het massaoverdracht limiterende regime. De diffusie-osmotische stroom is, door de symmetrie van het systeem, uit het oppervlakte en zorgen voor het mengen in het anders door diffusie gedomineerde grensvlak. Dit verhoogt de hoeveelheid massatransport en heeft daardoor een positieve invloed op de algehele conversie. Schalingswetten geven een directie correlatie tussen de katalytische chemie, de dynamica van het systeem en verhoging van de conversie. Specifieke chemische reacties met bekende reactiesnelheden en interactie potentialen kunnen tegen dit voorspellende model gehouden worden.

Slurryreactoren staan bekend om hun hoge conversie capaciteit door hoge massatransport snelheden. Tot op heden wordt dit gewijd aan de toegankelijke hoog katalytische oppervlakten en korte diffusielengtes. In dit proefschrift demonstreren we experimenteel een over het hoofd gezien transport mechanisme, waarbij katalytische deeltjes migreren naar een hogere reactant concentratie. Wanneer er sprake is van een ongelijke verdeling van de katalytische deeltjes, zullen de regionen met een hogere dichtheid van deeltjes de reactant concentratie meer verlagen dan de omliggende regionen. Deze macroscopische concentratie gradiënt drijven de deeltjes naar de hoge reactant concentratie regionen door diffusie-osmotische stromen. We geloven dat dit fenomeen aanwezig is bij verscheidene chemische conversies zolang de reacties maar snel genoeg zijn om de significante concentratie gradiënten in stand te houden. We hebben de experimentele verdeling van deeltjes geanalyseerd door een numeriek model dat rekening houdt met de diffusiephoretische beweging van katalytische deeltjes. Hoewel het fenomeen veralgemeniseerbaar is, is de migratie van deeltjes specifiek voor het katalytische systeem. Welke afhankelijk is van de reacties die plaatsvinden en de moleculaire interacties tussen de reactant/het product en het katalysator oppervlak. 


\section{Acknowledgements}

If you think about it, all wealth has been produced collectively - through recycling and through a gradual accumulation of knowledge. Workers need entrepreneurs to hire them, who need workers to buy their goods. Entrepreneurs need bankers to lend to them, who need entrepreneurs to pay interest. Bankers need governments to protect them, who need bankers to fuel the economy. Inventors cannibalize the inventions of others and plagiarize the ideas of scientists. The economy relies on everybody. - Yanis Varoufakis

Thank you Rob for giving me the opportunity to do a PhD in SFI and thank you for taking me back. I learned so much from you on all aspects and I hope you know I appreciated every single time you made time to help me. I am grateful for your kindness, generosity, patience, peacefulness and the so many other ways that make you such an amazing person. I will miss you a great deal.

Thank you Jeff for sharing your vast knowledge and for always being willing to lend a hand. Thank you Alvaro for teaching me the GDPT technique, for your beautiful plots and for your encouragement. Nothing went unnoticed. Thank you Jan for the numerous times you helped me in the lab. I know it was the most you could do in your overloaded schedule. Thank you Sip Jan for helping me fabricate the many different devices and for always being so approachable and friendly. Thank all the cleanroom staff and users who lent me a hand in my lonely struggle in the cleanroom. Thank you Kees for making time to help me even if I know you never had the time. I hope you can give me a ride to DTU someday. They have the large wafers, you know?! Thank you Christina, Eiko, Hai, Khalid, Vasilis for helping me when nobody else did. It is greatly appreciated. Thank you Lidy, Brigitte, Audrey and so many other colleagues that were kind and friendly. I wish I had more time to join the coffee breaks and enjoy your company.

I would also like to extend my gratitude to all the committee members that took time off their busy agendas to read this thesis and participate in the 
defence.

I had the fortune to meet some awesome people throughout these years that made my experience in Enschede so great. I have so many wonderful memories with all of you. There is just too much to say. In alphabetical order, Agi, Akansha, Albert, Alberto, Alvaro, Ameya, Beata, Can, Carola, Christina, Corentin, Damon, Denys, Dorota, Douwe, Eiko, Elena, Enzo, Erik, Fokje, Ginteras, Hai, Hani, Hoa, Ivan, JB, Jacques, Jasper, Jeroen, José, Josh, Juan, Khalid, Krzysztof, Lionel, Mandakranta, Maria, Marina, Marzena, Mazi, Miguel, Nicole, Özlem, Pelin, Pim, Renaud, Renée, Rodrigo, Roger, Taylan, Timon, Valentina, Vasilis and so many others.

Thank you Andrei for all your support. Multumesc parintilor mei, Maria si Viorel, pentru ca m-au incurajat mereu sa fac ce imi doresc si au muncit incredibil de mult sa se asigure ca se poate indeplini.

With love,

Aura

May 2019 


\section{References}

[1] Yanbing Guo, Gang Liu, Zheng Ren, Adimali Piyadasa, and Pu-Xian Gao. Single crystalline brookite titanium dioxide nanorod arrays rooted on ceramic monoliths: a hybrid nanocatalyst support with ultra-high surface area and thermal stability. CrystEngComm, 15(41):8345, 2013.

[2] B V Derjaguin, S S Dukhin, and M M Koptelova. Capillary osmosis through porous partitions and properties of boundary layers of solutions. Journal of Colloid And Interface Science, 38(3):584-595, 1972.

[3] B.V. Derjaguin, G Sidorenkov, E Zubashchenko, and E Kiseleva. Kinetic Phenomena in the boundary layers of liquids 1 . the capillary osmosis, 1993.

[4] B.V. Derjaguin, S.S. Dukhin, and A.A. Korotkova. Diffusiophoresis in electrolyte solutions and its role in the Mechanism of the formation of films from caoutchouc latexes by the ionic deposition method. Progress in Surface Science, 43(1-4):153-158, may 1993.

[5] J. L. Anderson, M. E. Lowell, and D. C. Prieve. Motion of a particle generated by chemical gradients Part 1. Non-electrolytes. Journal of Fluid Mechanics, 117(-1):107, apr 1982.

[6] D C Prieve, J L Anderson, J P Ebel, and M E Lowell. Motion of a particle generated by chemical gradients. Part 2. Electrolytes. Journal of Fluid Mechanics, 148(-1):247, nov 1984.

[7] John L. Anderson and Dennis C. Prieve. Diffusiophoresis Caused by Gradients of Strongly Adsorbing Solutes. Langmuir, 7(2):403-406, 1991.

[8] John L. Anderson. Effect of nonuniform zeta potential on particle movement in electric fields. Journal of Colloid and Interface Science, 105(1):45-54, may 1985. 
[9] M C Fair and J L Anderson. Electrophoresis of nonuniformly charged ellipsoidal particles. Journal of Colloid And Interface Science, 127(2):388400, 1989.

[10] Ronald E. Smith and Dennis C. Prieve. Accelerated deposition of latex particles onto a rapidly dissolving steel surface. Chemical Engineering Science, 37(8):1213-1223, 1982.

[11] Mitchell M J Lin and Dennis C Prieve. Electromigration of latex induced by a salt gradient. Journal of Colloid And Interface Science, 95(2):327339, 1983.

[12] W. Jay Lechnick and Joseph A. Shaeiwitz. Measurement of diffusiophoresis in liquids. Journal of Colloid And Interface Science, 102(1):7187, nov 1984.

[13] W Jay Lechnick and Joseph A Shaeiwitz. Electrolyte concentration dependence of diffusiophoresis in liquids. Journal of Colloid And Interface Science, 104(2):456-470, 1985.

[14] Sangwoo Shin, Eujin Um, Benedikt Sabass, Jesse T Ault, Mohammad Rahimi, Patrick B Warren, and Howard A Stone. Size-dependent control of colloid transport via solute gradients in dead-end channels. Proceedings of the National Academy of Sciences, 113(2):257-261, 2016.

[15] Peter O. Staffeld and John A. Quinn. Diffusion-induced banding of colloid particles via diffusiophoresis 1. Electrolytes. Journal of Colloid and Interface Science, 130(1):69-87, jun 1989.

[16] B. Abécassis, C. Cottin-Bizonne, C. Ybert, A. Ajdari, and L. Bocquet. Osmotic manipulation of particles for microfluidic applications. New Journal of Physics, 11(7):075022, jul 2009.

[17] B. Abécassis, C. Cottin-Bizonne, C. Ybert, A. Ajdari, and L. Bocquet. Boosting migration of large particles by solute contrasts. Nature Materials, 7(10):785-789, oct 2008.

[18] Peter O. Staffeld and John A. Quinn. Diffusion-induced banding of colloid particles via diffusiophoresis 2. Non-Electrolytes. Journal of Colloid and Interface Science, 130(1):88-100, jun 1989.

[19] Marek Kosmulski and Egon Matuevi. Solvophoresis of latex. Journal of Colloid And Interface Science, 150(1):291-294, 1992. 
[20] Joel S. Paustian, Craig D. Angulo, Rodrigo Nery-Azevedo, Nan Shi, Amr I. Abdel-Fattah, and Todd M. Squires. Direct measurements of colloidal solvophoresis under imposed solvent and solute gradients. Langmuir, 31(15):4402-4410, 2015.

[21] Rodrigo Nery-Azevedo, Anirudha Banerjee, and Todd M. Squires. Diffusiophoresis in Ionic Surfactant Gradients. Langmuir, 33(38):9694-9702, 2017.

[22] Nan Shi, Rodrigo Nery-Azevedo, Amr I Abdel-Fattah, and Todd M Squires. Diffusiophoretic Focusing of Suspended Colloids. Physical Review Letters, 117(25):1-5, 2016.

[23] Rajarshi Guha, Xia Shang, Andrew L Zydney, Darrell Velegol, and Manish Kumar. Diffusiophoresis contributes significantly to colloidal fouling in low salinity reverse osmosis systems. Journal of Membrane Science, 479:67-76, 2015.

[24] Abhishek Kar, Rajarshi Guha, Nishant Dani, Darrell Velegol, and Manish Kumar. Particle deposition on microporous membranes can be enhanced or reduced by salt gradients. Langmuir, 30(3):793-799, 2014.

[25] Joseph J. McDermott, Abhishek Kar, Majd Daher, Steve Klara, Gary Wang, Ayusman Sen, and Darrell Velegol. Self-generated diffusioosmotic flows from calcium carbonate micropumps. Langmuir, 28(44):1549115497, 2012.

[26] Abhishek Kar, Tso Yi Chiang, Isamar Ortiz Rivera, Ayusman Sen, and Darrell Velegol. Enhanced transport into and out of dead-end pores. ACS Nano, 9(1):746-753, 2015.

[27] Jesse T. Ault, Patrick B. Warren, Sangwoo Shin, and Howard A. Stone. Diffusiophoresis in one-dimensional solute gradients. Soft Matter, 13(47):9015-9023, 2017.

[28] Jérémie Palacci, Benjamin Abécassis, Cécile Cottin-Bizonne, Christophe Ybert, and Lydéric Bocquet. Colloidal motility and pattern formation under rectified diffusiophoresis. Physical Review Letters, 104(13):1-4, 2010 .

[29] Jérémie Palacci, Cécile Cottin-Bizonne, Christophe Ybert, and Lydéric Bocquet. Osmotic traps for colloids and macromolecules based on logarithmic sensing in salt taxis. Soft Matter, 8(4):980-994, 2012. 
[30] Alessandro Siria, Philippe Poncharal, Anne Laure Biance, Rémy Fulcrand, Xavier Blase, Stephen T. Purcell, and Lydéric Bocquet. Giant osmotic energy conversion measured in a single transmembrane boron nitride nanotube. Nature, 494(7438):455-458, 2013.

[31] Hiroaki Yoshida, Sophie Marbach, and Lydéric Bocquet. Osmotic and diffusio-osmotic flow generation at high solute concentration. II. Molecular dynamics simulations. The Journal of Chemical Physics, 146(19):194702, may 2017.

[32] Ran Niu, Patrick Kreissl, Aidan T Brown, Georg Rempfer, Denis Botin, Christian Holm, Thomas Palberg, and Joost De Graaf. Microfluidic pumping by micromolar salt concentrations. Soft Matter, 13(7):1505$1518,2017$.

[33] Yiying Hong, Misael Diaz, Ubaldo M. Córdova-Fteueroa, and Ayusman Sen. Light-driven titanium-dioxide-based reversible microfireworks and micromotor/micropump systems. Advanced Functional Materials, 20(10):1568-1576, 2010.

[34] Walter F. Paxton, Paul T. Baker, Timothy R. Kline, Yang Wang, Thomas E. Mallouk, and Ayusman Sen. Catalytically induced electrokinetics for motors and micropumps. Journal of the American Chemical Society, 128(46):14881-14888, nov 2006.

[35] Yang Wang, Rose M. Hernandez, David J. Bartlett, Julia M. Bingham, Timothy R. Kline, Ayusman Sen, and Thomas E. Mallouk. Bipolar electrochemical mechanism for the propulsion of catalytic nanomotors in hydrogen peroxide solutions. Langmuir, 22(25):10451-10456, 2006.

[36] S. Ebbens, D. A. Gregory, G. Dunderdale, J. R. Howse, Y. Ibrahim, T. B. Liverpool, and R. Golestanian. Electrokinetic effects in catalytic platinum-insulator Janus swimmers. Epl, 106(5):1-5, jun 2014.

[37] Jeffrey L. Moran and Jonathan D. Posner. Electrokinetic locomotion due to reaction-induced charge auto-electrophoresis. Journal of Fluid Mechanics, 680:31-66, 2011.

[38] R. Golestanian, T. B. Liverpool, and A. Ajdari. Designing phoretic micro- and nano-swimmers. New Journal of Physics, 9, 2007. 
[39] Ramin Golestanian, Tanniemola B. Liverpool, and Armand Ajdari. Propulsion of a Molecular Machine by Asymmetric Distribution of Reaction Products. Physical Review Letters, 94(22):220801, jun 2005.

[40] Vinita Yadav, Hua Zhang, Ryan Pavlick, and Ayusman Sen. Triggered "on/off" micropumps and colloidal photodiode. Journal of the American Chemical Society, 134(38):15688-15691, 2012.

[41] Hua Zhang, Kimy Yeung, Jessica S Robbins, Ryan A Pavlick, Meng Wu, Ran Liu, Ayusman Sen, and Scott T Phillips. Self-powered microscale pumps based on analyte-initiated depolymerization reactions. Angewandte Chemie - International Edition, 51(10):2400-2404, 2012.

[42] Chao Zhou, Hua Zhang, Zeheng Li, and Wei Wang. Chemistry pumps: A review of chemically powered micropumps. Lab on a Chip, 16(10):17971811, 2016.

[43] Samuel Sanchez, Lluis Soler, and Jaideep Katuri. Chemically powered micro- and nanomotors. Angewandte Chemie - International Edition, 54(5):1414-1444, 2015.

[44] Darrell Velegol, Astha Garg, Rajarshi Guha, Abhishek Kar, and Manish Kumar. Origins of concentration gradients for diffusiophoresis. Soft Matter, 12(21):4686-4703, 2016.

[45] Armand Ajdari and Lydéric Bocquet. Giant amplification of interfacially driven transport by hydrodynamic slip: Diffusio-osmosis and beyond. Physical Review Letters, 96(18):1-4, 2006.

[46] Sébastien Michelin, Thomas D. Montenegro-Johnson, Gabriele De Canio, Nicolas Lobato-Dauzier, and Eric Lauga. Geometric pumping in autophoretic channels. Soft Matter, 11(29):5804-5811, 2015.

[47] Huan J. Keh. Diffusiophoresis of charged particles and diffusioosmosis of electrolyte solutions. Current Opinion in Colloid and Interface Science, 24:13-22, 2016.

[48] Tom Van Gerven, Guido Mul, Jacob Moulijn, and Andrzej Stankiewicz. A review of intensification of photocatalytic processes. Chemical Engineering and Processing: Process Intensification, 46(9):781-789, sep 2007. 
[49] Camilo A. Arancibia-Bulnes, Antonio E. Jiménez, and Claudio A. Estrada. Development and Modeling of Solar Photocatalytic Reactors. In Advances in Chemical Engineering, volume 36, pages 185-227. 2009.

[50] Meng Ni, Michael K.H. Leung, Dennis Y.C. Leung, and K. Sumathy. A review and recent developments in photocatalytic water-splitting using $\mathrm{TiO} 2$ for hydrogen production. Renewable and Sustainable Energy Reviews, 11(3):401-425, apr 2007.

[51] Sai Wei Lam, Ken Chiang, Tuti M. Lim, Rose Amal, and Gary K.C. Low. The effect of platinum and silver deposits in the photocatalytic oxidation of resorcinol. Applied Catalysis B: Environmental, 72(3-4):363-372, mar 2007.

[52] J. Ruud van Ommen, Dirkjan Kooijman, Mark de Niet, Mojgan Talebi, and Aristeidis Goulas. Continuous production of nanostructured particles using spatial atomic layer deposition. Journal of Vacuum Science $\mathbb{E}$ Technology A: Vacuum, Surfaces, and Films, 33(2):021513, mar 2015.

[53] Wey Yang Teoh, Jason A. Scott, and Rose Amal. Progress in heterogeneous photocatalysis: From classical radical chemistry to engineering nanomaterials and solar reactors. Journal of Physical Chemistry Letters, 3(5):629-639, 2012.

[54] Shigeru Ikeda, Noboru Sugiyama, Bonamali Pal, Giuseppe Marcí, Leonardo Palmisano, Hidenori Noguchi, Kohei Uosaki, and Bunsho Ohtani. Photocatalytic activity of transition-metal-loaded titanium(IV) oxide powders suspended in aqueous solutions: Correlation with electron-hole recombination kinetics. Physical Chemistry Chemical Physics, 3(2):267-273, 2001.

[55] S. Malato, P. Fernández-Ibáñez, M.I. Maldonado, J. Blanco, and W. Gernjak. Decontamination and disinfection of water by solar photocatalysis: Recent overview and trends. Catalysis Today, 147(1):1-59, sep 2009.

[56] Andrea Testino, Ignazio Renato Bellobono, Vincenzo Buscaglia, Carmen Canevali, Massimiliano D'Arienzo, Stefano Polizzi, Roberto Scotti, and Franca Morazzoni. Optimizing the Photocatalytic Properties of Hydrothermal TiO2 by the Control of Phase Composition and Particle Morphology. A Systematic Approach. Journal of the American Chemical Society, 129(12):3564-3575, 2007. 
[57] Chen-Chi Wang, Zhibo Zhang, and Jackie Y. Ying. Photocatalytic decomposition of halogenated organics over nanocrystalline titania. Nanostructured Materials, 9(1-8):583-586, jan 1997.

[58] Zhibo Zhang, Chen-Chi Wang, Rama Zakaria, and Jackie Y Ying. Role of Particle Size in Nanocrystalline TiO2-Based Photocatalysts. The Journal of Physical Chemistry B, 102(52):10871-10878, 1998.

[59] Jean-Marie Herrmann. Heterogeneous photocatalysis: fundamentals and applications to the removal of various types of aqueous pollutants. Catalysis Today, 53(1):115-129, oct 1999.

[60] Michael R. Hoffmann, Scot T. Martin, Wonyong Choi, and Detlef W. Bahnemann. Environmental Applications of Semiconductor Photocatalysis. Chemical Reviews, 95(1):69-96, jan 1995.

[61] David F. Ollis. Photocatalytic purification and remediation of contaminated air and water. Comptes Rendus de l'Académie des Sciences Series IIC - Chemistry, 3(6):405-411, nov 2000.

[62] C Turchi. Photocatalytic degradation of organic water contaminants: Mechanisms involving hydroxyl radical attack. Journal of Catalysis, 122(1):178-192, 1990.

[63] Mahsa Motegh, Jiajun Cen, Peter W. Appel, J. Ruud van Ommen, and Michiel T. Kreutzer. Photocatalytic-reactor efficiencies and simplified expressions to assess their relevance in kinetic experiments. Chemical Engineering Journal, 207-208(0):607-615, oct 2012.

[64] Kristof Demeestere, Alex De Visscher, Jo Dewulf, Maarten Van Leeuwen, and Herman Van Langenhove. A new kinetic model for titanium dioxide mediated heterogeneous photocatalytic degradation of trichloroethylene in gas-phase. Applied Catalysis B: Environmental, 54(4):261-274, dec 2004.

[65] Morten G. Nielsen, Su-Il In, Peter C.K. Vesborg, Thomas Pedersen, Klaus P. Almtoft, Inge H. Andersen, Ole Hansen, and Ib Chorkendorff. A generic model for photocatalytic activity as a function of catalyst thickness. Journal of Catalysis, 289(0):62-72, may 2012.

[66] C. Colbeau-Justin and M. A. Valenzuela. Time-resolved microwave conductivity (TRMC) a useful characterization tool for charge carrier 
transfer in photocatalysis: A short review. Revista Mexicana de Fisica, 59(3):191-200, 2013.

[67] Aura Visan, Damon Rafieian, Wojciech Ogieglo, and Rob G H Lammertink. Modeling intrinsic kinetics in immobilized photocatalytic microreactors. Applied Catalysis B: Environmental, 150-151:93-100, 2014.

[68] Erik S Thiele and Roger H French. Light-Scattering Properties of Representative, Morphological Rutile Titania Particles Studied Using a FiniteElement Method. J. Am. Ceram. Soc., 81(3):469-479, 1998.

[69] Jorge Ripoll. Derivation of the scalar radiative transfer equation from energy conservation of Maxwell's equations in the far field. Journal of the Optical Society of America. A, Optics, image science, and vision, 28(8):1765-1775, 2011.

[70] Rodolfo J. Brandi, Orlando M. Alfano, and Alberto E. Cassano. Modeling of radiation absorption in a flat plate photocatalytic reactor. Chemical Engineering Science, 51(11):3169-3174, jun 1996.

[71] María I Cabrera, Orlando M Alfano, and Alberto E Cassano. Absorption and Scattering Coefficients of Titanium Dioxide Particulate Suspensions in Water. Journal of Physical Chemistry, 100(51):20043-20050, 1996.

[72] Alberto E. Cassano and Orlando M. Alfano. Reaction engineering of suspended solid heterogeneous photocatalytic reactors. Catalysis Today, 58(2-3):167-197, may 2000.

[73] G. Spadoni, E. Bandini, and F. Santarelli. Scattering effects in photosensitized reactions. Chemical Engineering Science, 33(4):517-524, 1978.

[74] J.L. Domínguez-Arvizu, J.A. Jiménez-Miramontes, J.M. SalinasGutiérrez, M.J. Meléndez-Zaragoza, A. López-Ortiz, and V. CollinsMartínez. Study of NiFe2O4 nanoparticles optical properties by a sixflux radiation model towards the photocatalytic hydrogen production. International Journal of Hydrogen Energy, pages 1-8, nov 2018.

[75] Miguel Angel Mueses, Fiderman Machuca-Martinez, Aracely HernándezRamirez, and Gianluca Li Puma. Effective radiation field model to scattering - Absorption applied in heterogeneous photocatalytic reactors. Chemical Engineering Journal, 279:442-451, nov 2015. 
[76] Sayra L. Orozco, Heidi I. Villafán-Vidales, and Camilo A. ArancibiaBulnes. Photon absorption in a hybrid slurry photocatalytic reactor: Assessment of differential approximations. AIChE Journal, 58(10):32563265 , oct 2012 .

[77] Gianluca Li Puma, Alberto Brucato, Gianluca Li Puma, and Alberto Brucato. Dimensionless analysis of slurry photocatalytic reactors using two-flux and six-flux radiation absorption-scattering models. Catalysis Today, 122(1-2):78-90, apr 2007.

[78] Alberto Brucato and Lucio Rizzuti. Simplified modeling of radiant fields in Heterogeneous photoreactors. 2. Limiting "two-flux" model for the case of reflectance greater than zero. Industrial \& Engineering Chemistry Research, 36(11):4748-4755, 1997.

[79] Gianluca Li Puma. Modeling of Thin-Film Slurry Photocatalytic Reactors Affected by Radiation Scattering. Environmental Science and Technology, 37(24):5783-5791, 2003.

[80] Mahsa Motegh, J. Ruud van Ommen, Peter W. Appel, Robert F. Mudde, and Michiel T. Kreutzer. Bubbles scatter light, yet that does not hurt the performance of bubbly slurry photocatalytic reactors. Chemical Engineering Science, 100(0):506-514, aug 2013.

[81] Andrea Turolla, Domenico Santoro, John R. de Bruyn, Ferdinando Crapulli, and Manuela Antonelli. Nanoparticle scattering characterization and mechanistic modelling of $\mathrm{UV}-\mathrm{TiO} 2$ photocatalytic reactors using computational fluid dynamics. Water Research, 88:117-126, jan 2016.

[82] Raúl Acosta-Herazo, Jesús Monterroza-Romero, Miguel Ángel Mueses, Fiderman Machuca-Martínez, and Gianluca Li Puma. Coupling the Six Flux Absorption-Scattering Model to the Henyey-Greenstein scattering phase function: Evaluation and optimization of radiation absorption in solar heterogeneous photoreactors. Chemical Engineering Journal, $302: 86-96$, oct 2016.

[83] María L. Satuf, Rodolfo J. Brandi, Alberto E. Cassano, and Orlando M. Alfano. Experimental Method to Evaluate the Optical Properties of Aqueous Titanium Dioxide Suspensions. Industrial \& Engineering Chemistry Research, 44(17):6643-6649, aug 2005. 
[84] J. Moreira, B. Serrano, A. Ortiz, and H. de Lasa. TiO2 absorption and scattering coefficients using Monte Carlo method and macroscopic balances in a photo-CREC unit. Chemical Engineering Science, 66(23):5813-5821, dec 2011.

[85] P.J. Valades-Pelayo, J. Moreira, B. Serrano, and H. de Lasa. Boundary conditions and phase functions in a Photo-CREC Water-II reactor radiation field. Chemical Engineering Science, 107:123-136, apr 2014.

[86] Craig E Bohren and Donald R Huffman Wiley. Absorption and Scattering of Light by Small Particles, volume 98. Wiley-VCH Verlag GmbH, Weinheim, Germany, apr 1998.

[87] Michael P. Diebold. Application of Light Scattering to Coatings. Springer International Publishing, Cham, 2014.

[88] H. C. van de Hulst. Light scattering by small particles. By H. C. van de Hulst. New York (John Wiley and Sons), London (Chapman and Hall), 1957. Pp. xiii, 470; 103 Figs.; 46 Tables. 96s. Quarterly Journal of the Royal Meteorological Society, 84(360):198-199, apr 1958.

[89] Damon Rafieian, Rick T. Driessen, Wojciech Ogieglo, and Rob G.H. Lammertink. Intrinsic Photocatalytic Assessment of Reactively Sputtered TiO 2 Films. ACS Applied Materials \& Interfaces, 7(16):87278732, apr 2015.

[90] John Butt. Reaction Kinetics and Reactor Design, 1999.

[91] Carberry. Chemical Reaction and Reactor Engineering. In Chemical Reaction and Reactor Engineering, pages 973-1054. CRC Press, 1987.

[92] Kanheya Mehrotra, Gregory S Yablonsky, and Ajay K Ray. Macro kinetic studies for photocatalytic degradation of benzoic acid in immobilized systems. Chemosphere, 60(10):1427-1436, 2005.

[93] Aymen Amine Assadi, Abdelkrim Bouzaza, and Dominique Wolbert. Photocatalytic oxidation of trimethylamine and isovaleraldehyde in an annular reactor: Influence of the mass transfer and the relative humidity. Journal of Photochemistry and Photobiology A: Chemistry, 236(0):6169, 2012. 
[94] Nu Ri Jung. Clarifying the legal ambiguity in article 2.2.2(III) of the anti-dumping agreement: A proposed set of interpretative guidelines for "Any other reasonable method". Asian Journal of WTO and International Health Law and Policy, 11(2):369-394, aug 2016.

[95] Octave Levenspiel. Chemical Reaction Engineering. 1999.

[96] Y. Nur, J.R. Lead, and M. Baalousha. Evaluation of charge and agglomeration behavior of $\mathrm{TiO} 2$ nanoparticles in ecotoxicological media. Science of The Total Environment, 535:45-53, dec 2015.

[97] Sang Bum Kim and Sung Chang Hong. Kinetic study for photocatalytic degradation of volatile organic compounds in air using thin film $\mathrm{TiO} 2$ photocatalyst. Applied Catalysis B: Environmental, 35(4):305-315, 2002.

[98] Kalithasan Natarajan, Thillai Sivakumar Natarajan, H C Bajaj, and Rajesh J Tayade. Photocatalytic reactor based on UV-LED/TiO2 coated quartz tube for degradation of dyes. Chemical Engineering Journal, 178(0):40-49, 2011.

[99] Hai Yang, Guiying Li, Taicheng An, Yanpeng Gao, and Jiamo Fu. Photocatalytic degradation kinetics and mechanism of environmental pharmaceuticals in aqueous suspension of $\mathrm{TiO} 2$ : A case of sulfa drugs. Catalysis Today, 153(3-4):200-207, 2010.

[100] G Plantard, F Correia, and V Goetz. Kinetic and efficiency of TiO2coated on foam or tissue and $\mathrm{TiO} 2$-suspension in a photocatalytic reactor applied to the degradation of the 2,4-dichlorophenol. Journal of Photochemistry and Photobiology A: Chemistry, 222(1):111-116, 2011.

[101] F Shahrezaei, Yadollah Mansouri, Ali Akbar Lorestani Zinatizadeh, and Aazam Akhbari. Process modeling and kinetic evaluation of petroleum refinery wastewater treatment in a photocatalytic reactor using $\mathrm{TiO} 2$ nanoparticles. Powder Technology, 221(0):203-212, 2012.

[102] Gordon S Shephard, Sonja Stockenström, David de Villiers, Willem J Engelbrecht, and Gabriël F S Wessels. Degradation of microcystin toxins in a falling film photocatalytic reactor with immobilized titanium dioxide catalyst. Water Research, 36(1):140-146, 2002. 
[103] Rongchang Wang, Dianjun Ren, Siqing Xia, Yalei Zhang, and Jianfu Zhao. Photocatalytic degradation of Bisphenol A (BPA) using immobilized $\mathrm{TiO} 2$ and $\{\mathrm{UV}\}$ illumination in a horizontal circulating bed photocatalytic reactor (HCBPR). Journal of Hazardous Materials, 169(13):926-932, 2009.

[104] Wen-Yu Wang, Agus Irawan, and Young Ku. Photocatalytic degradation of Acid Red 4 using a titanium dioxide membrane supported on a porous ceramic tube. Water Research, 42(19):4725-4732, 2008.

[105] Zhonghai Zhang, Hongjun Wu, Yuan Yuan, Yanju Fang, and Litong Jin. Development of a novel capillary array photocatalytic reactor and application for degradation of azo dye. Chemical Engineering Journal, 184(0):9-15, 2012.

[106] Saber Ahmed, M G Rasul, R Brown, and M A Hashib. Influence of parameters on the heterogeneous photocatalytic degradation of pesticides and phenolic contaminants in wastewater: A short review. Journal of Environmental Management, 92(3):311-330, 2011.

[107] Meng Nan Chong, Bo Jin, Christopher W K Chow, and Chris Saint. Recent developments in photocatalytic water treatment technology: A review. Water Research, 44(10):2997-3027, 2010.

[108] María L Satuf, María J Pierrestegui, Lorena Rossini, Rodolfo J Brandi, and Orlando M Alfano. Kinetic modeling of azo dyes photocatalytic degradation in aqueous $\mathrm{TiO} 2$ suspensions. Toxicity and biodegradability evaluation. Catalysis Today, 161(1):121-126, 2011.

[109] María L Satuf, Rodolfo J Brandi, Alberto E Cassano, and Orlando M Alfano. Scaling-up of slurry reactors for the photocatalytic degradation of 4-chlorophenol. Catalysis Today, 129(1-2):110-117, 2007.

[110] Claudio Passalia, Orlando M Alfano, and Rodolfo J Brandi. A methodology for modeling photocatalytic reactors for indoor pollution control using previously estimated kinetic parameters. Journal of Hazardous Materials, 211-212(0):357-365, 2012.

[111] Javier Marugan, Rafael van Grieken, Cristina Pablos, M Lucila Satuf, Alberto E Cassano, and Orlando M Alfano. Modeling of a bench-scale photocatalytic reactor for water disinfection from laboratory-scale kinetic data. Chemical Engineering Journal, 224(0):39-45, 2013. 
[112] Javier Marugan, Rafael van Grieken, Alberto E Cassano, and Orlando M Alfano. Scaling-up of slurry reactors for the photocatalytic oxidation of cyanide with $\mathrm{TiO} 2$ and silica-supported $\mathrm{TiO} 2$ suspensions. Catalysis Today, 144(1-2):87-93, 2009.

[113] Javier Marugan, Rafael van Grieken, Alberto E Cassano, and Orlando M Alfano. Intrinsic kinetic modeling with explicit radiation absorption effects of the photocatalytic oxidation of cyanide with $\mathrm{TiO} 2$ and silicasupported TiO2 suspensions. Applied Catalysis B: Environmental, 85(12):48-60, 2008.

[114] N Doucet, O Zahraa, and M Bouchy. Kinetics of the photocatalytic degradation of benzene. Catalysis Today, 122(1-2):168-177, 2007.

[115] J. Esteban Duran, Madjid Mohseni, and Fariborz Taghipour. Modeling of annular reactors with surface reaction using computational fluid dynamics (CFD). Chemical Engineering Science, 65(3):1201-1211, feb 2010 .

[116] Madjid Mohseni and Fariborz Taghipour. Experimental and $\{\mathrm{CFD}\}$ analysis of photocatalytic gas phase vinyl chloride (VC) oxidation. Chemical Engineering Science, 59(7):1601-1609, 2004.

[117] Jesus Moreira, Benito Serrano, Aaron Ortiz, and Hugo de Lasa. A unified kinetic model for phenol photocatalytic degradation over $\mathrm{TiO} 2$ photocatalysts. Chemical Engineering Science, 78(0):186-203, 2012.

[118] Miguel Angel Mueses, Fiderman Machuca-Martinez, and Gianluca Li Puma. Effective quantum yield and reaction rate model for evaluation of photocatalytic degradation of water contaminants in heterogeneous pilot-scale solar photoreactors. Chemical Engineering Journal, 215-216(0):937-947, 2013.

[119] Roberto L Pozzo, Rodolfo J Brandi, Alberto E Cassano, and Miguel A Baltanás. Photocatalytic oxidation of oxalic acid in dilute aqueous solution, in a fully illuminated fluidized bed reactor. Chemical Engineering Science, 65(4):1345-1353, 2010.

[120] María de los Milagros Ballari, Rodolfo Brandi, Orlando Alfano, and Alberto Cassano. Mass transfer limitations in photocatalytic reactors 
employing titanium dioxide suspensions: II. External and internal particle constrains for the reaction. Chemical Engineering Journal, 136(2$3): 242-255,2008$.

[121] María de los Milagros Ballari, Rodolfo Brandi, Orlando Alfano, Alberto Cassano, María de los Milagros Ballari, Rodolfo Brandi, Orlando Alfano, and Alberto Cassano. Mass transfer limitations in photocatalytic reactors employing titanium dioxide suspensions. Chemical Engineering Journal, 136(1):50-65, feb 2008.

[122] María de los Milagros Ballari, Orlando M. Alfano, and Alberto E. Cassano. Mass transfer limitations in slurry photocatalytic reactors: Experimental validation. Chemical Engineering Science, 65(17):4931-4942, sep 2010 .

[123] C. a. Arancibia-Bulnes and S. a. Cuevas. Modeling of the radiation field in a parabolic trough solar photocatalytic reactor. Solar Energy, 76(5):615-622, 2004.

[124] Rodolfo J. Brandi, Miguel a. Citroni, Orlando M. Alfano, and Alberto E. Cassano. Absolute quantum yields in photocatalytic slurry reactors. Chemical Engineering Science, 58(3-6):979-985, feb 2003.

[125] Gustavo E. Imoberdorf, Fariborz Taghipour, Mehrdad Keshmiri, and Madjid Mohseni. Predictive radiation field modeling for fluidized bed photocatalytic reactors. Chemical Engineering Science, 63(16):42284238, 2008.

[126] Gianluca Li Puma and Alberto Brucato. Dimensionless analysis of slurry photocatalytic reactors using two-flux and six-flux radiation absorption - scattering models. Catalysis Today, 122:78-90, 2007.

[127] Franjo Jovic, Vanja Kosar, Vesna Tomasic, and Zoran Gomzi. Nonideal flow in an annular photocatalytic reactor. Chemical Engineering Research and Design, 90(9):1297-1306, 2012.

[128] Gianluca Li Puma and Po Lock Yue. Modelling and design of thin-film slurry photocatalytic reactors for water purification. Chemical Engineering Science, 58(11):2269-2281, 2003.

[129] Gianluca Li Puma and Po Lock Yue. A novel fountain photocatalytic reactor: model development and experimental validation. Chemical Engineering Science, 56(8):2733-2744, 2001. 
[130] Gianluca Li Puma and Po Lock Yue. A laminar falling film slurry photocatalytic reactor. Part I - model development. Chemical Engineering Science, 53(16):2993-3006, 1998.

[131] Gustavo E Imoberdorf, Horacio A Irazoqui, Orlando M Alfano, and Alberto E Cassano. Scaling-up from first principles of a photocatalytic reactor for air pollution remediation. Chemical Engineering Science, 62(3):793-804, 2007.

[132] Gustavo E Imoberdorf, Alberto E Cassano, Horacio A Irazoqui, and Orlando M Alfano. Optimal design and modeling of annular photocatalytic wall reactors. Catalysis Today, 129(1-2):118-126, 2007.

[133] Vesna Tomasic, Franjo Jovic, and Zoran Gomzi. Photocatalytic oxidation of toluene in the gas phase: Modelling an annular photocatalytic reactor. Catalysis Today, 137(2-4):350-356, 2008.

[134] Ignasi Salvado-Estivill, Alberto Brucato, and Gianluca Li Puma. TwoDimensional Modeling of a Flat-Plate Photocatalytic Reactor for Oxidation of Indoor Air Pollutants. Industrial \& Engineering Chemistry Research, 46(23):7489-7496, 2007.

[135] Zimeng Wang, Jing Liu, Yuancan Dai, Weiyang Dong, Shicheng Zhang, and Jianmin Chen. CFD modeling of a UV-LED photocatalytic odor abatement process in a continuous reactor. Journal of Hazardous Materials, 215-216(0):25-31, 2012.

[136] M F J Dijkstra, H J Panneman, J G M Winkelman, J J Kelly, and A.A.C.M. Beenackers. Modeling the photocatalytic degradation of formic acid in a reactor with immobilized catalyst. Chemical Engineering Science, 57(22-23):4895-4907, 2002.

[137] Aymen Amine Assadi, Jordi Palau, Abdelkrim Bouzaza, and Dominique Wolbert. Modeling of a continuous photocatalytic reactor for isovaleraldehyde oxidation: Effect of different operating parameters and chemical degradation pathway. Chemical Engineering Research and Design, 91(7):1307-1316, 2013.

[138] Benoit Boulinguiez, Abdelkrim Bouzaza, Smail Merabet, and Dominique Wolbert. Photocatalytic degradation of ammonia and butyric acid in plug-flow reactor: Degradation kinetic modeling with contribution of 
mass transfer. Journal of Photochemistry and Photobiology A: Chemistry, 200(2-3):254-261, 2008.

[139] V Goetz, J P Cambon, D Sacco, and G Plantard. Modeling aqueous heterogeneous photocatalytic degradation of organic pollutants with immobilized TiO2. Chemical Engineering and Processing: Process Intensification, 48(1):532-537, 2009.

[140] R Gorges. Photocatalysis in microreactors. Journal of Photochemistry and Photobiology A: Chemistry, 167(2-3):95-99, oct 2004.

[141] Y MATSUSHITA, N OHBA, S KUMADA, K SAKEDA, T SUZUKI, and T ICHIMURA. Photocatalytic reactions in microreactors. Chemical Engineering Journal, 135(SUPPL. 1):S303-S308, jan 2008.

[142] Lei Lei, Ning Wang, X M Zhang, Qidong Tai, Din Ping Tsai, and Helen L W Chan. Optofluidic planar reactors for photocatalytic water treatment using solar energy. Biomicrofluidics, 4(4):43004, 2010.

[143] Zhaoxu Meng, Xu Zhang, and Jianhua Qin. A high efficiency microfluidic-based photocatalytic microreactor using electrospun nanofibrous TiO2 as a photocatalyst. Nanoscale, 5(11):4687-4690, 2013.

[144] Henrik Lindstrom, Robert Wootton, and Alexander Iles. High surface area titania photocatalytic microfluidic reactors. AIChE Journal, 53(3):695-702, 2007.

[145] Guillaume Charles, Thibault Roques-Carmes, Nidhal Becheikh, Laurent Falk, Jean-Marc Commenge, and Serge Corbel. Determination of kinetic constants of a photocatalytic reaction in micro-channel reactors in the presence of mass-transfer limitation and axial dispersion. Journal of Photochemistry and Photobiology A: Chemistry, 223(2-3):202-211, 2011.

[146] D A G Bruggeman. Berechnung verschiedener physikalischer Konstanten von heterogenen Substanzen. I. Dielektrizitatskonstanten und Leitfahigkeiten der Mischkarper aus isotropen Substanzen. Annalen der Physik, 416:636-664, 1935.

[147] Blaine Johs and Jeffrey S Hale. Dielectric function representation by B-splines. physica status solidi (a), 205:715-719, 2008. 
[148] Jussi Kasanen, Janne Salstela, Mika Suvanto, and Tuula T Pakkanen. Photocatalytic degradation of methylene blue in water solution by multilayer TiO2 coating on HDPE. Applied Surface Science, 258:1738-1743, 2011.

[149] Bozhidar I Stefanov, Nina V Kaneva, Gianluca Li Puma, and Ceco D Dushkin. Novel integrated reactor for evaluation of activity of supported photocatalytic thin films: Case of methylene blue degradation on $\mathrm{TiO} 2$ and nickel modified TiO2 under $\{\mathrm{UV}\}$ and visible light. Colloids and Surfaces A: Physicochemical and Engineering Aspects, 382(1-3):219-225, 2011.

[150] Shiying Zhang, Zhenhua Chen, Yunlong Li, Qun Wang, and Long Wan. Photocatalytic degradation of methylene blue in a sparged tube reactor with $\mathrm{TiO} 2$ fibers prepared by a properly two-step method. Catalysis Communications, 9:1178-1183, 2008.

[151] Hang Xu, Mei Li, and Zhang Jun. Preparation, characterization, and photocatalytic studies on anatase nano-TiO2 at internal air lift circulating photocatalytic reactor. Materials Research Bulletin, 48:3144-3148, 2013.

[152] Yajun Wang, Jie Lin, Ruilong Zong, Jun He, and Yongfa Zhu. Enhanced photoelectric catalytic degradation of methylene blue via $\mathrm{TiO} 2$ nanotube arrays hybridized with graphite-like carbon. Journal of Molecular Catalysis A: Chemical, 349:13-19, 2011.

[153] L Rizzo, J Koch, V Belgiorno, and M A Anderson. Removal of methylene blue in a photocatalytic reactor using polymethylmethacrylate supported TiO2 nanofilm. Desalination, 211:1-9, 2007.

[154] Hinda Lachheb, Eric Puzenat, Ammar Houas, Mohamed Ksibi, Elimame Elaloui, Chantal Guillard, and Jean-Marie Herrmann. Photocatalytic degradation of various types of dyes (Alizarin S, Crocein Orange G, Methyl Red, Congo Red, Methylene Blue) in water by UV-irradiated titania. Applied Catalysis B: Environmental, 39:75-90, 2002.

[155] A R Khataee, M Fathinia, and S W Joo. Simultaneous monitoring of photocatalysis of three pharmaceuticals by immobilized TiO2 nanoparticles: Chemometric assessment, intermediates identification and ecotoxicological evaluation. Spectrochimica Acta Part A: Molecular and Biomolecular Spectroscopy, 112:33-45, 2013. 
[156] Kyung-Jun Hwang, Jae-Wook Lee, Wang-Geun Shim, Hee Dong Jang, Se-Il Lee, and Seung-Joon Yoo. Adsorption and photocatalysis of nanocrystalline $\mathrm{TiO} 2$ particles prepared by sol-gel method for methylene blue degradation. Advanced Powder Technology, 23:414-418, 2012.

[157] Jihyun Choi, Hongshin Lee, Yeoseon Choi, Soonhyun Kim, Seokheon Lee, Seunghak Lee, Wonyong Choi, and Jaesang Lee. Heterogeneous photocatalytic treatment of pharmaceutical micropollutants: Effects of wastewater effluent matrix and catalyst modifications. Applied Catalysis B: Environmental, 147:8-16, 2014.

[158] Halil Can Aran. Porous ceramic and metallic microreactors : tuning interfaces for multiphase processes. PhD thesis, University of Twente, Enschede, The Netherlands, nov 2011.

[159] Jean-Marie Herrmann. Photocatalysis fundamentals revisited to avoid several misconceptions. Applied Catalysis B: Environmental, 99:461-468, 2010 .

[160] G. Camera-Roda and F. Santarelli. Optimization of the thickness of a photocatalytic film on the basis of the effectiveness factor. Catalysis Today, 129(1-2):161-168, nov 2007.

[161] George W Huber, Sara Iborra, and Avelino Corma. Synthesis of Transportation Fuels from Biomass: Chemistry, Catalysts, and Engineering. Chemical Reviews, 106(9):4044-4098, 2006.

[162] Ayhan Demirbaş. Biomass resource facilities and biomass conversion processing for fuels and chemicals. Energy Conversion and Management, 42(11):1357-1378, 2001.

[163] J. S. Luterbacher, D. Martin Alonso, and J. A. Dumesic. Targeted chemical upgrading of lignocellulosic biomass to platform molecules. Green Chem., 16(12):4816-4838, oct 2014.

[164] E de Jong, M A Dam, L Sipos, and G.-J. M Gruter. Furandicarboxylic Acid (FDCA), A Versatile Building Block for a Very Interesting Class of Polyesters, chapter 1, pages 1-13.

[165] P N R Vennestrom, C M Osmundsen, C H Christensen, and Esben Taarning. Beyond petrochemicals: the renewable chemicals industry. Angewandte Chemie (International ed. in English), 50(45):10502-10509, nov 2011. 
[166] Xian-Long Du, Lin He, She Zhao, Yong-Mei Liu, Yong Cao, He-Yong He, and Kang-Nian Fan. Hydrogen-Independent Reductive Transformation of Carbohydrate Biomass into $\gamma$-Valerolactone and Pyrrolidone Derivatives with Supported Gold Catalysts. Angewandte Chemie International Edition, 50(34):7815-7819, 2011.

[167] Xing Tang, Huawei Chen, Lei Hu, Weiwei Hao, Yong Sun, Xianhai Zeng, Lu Lin, and Shijie Liu. Conversion of biomass to $\gamma$-valerolactone by catalytic transfer hydrogenation of ethyl levulinate over metal hydroxides. Applied Catalysis B: Environmental, 147:827-834, 2014.

[168] Michiel Dusselier, Mark Mascal, and Bert F. Sels. Top Chemical Opportunities from Carbohydrate Biomass: A Chemist's View of the Biorefinery. pages 1-40. 2014.

[169] Yi-Chun Lee, Ching-Tien Chen, Yu-Ting Chiu, and Kevin C.-W. Wu. An Effective Cellulose-to-Glucose-to-Fructose Conversion Sequence by Using Enzyme Immobilized Fe 3 O 4 -Loaded Mesoporous Silica Nanoparticles as Recyclable Biocatalysts. ChemCatChem, 5(8):21532157, aug 2013.

[170] Jitendra Kumar Saini, Reetu Saini, and Lakshmi Tewari. Lignocellulosic agriculture wastes as biomass feedstocks for second-generation bioethanol production: concepts and recent developments. 3 Biotech, 5(4):337-353, aug 2015.

[171] Masashi Hattori, Keigo Kamata, and Michikazu Hara. Photoassistphosphorylated $\mathrm{TiO} 2$ as a catalyst for direct formation of 5(hydroxymethyl)furfural from glucose. Phys. Chem. Chem. Phys., 19(5):3688-3693, 2017.

[172] Rodrigo Lopes De Souza, Hao Yu, Franck Rataboul, and Nadine Essayem. 5-Hydroxymethylfurfural (5-HMF) Production from Hexoses: Limits of Heterogeneous Catalysis in Hydrothermal Conditions and Potential of Concentrated Aqueous Organic Acids as Reactive Solvent System. Challenges, 3(2):212-232, 2012.

[173] Xian-Long Du, Qing-Yuan Bi, Yong-Mei Liu, Yong Cao, and KangNian Fan. Conversion of biomass-derived levulinate and formate esters into gamma-valerolactone over supported gold catalysts. ChemSusChem, 4(12):1838-1843, dec 2011. 
[174] Kai Yan and Aicheng Chen. Selective hydrogenation of furfural and levulinic acid to biofuels on the ecofriendly $\mathrm{Cu}-\mathrm{Fe}$ catalyst. Fuel, 115:101108, 2014.

[175] Mei Chia and James A. Dumesic. Liquid-phase catalytic transfer hydrogenation and cyclization of levulinic acid and its esters to $\gamma$-valerolactone over metal oxide catalysts. Chemical Communications, 47(44):12233, 2011.

[176] Masaru Watanabe, Yuichi Aizawa, Toru Iida, Ryo Nishimura, and Hiroshi Inomata. Catalytic glucose and fructose conversions with $\mathrm{TiO} 2$ and $\mathrm{ZrO} 2$ in water at $473 \mathrm{~K}$ : Relationship between reactivity and acidbase property determined by TPD measurement. Applied Catalysis A: General, 295(2):150-156, nov 2005.

[177] Kiyotaka Nakajima, Ryouhei Noma, Masaaki Kitano, and Michikazu Hara. Selective glucose transformation by titania as a heterogeneous Lewis acid catalyst. Journal of Molecular Catalysis A: Chemical, 388389:100-105, 2014.

[178] V V Ordomsky, V L Sushkevich, J C Schouten, J Van Der Schaaf, and $\mathrm{T}$ a. Nijhuis. Glucose dehydration to 5-hydroxymethylfurfural over phosphate catalysts. Journal of Catalysis, 300:37-46, 2013.

[179] Yomaira J. Pagán-Torres, Tianfu Wang, Jean Marcel R Gallo, Brent H. Shanks, and James a. Dumesic. Production of 5-hydroxymethylfurfural from glucose using a combination of lewis and brønsted acid catalysts in water in a biphasic reactor with an alkylphenol solvent. ACS Catalysis, 2(6):930-934, 2012.

[180] H. Zhao, J. E. Holladay, H. Brown, and Z. C. Zhang. Metal Chlorides in Ionic Liquid Solvents Convert Sugars to 5-Hydroxymethylfurfural. Science, 316(5831):1597-1600, jun 2007.

[181] B. G. Siqueira, M. A. P. Silva, and C. Moraes. SYNTHESIS OF HMF FROM GLUCOSE IN AQUEOUS MEDIUM USING NIOBIUM AND TITANIUM OXIDES. Brazilian Journal of Petroleum and Gas, 7(2):7182 , jun 2013.

[182] Samuel Marre, Jinyoung Baek, Jongnam Park, Moungi G. Bawendi, and Klavs F. Jensen. High-Pressure/High-Temperature Microreactors 
for Nanostructure Synthesis. Journal of the Association for Laboratory Automation, 14(6):367-373, dec 2009.

[183] Paolo Carniti, Antonella Gervasini, Serena Biella, and Aline Auroux. Niobic acid and niobium phosphate as highly acidic viable catalysts in aqueous medium: Fructose dehydration reaction. Catalysis Today, 118(3-4 SPEC. ISS.):373-378, 2006.

[184] E. W. Thiele. Relation between Catalytic Activity and Size of Particle. Industrial \& Engineering Chemistry, 31(7):916-920, jul 1939.

[185] N. Raveendran Shiju and Vadim V. Guliants. Recent developments in catalysis using nanostructured materials. Applied Catalysis A: General, 356(1):1-17, mar 2009.

[186] J. Anderson. Colloid Transport By Interfacial Forces. Annual Review of Fluid Mechanics, 21(1):61-99, jan 1989.

[187] J. P. Ebel, J. L. Anderson, and D. C. Prieve. Diffusiophoresis of Latex Particles in Electrolyte Gradients. Langmuir, 4(2):396-406, 1988.

[188] Menachem Elimelech and Charles R O'Melia. Effect of electrolyte type on the electrophoretic mobility of polystyrene latex colloids. Colloids and Surfaces, 44(C):165-178, 1990.

[189] Sangwoo Shin, Jesse T Ault, Jie Feng, Patrick B Warren, and Howard A Stone. Low-Cost Zeta Potentiometry Using Solute Gradients. Advanced Materials, 29(30):1-7, 2017.

[190] D. Florea, S. Musa, J. M. R. Huyghe, and H. M. Wyss. Long-range repulsion of colloids driven by ion exchange and diffusiophoresis. Proceedings of the National Academy of Sciences, 111(18):6554-6559, 2014.

[191] Timothy R. Kline, Walter F. Paxton, Yang Wang, Darrell Velegol, Thomas E. Mallouk, and Ayusman Sen. Catalytic micropumps: Microscopic convective fluid flow and pattern formation. Journal of the American Chemical Society, 127(49):17150-17151, 2005.

[192] A. Afshar Farniya, M. J. Esplandiu, D. Reguera, and A. Bachtold. Imaging the Proton Concentration and Mapping the Spatial Distribution of the Electric Field of Catalytic Micropumps. Physical Review Letters, 111(16):168301, oct 2013. 
[193] Ali Afshar Farniya, Maria J. Esplandiu, and Adrian Bachtold. Sequential tasks performed by catalytic pumps for colloidal crystallization. Langmuir, 30(39):11841-11845, 2014.

[194] Scott. M. Davidson, Rob G. H. Lammertink, and Ali Mani. A Predictive Model for Convective Flows Induced by Surface Reactivity Contrast. Physical Review Fluids, 3(5):053701, may 2017.

[195] Rune Barnkob, Christian J. Kähler, and Massimiliano Rossi. General defocusing particle tracking. Lab on a Chip, 15(17):3556-3560, 2015.

[196] Walter Hayduk and Harry Laudie. Prediction of diffusion coefficients for nonelectrolytes in dilute aqueous solutions. AIChE Journal, 20(3):611615, may 1974.

[197] V. Augugliaro, H. A.Hamed El Nazer, V. Loddo, A. Mele, G. Palmisano, L. Palmisano, and S. Yurdakal. Partial photocatalytic oxidation of glycerol in TiO2 water suspensions. Catalysis Today, 151(1-2):21-28, 2010.

[198] A. Fernández-Nieves, F. J. Nieves, and C. Richter. Point of zero charge estimation for a $\mathrm{TiO} 2 /$ water interface. In Trends in Colloid and Interface Science XII, pages 21-24. Steinkopff, Darmstadt, 1998.

[199] Brian J Kirby and Ernest F Hasselbrink. Zeta potential of microfluidic substrates: 2. Data for polymers. ELECTROPHORESIS, 25(2):203-213, jan 2004.

[200] Vishal Tandon, Sharath K. Bhagavatula, Wyatt C. Nelson, and Brian J. Kirby. Zeta potential and electroosmotic mobility in microfluidic devices fabricated from hydrophobic polymers: 1 . The origins of charge. Electrophoresis, 29(5):1092-1101, 2008.

[201] Necmettin Cevheri and Minami Yoda. Lift forces on colloidal particles in combined electroosmotic and poiseuille flow. Langmuir, 30(46):1377113780, 2014.

[202] Mingren Shen, Fangfu Ye, Rui Liu, Ke Chen, Mingcheng Yang, and Marisol Ripoll. Chemically driven fluid transport in long microchannels. Journal of Chemical Physics, 145(12), 2016.

[203] Mingcheng Yang and Marisol Ripoll. A self-propelled thermophoretic microgear. Soft Matter, 10(7):1006-1011, 2014. 
[204] David A. Gregory and Stephen J. Ebbens. Symmetrical Catalytically Active Colloids Collectively Induce Convective Flow. Langmuir, 34(14):4307-4313, 2018.

[205] N. Miložič, M. Lubej, U. Novak, P. Žnidaršič-Plazl, and I. Plazl. Evaluation of Diffusion Coefficient Determination using a Microfluidic Device. Chemical and Biochemical Engineering Quarterly Journal, 28(2):215223, apr 2014.

[206] Derek G Leaist. The effects of aggregation, counterion binding, and added sodium chloride on diffusion of aqueous methylene blue. Canadian Journal of Chemistry, 66(9):2452-2457, 1988.

[207] Chuanxi Yang, Wenping Dong, Guanwei Cui, Yingqiang Zhao, Xifeng Shi, Xinyuan Xia, Bo Tang, and Weiliang Wang. Highly efficient photocatalytic degradation of methylene blue by P2ABSA-modified TiO2nanocomposite due to the photosensitization synergetic effect of TiO2and P2ABSA. RSC Advances, 7(38):23699-23708, 2017.

[208] Philippe Leroy, Christophe Tournassat, and Mohamed Bizi. Influence of surface conductivity on the apparent zeta potential of TiO2 nanoparticles. Journal of Colloid and Interface Science, 356(2):442-453, apr 2011.

[209] J Welty, C E Wicks, G L Rorrer, and R E Wilson. Fundamentals of Momentum, Heat and Mass Transfer. Wiley, 2007.

[210] Ran Niu, Denis Botin, Julian Weber, Alexander Reinmüller, and Thomas Palberg. Assembly and Speed in Ion-Exchange-Based Modular Phoretic Microswimmers. Langmuir, 33(14):3450-3457, 2017.

[211] Xi Zhao, Henri Palacci, Vinita Yadav, Michelle M Spiering, Michael K Gilson, Peter J Butler, Henry Hess, Stephen J Benkovic, and Ayusman Sen. Substrate-driven chemotactic assembly in an enzyme cascade. $\mathrm{Na}$ ture Chemistry, 10:311, dec 2017.

[212] Samudra Sengupta, Krishna K Dey, Hari S Muddana, Tristan Tabouillot, Michael E Ibele, Peter J Butler, and Ayusman Sen. Enzyme molecules as nanomotors. Journal of the American Chemical Society, 135(4):1406-1414, 2013. 
[213] Krishna K. Dey, Xi Zhao, Benjamin M. Tansi, Wilfredo J. Méndez-Ortiz, Ubaldo M. Córdova-Figueroa, Ramin Golestanian, and Ayusman Sen. Micromotors Powered by Enzyme Catalysis. Nano Letters, 15(12):8311$8315,2015$.

[214] Hua Yu, Kyubong Jo, Kristy L. Kounovsky, Juan J De Pablo, and David C. Schwartz. Molecular Propulsion: Chemical Sensing and Chemotaxis of DNA Driven by RNA Polymerase. Journal of the American Chemical Society, 131(16):5722-5723, apr 2009.

[215] Ammar Houas, Hinda Lachheb, Mohamed Ksibi, Elimame Elaloui, Chantal Guillard, and Jean Marie Herrmann. Photocatalytic degradation pathway of methylene blue in water. Applied Catalysis B: Environmental, 31(2):145-157, 2001.

[216] Monica Brivio, Roel H. Fokkens, Willem Verboom, David N. Reinhoudt, Niels R. Tas, Martijn Goedbloed, and Albert van den Berg. Integrated Microfluidic System Enabling (Bio)chemical Reactions with On-Line MALDI-TOF Mass Spectrometry. Analytical Chemistry, 74(16):39723976, aug 2002.

[217] Kevin Paul Nichols and J. G. E. Gardeniers. A Digital Microfluidic System for the Investigation of Pre-Steady-State Enzyme Kinetics Using Rapid Quenching with MALDI-TOF Mass Spectrometry. Analytical Chemistry, 79(22):8699-8704, nov 2007.

[218] A. Susarrey-Arce, R.M. Tiggelaar, M. Morassutto, J. Geerlings, R.G.P. Sanders, B. Geerdink, S. Schlautmann, L. Lefferts, A. van Houselt, and J.G.E. Gardeniers. A new ATR-IR microreactor to study electric fielddriven processes. Sensors and Actuators B: Chemical, 220:13-21, dec 2015.

[219] A. Susarrey-Arce, R. M. Tiggelaar, J. G. E. Gardeniers, A. van Houselt, and L. Lefferts. CO Adsorption on Pt Nanoparticles in Low E-Fields Studied by ATR-IR Spectroscopy in a Microreactor. The Journal of Physical Chemistry C, 119(44):24887-24894, nov 2015.

[220] E Vereshchagina, O Bliznyuk, R M Tiggelaar, K Altena-Schildkamp, and J G E Gardeniers. Local deposition and patterning of catalytic thin films in microsystems. Journal of Micromechanics and Microengineering, 22(4):045023, apr 2012. 
[221] Johan E ten Elshof, Sajid U Khan, and Ole F. Göbel. Micrometer and nanometer-scale parallel patterning of ceramic and organic-inorganic hybrid materials. Journal of the European Ceramic Society, 30(7):15551577, may 2010.

[222] Martin Heule, Urs P. Schönholzer, and Ludwig J. Gauckler. Patterning colloidal suspensions by selective wetting of microcontact-printed surfaces. Journal of the European Ceramic Society, 24(9):2733-2739, 2004. 